\title{
Laminated connections under tensile load at different temperatures and strain rates
}

Dr. Ing. Manuel Santarsiero (corresponding author)

1.

Eckersley O'Callaghan Engineers (EOC)

London, United Kingdom

2.

Steel Structures Laboratory (ICOM)

School of Architecture, Civil and Environmental Engineering (ENAC)

École Polytechnique Fédérale de Lausanne (EPFL)

Lausanne, Switzerland

manuel.santarsiero@gmail.com

$+447944967052$

$+41786282420$

\section{Dr. ir. Christian Louter}

Chair of Structural Design

Department of Architectural Engineering and Technology (AE+T)

Faculty of Architecture and the Built Environment (A+BE)

Delft University of Technology (TU Delft)

Delft, The Netherlands

Christian.Louter@TUDelft.nl

$+31628241871$

\section{Prof. Dr. Alain Nussbaumer}

Steel Structures Laboratory (ICOM)

School of Architecture, Civil and Environmental Engineering (ENAC)

École Polytechnique Fédérale de Lausanne (EPFL)

Lausanne, Switzerland

alain.nussbaumer@epfl.ch

+41216932425 


\begin{abstract}
In the last years, a novel typology of adhesive connections for structural glass application has emerged, known as laminated adhesive connections, which makes use of the transparent ionomer SentryGlas ${ }^{\circledR}(\mathrm{SG})$ from Kuraray and the Transparent Structural Silicon Adhesive (TSSA) from Dow Corning. Despite being used in several projects, limited information is available in literature on their mechanical behaviour and on the effects of strain rate and temperature. In this work the behaviour of laminated connections under tensile loading is studied by means of experimental, analytical and numerical analyses. The experimental investigations show that temperature and strain rate variations have important effects on the mechanical response of the connections. Two main interesting phenomena are also observed: the whitening phenomenon in TSSA and the development of bubble within the SG adhesive. The analytical studies of the stress state show that confinement state of the adhesive induces a non-uniform three-dimensional stress distribution in the adhesive with a dominant hydrostatic component of the stress tensor, which is observed to be in agreement with the experimental results. Three-dimensional finite numerical analyses show that the stress field deviates from the uniform distribution with a large gradient of hydrostatic and deviatoric stresses over the adhesive area. The output of the finite numerical model are then compared with the observations of the experimental campaigns. Herein, the full set of numerical results is synthetized by the definition of socalled stress factors. The latter allow to derive the three-dimensional stress state in the adhesive at different temperatures and to compute the stress peak in the non-linear stress field distribution. Finally, prediction models are proposed for the tensile resistance of TSSA and SG laminated connections. A logarithmic law is proposed for the strain rate effects for both TSSA and SG connections. Linear and inverse hyperbolic-tangent-based laws are instead proposed for the TSSA and SG temperature effects, respectively.
\end{abstract}

\title{
Keywords
}

Laminated adhesive connections, structural glass, experimental investigation, numerical modeling, tensile loading, strain rate effect, temperature effect 


\section{Introduction}

The demand for architectural transparency has drastically increased in the last decades. This trend has inevitably promoted the use of glass in buildings and constructions. Due to the fragile nature of this material, connections between structural glass components represent one of the main critical aspects of glass engineering. This is because glass cannot plastically redistribute the stress peaks occurring where forces are transferred between components. In comparison to bolted connections, adhesive connections are characterized by the following advantages: (i) the transfer of forces is distributed over the full bonded area thus avoiding contact stress intensification occurring in bolted connection, (ii) the drilling process and the subsequently reduction of glass strength at the bore hole edge of bolted connections is avoided, (iii) the architectural flushness is enhanced because the metal parts do not go through the glass, (iv) thermal bridges and thermal losses are reduced also because the metal part does not go through the entire glass thickness, (v) the residual stress field distribution of the tempering is unaltered at the connection, and (vi) gas losses occurring in IGU bolted panels are reduced since the glass is not drilled. Because of these aspects, the use of adhesive connections in structural applications has been considered very promising. Indeed, several research institutes have been performing investigations on adhesive connections for structural glass applications [1]-[12].

In the last years, a novel typology of adhesive connections has emerged, known as laminated adhesive connections. The main characteristic of laminated connections is that they make use of the same production process of laminated glass components. In addition, they exhibit high mechanical performances and full transparency. In laminated connections, a solid foil of transparent adhesive material is placed between a metal connector and glass panel. Metal, adhesive and glass are then typically placed in a vacuum bag and subjected to a standard autoclave process commonly applied for the production of laminated glass components. The lamination process is performed by simultaneous application of pressure and heat by means of an autoclave. At the end of the lamination process the result is a glass component where the metal part is fully bonded to the glass plate by means of the laminated transparent adhesive.

Laminated connections have been used in several projects such as Apple Retail stores worldwide. In these projects, laminated connections are used to realize the structural joints between the glass components such as façade panel, glass stairs, beams and columns. One of the most iconic examples is represented by the Apple store in New York (U.S.), on the $5^{\text {th }}$ avenue [13]. The main external structure is composed by $10 \mathrm{~m} \times 10 \mathrm{~m}$ glass portals that constitute a fully transparent glass cube structure (see Figure 1 (a)). The connections between structural glass elements (e.g. beam-to-beam connections (Figure 1 (c)) or column-to-façade connections (Figure 1 (b))) are realized by laminated connections. Many more structural applications of laminated connections can be found in following Apple Retail stores projects realized worldwide [14]-[16]. Projects with laminated connections have shown over the years increasing complexity in size and geometry, which made them a main reference of glass engineering and architectural detailing. Other applications of laminated connections can be found in the structural façade of the ING Direct building in Barcelona (Spain), in the façade of the IKEA store in the Valladolid (Spain), in the full-glass façade in Switzerland [17], in the Dow Corning European Distribution Center in Feluy (Belgium) [18] and in the upgrade project of the Tottenham Court Road Station, in London (U.K.) [19]. An overview of the research literature on laminated connection is given in the following section TSSA and SG materials 
(a)

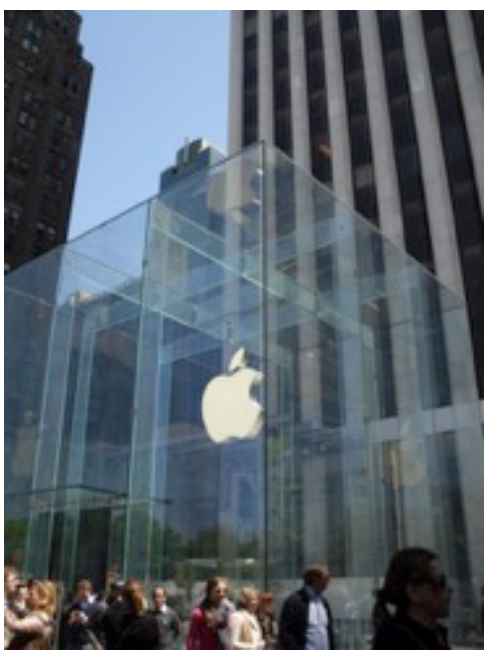

(b)

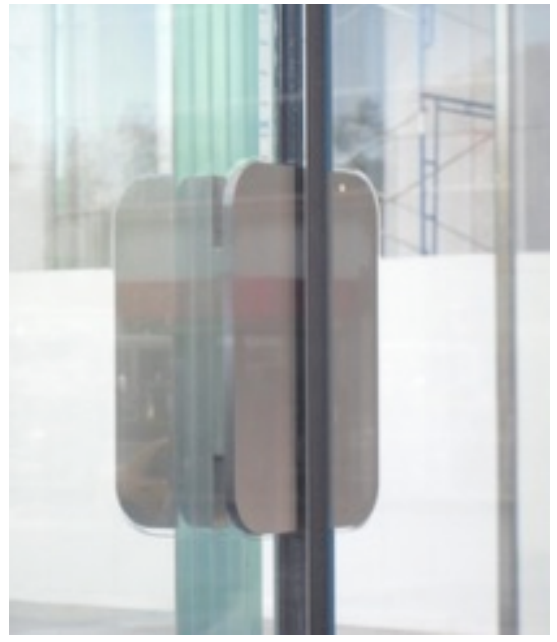

(c)

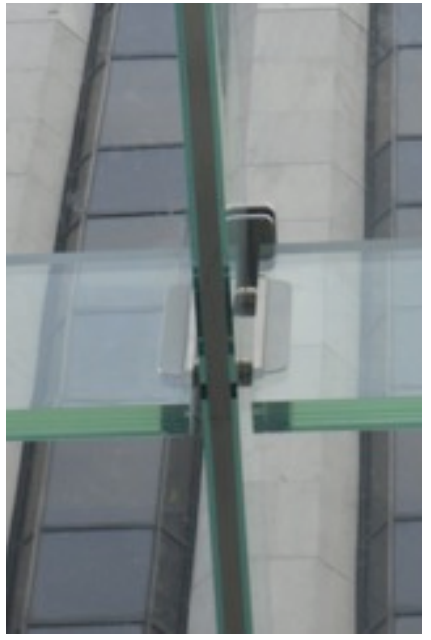

Figure 1: Apple Retail Store at the $5^{\text {th }}$ avenue of New York (U.S.) (a) Global view of the project (b) Close view if the SG laminated connections between column and façade panels (c) Close view of the SG laminated connections between roof beams.

\subsection{Literature on transparent Structural Silicon Adhesive (TSSA) and TSSA connections}

The TSSA material is a Transparent Structural Silicon Adhesive elastomer, produced by Dow Corning, that has been recently commercialized for laminated connections in structural glass applications. Compared to standard silicone adhesives used in glass applications, TSSA exhibits higher stiffness and strength, which makes it suitable for structural applications. It should be noticed that, conversely to SG, TSSA is exclusively intended to realize metal-to-glass adhesive laminated connections bonded to the glass surface. TSSA is, at the time of writing, not intended for laminated glass components in general but rather specifically for laminated connections. A peculiar characteristic of this material is the so-called whitening effect. The TSSA goes indeed from fully transparent to white colour when the stress in the material overcome a certain value and goes back to transparent when stress is removed ${ }^{1}$.

Exploratory experimental investigation on TSSA laminated connections at room temperatures can be found in literature. In the work of Watson \& Overend [20] experimental investigations on TSSA are performed. More specifically, TSSA single lap joints and T-peel specimens are tested at room temperature. The experimental results indicate large potentiality of the TSSA connections because its mechanical response is characterised by an efficient compromise between flexibility and load carrying capacity. In the work of Sitte et al. [21], [22] circular TSSA connections are tested under tensile and shear load. Tests are mainly performed at room temperature and constant displacement rate. Some exploratory investigations are also performed at different temperature. The preliminary results indicate that temperature has significant effects on the resistance of TSSA connections. TSSA bulk material is also investigated at room temperature and constant displacement rate. In the work of Wolf et al. [23] the aging of TSSA connections is extensively investigated. Connections are exposed to both outdoor exposition and accelerated aging protocols. Accelerated aging protocols involved U.V. radiation, water immersion and high temperature cycling. The results show that the TSSA preforms well against aging exposition, as expected for silicon material because of its high energy SI-O bonds [24]. In some cases, it is even observed that aging exposition induces enhancement of the mechanical resistance of TSSA connections. This could indicate that either some further curing is occurring over time or that the aging effect is quantitatively more limited than the statistical dispersion of the results. In the work

\footnotetext{
${ }^{1}$ This effect is dependent on the configuration of the three-dimensional stress tensor [70]
} 
of Hagl et al. [25], [26] the mechanical behaviour of circular TSSA connections are experimentally investigated with particular focus on the whitening phenomenon. Tests are performed at room temperature and constant displacement rate. Aging and cycling tests are also performed. The results show that the whitening phenomenon is occurring at consistent value of load. Furthermore, the experimental observations also show that the location of the whitening depends on the diameter and adhesive thickness. More analytical studies and accurate numerical investigation appear necessary to provide a mechanical interpretation to this effect.

\subsection{Literature on ionomer SentryGlas ${ }^{\circledR}(\mathrm{SG})$ and $\mathrm{SG}$ connections}

Several authors have investigated the mechanical response of SG-laminated components [27]-[36] and the SG-bulk material [37]-[44]. Conversely, studies on the resistance of SG laminated connections are rather limited. Exploratory tests on SG laminated connections bonded to the glass surfaces are performed by Peters in [45]. In [45], a rectangular metal connector is bonded to the surface of a laminated glass panel. Tests are then performed clamping the glass panel and applying tensile force to the metal connection. Tests are performed at room temperature. In the work performed by Belis et al. [46]-[48] a broad screening of adhesive connections is performed via a large experimental campaign to select promising adhesives for glass applications. Tests are performed on aluminium-glass single lap joints at reference condition and after exposition to artificial aging protocols (4 and 12 weeks exposition to $90 \%$ R.H and $50^{\circ} \mathrm{C}$ ). Tests were performed at room temperature. Based on the experimental observation, SG connections have been indicated, among others, as a promising candidate for adhesive connections. In the work of Watson and Overend [20], an extensive work is performed testing single lap connections with different adhesive and interlayers, among which SG. The results showed that SG connections exhibit one of the largest load carrying capacity, often limited by glass failure. Limited results are available in literature at different temperatures [16], [44], [49][52]. Preliminary results show that temperature has a dominant effect on the mechanical response of the connection, with a severe reduction of the maximum load-carrying capacity at high temperature. Results also showed that, at room temperature, the maximum capacity of the connection can, depending on the connection geometry, be limited by the plastification of the metal part or by glass breakage.

\subsection{Objectives}

The preliminary investigations available in literature indicated that indicates that SG and TSSA shows a complex behaviour dependent on strain rate and temperature. However, despite their use in several projects, limited information is available on their mechanical resistance and on the effects of strain rate and temperature variation. The aims of this work are therefore (i) to increase the understanding of the mechanical behaviour and strength of this connection typology under tensile loading and (ii) to quantify the effects and strain rate and temperature on the mechanical strength of the connections. This is done by means of an experimental, analytical and numerical study on laminated connections made of circular metal connectors bonded to rectangular glass plates by means of either TSSA or SG adhesive foils.

Firstly, in sections from 2 to 4, TSSA and SG laminated connections are investigated through an extensive experimental campaign. The specimens are loaded in tensile loading up to failure at different temperatures and displacement rates.

Secondly, in section 5, analytical studies are performed to provide a mechanical interpretation to the different phenomena observed during the experimental campaign. Particular attention is given to the effect of the confinement state on the adhesive stress state of the adhesive.

Thirdly, in section 6, three-dimensional numerical analyses of laminated connections are performed by means of finite element method. This is done to quantitatively study the non-linear field distribution in 
the connection, focusing on the stress peaks occurring in the adhesive and on the triaxiality of the stress state.

Finally, in section 7, failure prediction models are proposed for the tensile resistance of TSSA and SG laminated connections. The models provide the connection resistance with analytical expression function of the applied strain rate and temperature. 


\section{Materials and methods}

\subsection{Materials}

Table 1 collects a summary of the basic properties provided by standards and material producers for the materials used in this study, which are SG, TSSA, glass and stainless steel. Further material properties used in this work are taken for the experimental investigation performed in [44].

Table 1: Materials properties provided by standards and material producers.

\begin{tabular}{lllllll}
\hline Property & Density & $a_{T}$ & $E$ & $v$ & $s_{\max }$ & $e_{\max }$ \\
\hline Unit & $\mathrm{g} / \mathrm{cm}^{3}$ & $10^{-5} /{ }^{\circ} \mathrm{C}$ & $M P a$ & - & $M P a$ & $\%$ \\
$\mathrm{SG}^{a}$ & 0.95 & $15-10$ & $692-0.5$ & $0.5-0.4$ & 34.5 & 400 \\
$\mathrm{TSSA}^{b}$ & $\mathrm{n} / \mathrm{a}$ & $\mathrm{n} / \mathrm{a}$ & $9.0-4.5$ & $\mathrm{n} / \mathrm{a}$ & 8.5 & 250 \\
$\mathrm{Glass}^{c}$ & 2.50 & 9 & 70000 & 0.23 & $45^{e}$ & 0.06 \\
Stainless steel 316L 1.4404 $^{d}$ & 7.85 & 16 & 200000 & 0.3 & $530^{f}$ & 40 \\
\hline
\end{tabular}

a) [53][29] b) [54] c) Soda lime silicate float glass [55]-[57] d) [58], [59] e) characteristic equi-biaxial bending stress at $2 \mathrm{MPa} / \mathrm{s} \mathrm{f}$ ) ultimate stress

\subsubsection{Transparent Structural Silicon Adhesive (TSSA)}

TSSA is a one-component addition-cured silicon with no by-products, characterized by nano-silica and cross-linked polymers. The curing chemical reaction occurs between Si-H containing polymers and Si-Vinyl containing polymer in the presence of platinum with addition cure hydrosilylation ( $\mathrm{Si}-\mathrm{H}$ $+\mathrm{Si}-\mathrm{Vinyl}=\mathrm{Si}-\mathrm{CH} 2-\mathrm{CH} 2-\mathrm{Si})^{3}$. TSSA is characterized ${ }^{4}$ by an amorphous structure since crystallization starts below $-55^{\circ} \mathrm{C}$. The glass transition temperature of the polymers is around $-120^{\circ} \mathrm{C}^{5}$. The stiffness of TSSA is therefore rather stable against temperature variation. Given its aforementioned characteristics, it follows that the use of TSSA adhesive in laminated glass application is quite convenient. Indeed, the autoclave pressure ensures good contact between the adhesive and the adherend surfaces and the heat provided in the lamination cycle activates the addition-cured curing.

TSSA is produced in foils of $1 \mathrm{~mm}$ thickness that are usually approx. $250 \mathrm{~mm}$ wide. Foils are delivered with two protective films on both sides to be removed before application. TSSA should be stored at low temperature to prevent premature curing. TSSA is relatively soft and easy to cut before lamination. The specimens preparation is performed by (i) removal of one of the films and application of the TSSA to the metal connector (ii) cut of the excessive material out of the metal connector (iii) removal of the second film and application to the glass surface and (iv) application of pre-pressure. The producer suggest to apply a pre-pressure in the range of $0.15-1.3 \mathrm{MPa}$ [18]. This helps to prevent air inclusions and to ensure good contact between the TSSA and the adherend surfaces. Both glass and metal connector must be cleaned by means of cleaning agent (e.g. isopropyl alcohol) and silane primer.

\subsubsection{Ionomer Sentryglas ${ }^{\circledR}(\mathrm{SG})$}

SentryGlas ${ }^{\circledR}(\mathrm{SG})$ is a thermoplastic transparent ionomer polymer used in laminated glass applications as interlayer. The glass transition temperature of SG is reported to be around $50-55^{\circ} \mathrm{C}[60][61]^{6}$.

\footnotetext{
${ }^{2}$ It should be noticed that these values are time and temperature dependent.

${ }^{3}$ P.V. Dow Corning Europe, Personal Communication, July $22^{\text {th }}, 2015$.

${ }^{4} \mathrm{Within}$ the common range of temperatures for structural applications, e.g. $-20^{\circ} \mathrm{C}+80^{\circ} \mathrm{C}$ according to ETAG 002 [62]

${ }^{5}$ P.V. Dow Corning Europe, Personal Communication, July $22^{\text {th }}, 2015$.

${ }^{6}$ This is higher than other common interlayer polymers used in laminated components, such as standard PVB with a typical glass transition temperature around $15-20^{\circ} \mathrm{C}$.
} 
Compared to other interlayers such as PVB and EVA, SG is characterized by higher stiffness, enhanced durability and higher mechanical resistance. In the production of laminated glass connections, glass, SG foil and metal parts are placed in a vacuum bag and subjected to an autoclave process $^{7}$. The lamination process consists in a single cycle of simultaneous application of heat and pressure. Typically, a temperature of $135^{\circ} \mathrm{C}$ and a pressure of 12 bar are applied for a minimum plateau time of 60 minutes $^{8}$. Subsequently, to achieve good lamination quality, the cooling phase should be performed with a minimum rate of $2-3^{\circ} \mathrm{C} / \mathrm{min}$. At the end of the autoclave process, the SG material is fully transparent $t^{9}$.

\subsection{Specimens and setup}

The laminated connections tested in this work are obtained by bonding metal connectors to glass plates via TSSA and SG laminated transparent adhesive polymers. The TSSA nominal thickness is $1 \mathrm{~mm}$. The SG nominal thickness is $1.52 \mathrm{~mm}$.

Solid metal connectors are machined out of a solid circular metal bar of $50 \mathrm{~mm}$ diameter, with a height of 20mm and tolerance h9 [ISO 286] (see Figure 2 and Figure 3). The bonded surface is machined to a roughness of 8 micron. A $10 \mathrm{~mm}$ blind threaded hole is machined along the connector axis with a depth of $15 \mathrm{~mm}$. Two main reasons motivated the choice of circular shape for the metal connector. Firstly, a circular shaped connector avoids stress intensification at the corners that occurs, for instance, in rectangular connectors. Secondly, with circular connectors, the orientation and alignment with the glass edge is not critical. This facilitates the production process and the testing of laminated connections. Metal connectors are made of stainless steel $316 \mathrm{~L}^{10}$, a commonly used material for connections in facades and structural glass applications.
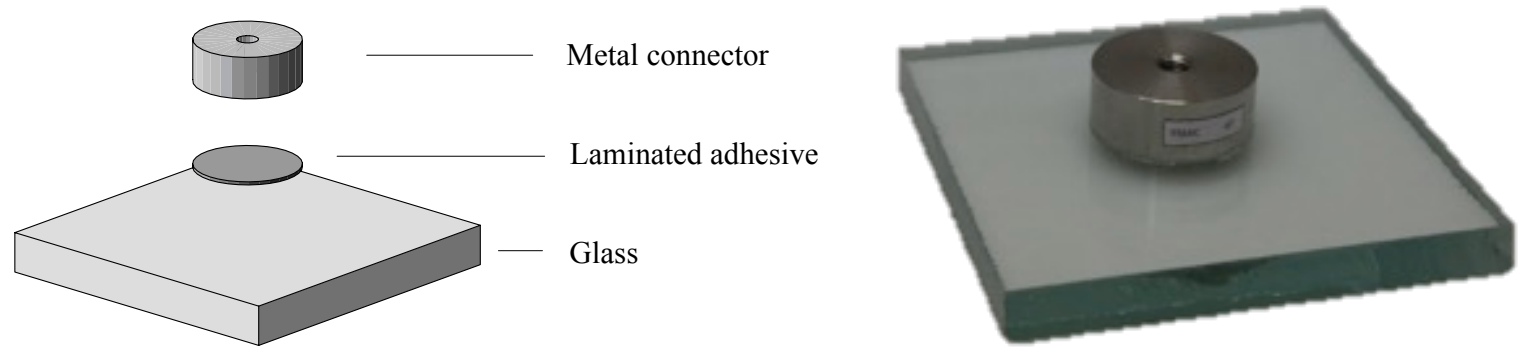

Figure 2: Scheme and photo of the specimen used in the tensile tests

Annealed glass plates of $150 \mathrm{~mm} \times 150 \mathrm{~mm}$ and thickness of $15 \mathrm{~mm}$ are used for TSSA connections. Tempered glass plates of $300 \mathrm{~mm} \times 150 \mathrm{~mm}$ and thickness of $19 \mathrm{~mm}$ are used for SG connections. Analytical calculation and exploratory investigations showed that the use of annealed glass for SG connections would induce glass failure before the failure of the adhesive. This would not allow to obtain information on the adhesive mechanical properties. A $19 \mathrm{~mm}$ tempered glass is therefore chosen to reduce as much as possible the risk of glass failure. The longer dimension of $300 \mathrm{~mm}$ is the minimum length that can typically be tempered in standard glass tempering line due to the distance between transport rollers.

\footnotetext{
${ }^{7}$ As an alternative to the autoclave-vacuum process, silicon bag are also used. In these cases, the components are placed inside a vacuumized silicon bag that is then placed inside a oven.

${ }^{8}$ Material producer suggests that optimum values of temperature and pressure depend on the several factors and vary among different glass manufactures (e.g. autoclave size, panel size, factory, etc...). Therefore, the values mentioned in this manuscript must be considered to be only indicatives. For more details the reader should refer to the material producer or certified glass manufactures.

${ }^{9}$ However, it should be noticed that before lamination the SG foils appear not fully transparent because of the micro-channels intentionally realized on the SG surfaces. These micro-channels reduce the risk of air-bubble inclusion since the air can flow out of the component during the lamination.

${ }^{10}$ The $316 \mathrm{~L}$ alloy is an austenitic stainless steel characterized by a better corrosion resistance than the common 304 . The suffix "L" stands for low carbon (i.e. $<0.03 \%$ ) and indicates better weldability performances. This particular stainless steel has indeed reduced risk of intergranular corrosion related to carbides precipitations at the grains boundaries after welding.
} 
(a)

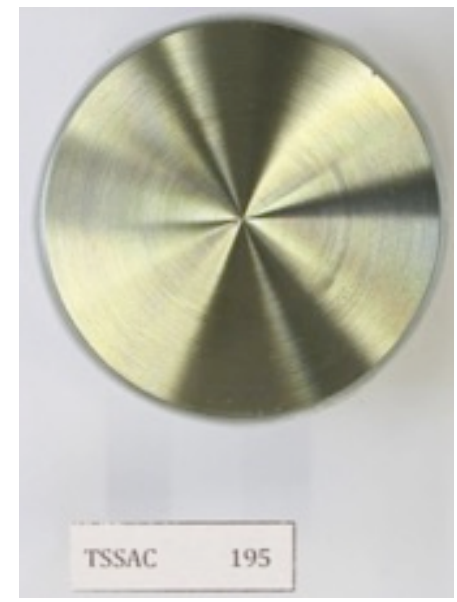

(b)

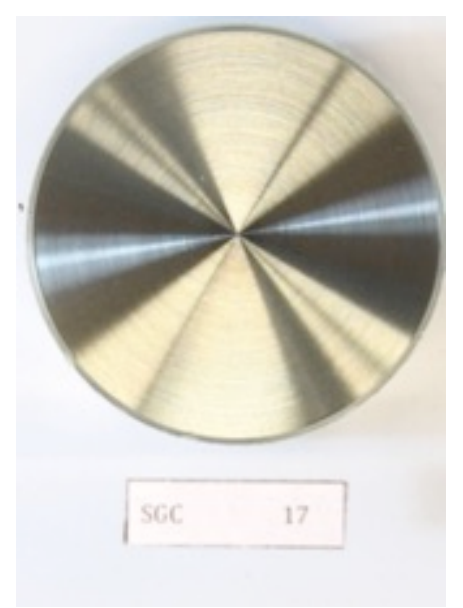

Figure 3: Photo of a (a) TSSA circular laminated connection (b) SG circular laminated connection seen through the glass

Tests are performed with a $50 \mathrm{kN}$ Walter+Bai Universal Testing Machine with a $50 \mathrm{kN}$ load cell. The machine is equipped with a climate chamber of range $-30^{\circ} \mathrm{C}+80^{\circ} \mathrm{C}$ with a resolution of $0.1^{\circ} \mathrm{C}$. A custom made steel setup is fabricated and installed in the machine for the correct introduction of load and to ensure high setup rigidity (see Figure 6 and Figure 7). Tensile load is applied to the specimens by means of a custom made double-hinged metal piece. The two hinges allow rotation about $\mathrm{x}$-axis and y-axis (see Figure 4). The double-hinged metal piece is, at the top, connected to the machine and, at the bottom, attached to the metal connector with a high resistance steel M10 bolt (class 12.9). An aluminium contrast ring is placed between the glass panel and the metal setup. The contrast ring dimensions are as small as possible to minimize glass plate deformation. A minimum distance of $25 \mathrm{~mm}$ between metal connector and contrast ring is required for the instalment of LVDTs. The inner diameter of the contrast ring is thus $100 \mathrm{~mm}$ and the outer is $120 \mathrm{~mm}$. The back plate of the metal setup has a circular hole at the level of the connection to allow visual inspection and video recording of the adhesive through the glass during the test (see Figure 6 and Figure 7 (c)).

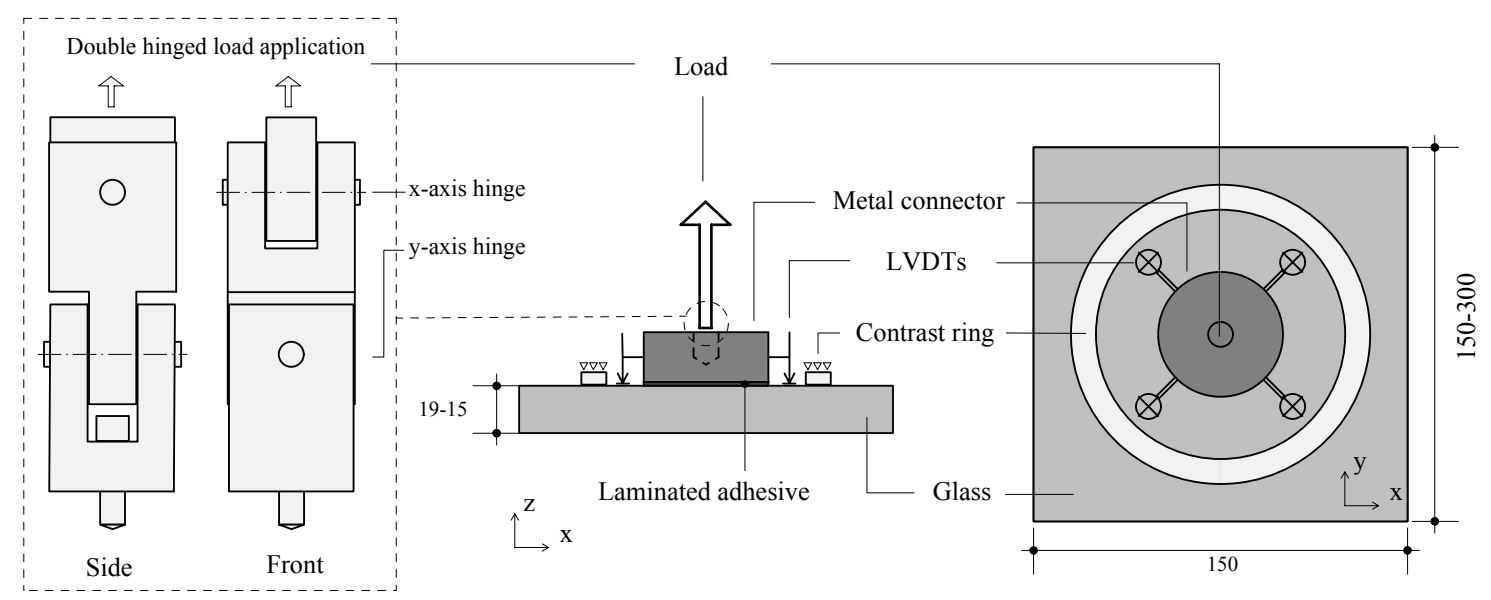

Figure 4: Specimens geometry, load application and boundary condition for tensile tests, dimension in mm.

The load and displacements are measured during the test. Displacements are measured by four inductive LVDTs of $\pm 5 \mathrm{~mm}$, directly attached to the metal connector (Figure 4 and Figure 5). The use of four LVDTs allows to compensate possible rotation due to fabrication tolerances and imperfections. Data are acquired at a frequency of $100 \mathrm{~Hz}$. A video camera is installed inside the climate chamber. The camera is placed on the setup base behind the glass plate (see Figure 6) for video recording of the adhesive during the test. 
(a)

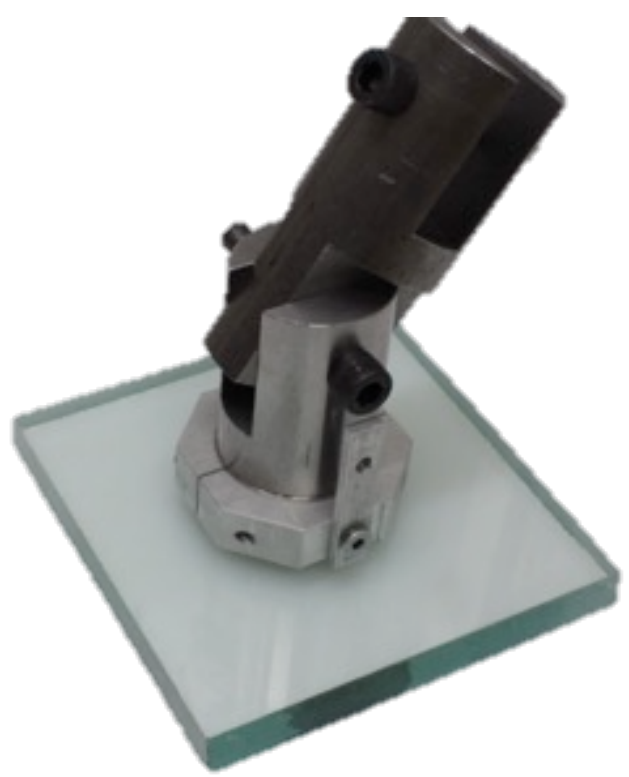

(b)

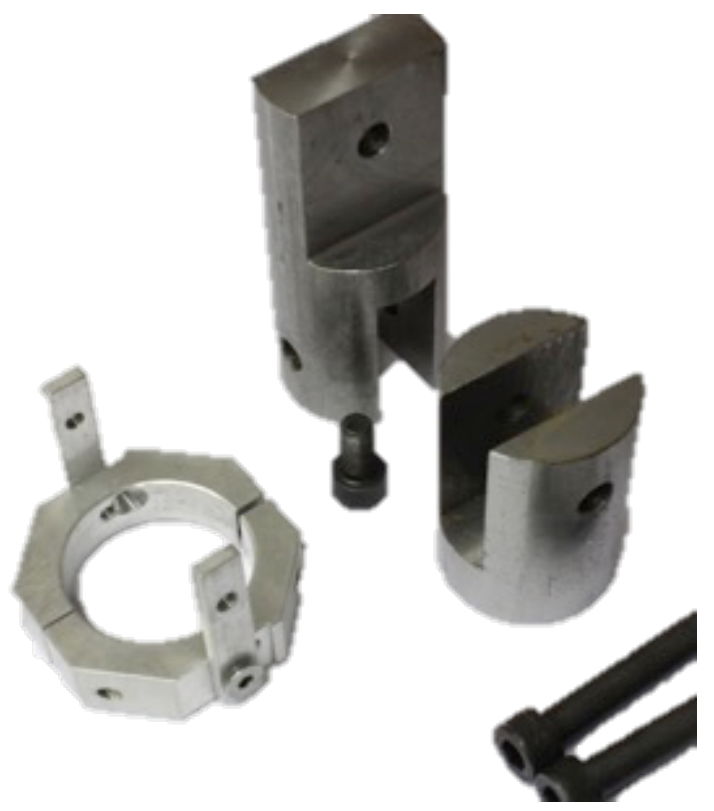

Figure 5: Photos of (a) a laminated connection with the metal fixture for LVDTs (b) exploded view

Exploratory tests have shown non-negligible differences between the cooling-heating rates of the specimen and the climatic chamber. An additional system for additional temperature measurements is therefore required. A total number of five thermocouples were used during each test. The first four thermocouples are used to measure (i) the temperature of the glass close to the adhesive (ii) the temperature of the metal connector close to the adhesive (iii) the temperature of the metal setup and (iv) the temperature of the air in the climatic chamber. This is done of all tested specimens. The fifth thermocouple is laminated within the adhesive in an additional reference specimen. The use of these thermocouples permits to monitor and ensure uniform temperature everywhere during the test. Indeed, each test is started only when each thermocouple has reached the targeted temperature. 


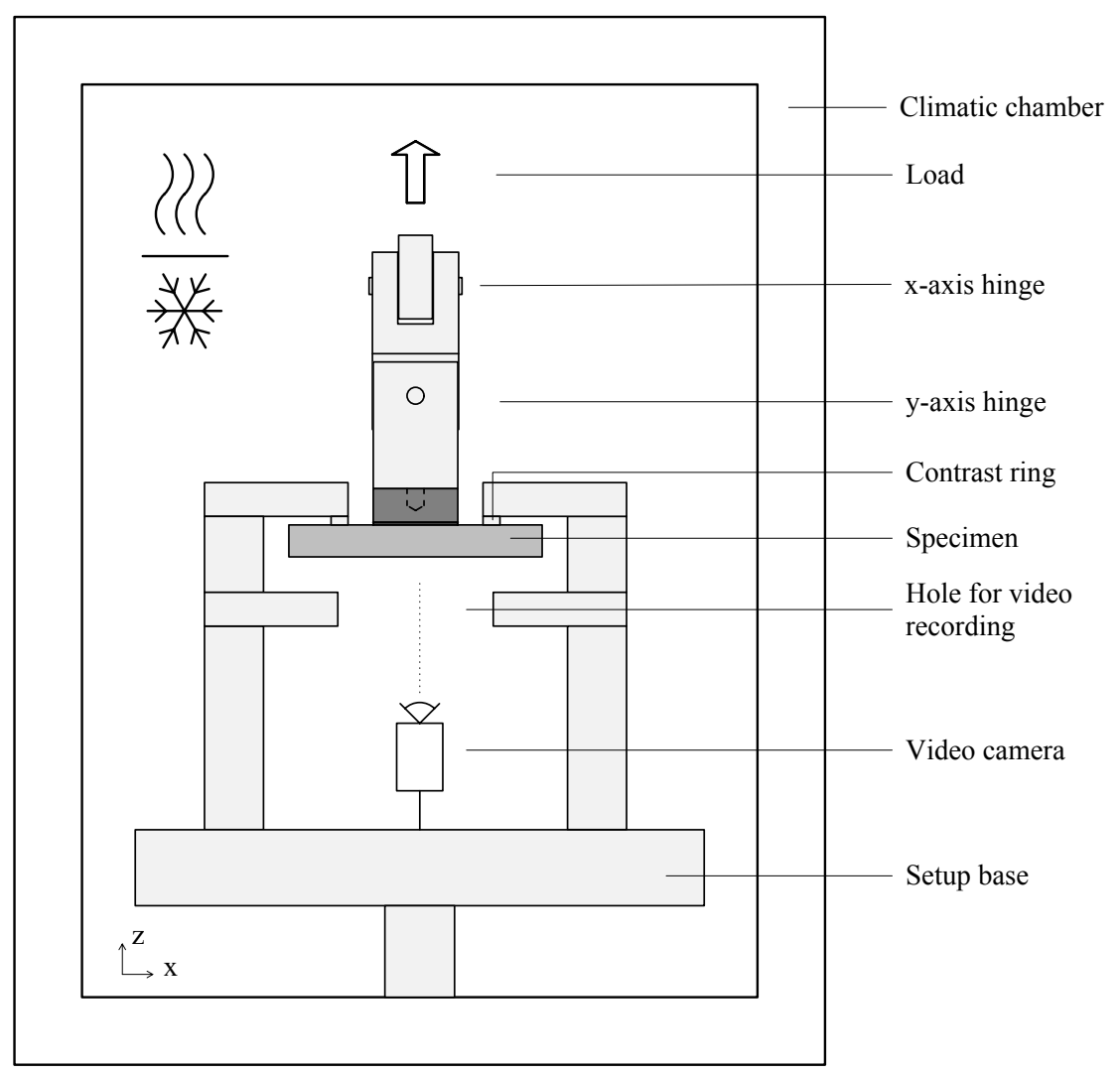

Figure 6: Scheme of tensile test setup

(a)

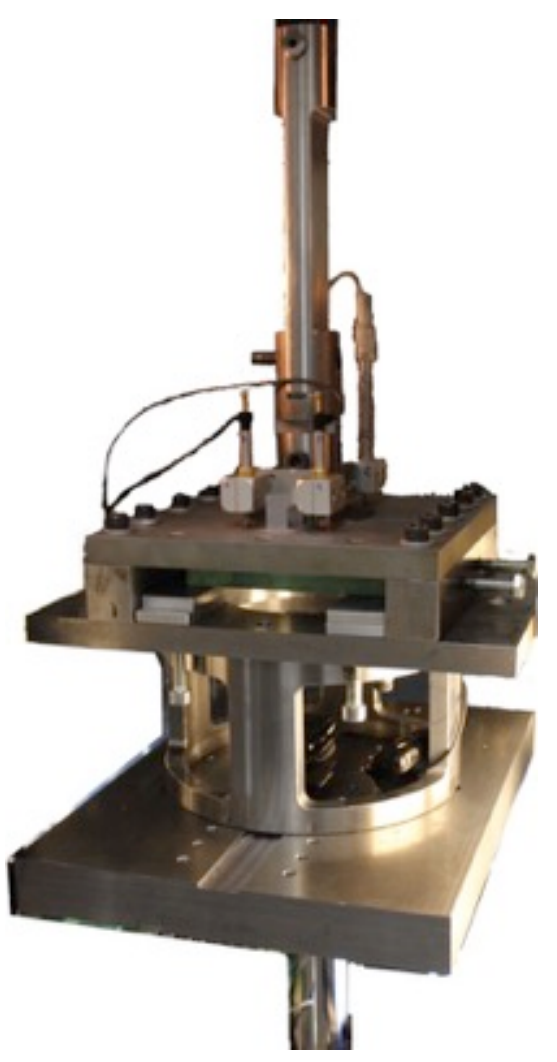

(b)

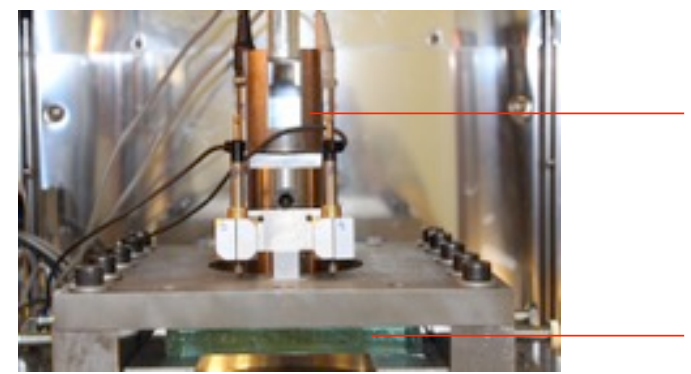

(c)

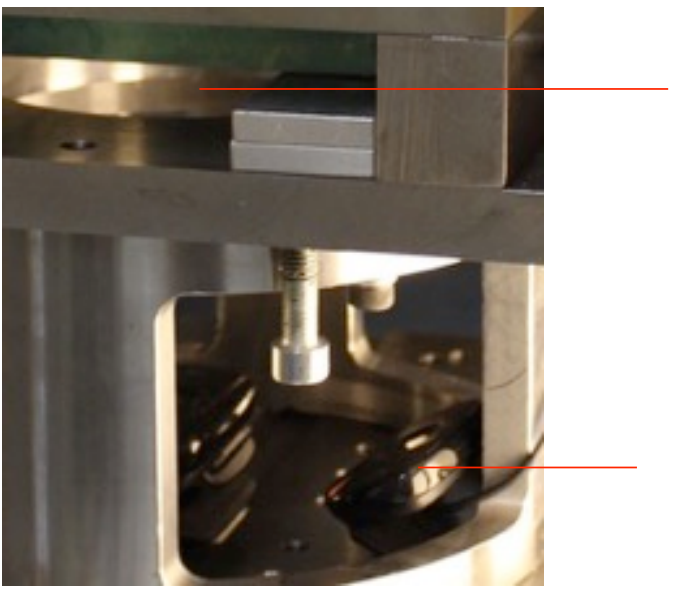

Setup hole for

Video recording

Video camera

Glass panel

Figure 7: (a) Photo of the test setup (b) close frontal view at the connection (c) close view below the glass at the webcam. 


\subsection{Test configurations}

According to guideline ETAG $002[62],-20^{\circ} \mathrm{C}$ and $80^{\circ} \mathrm{C}$ are considered as temperature limits for practical purpose in civil engineering ${ }^{11}$, while $23^{\circ} \mathrm{C}$ is considered as the reference value. According to this indication, TSSA silicon is here tested at $-20^{\circ} \mathrm{C}, 23^{\circ} \mathrm{C}$ and $80^{\circ} \mathrm{C}$. An intermediate temperature of $50^{\circ} \mathrm{C}$ is also tested. SG instead is tested at 7 different temperatures within this range: $-20^{\circ} \mathrm{C}, 0^{\circ} \mathrm{C}$, $23^{\circ} \mathrm{C}, 40^{\circ} \mathrm{C}, 50^{\circ} \mathrm{C}, 60^{\circ} \mathrm{C}$ and $80^{\circ} \mathrm{C}$. SG is tested at more temperatures due to its high temperature sensitivity.

Tests are performed in displacement control. Both materials are tested at three different crosshead machine displacement rates: $0.1 \mathrm{~mm} / \mathrm{min}, 1 \mathrm{~mm} / \mathrm{min}$ and $10 \mathrm{~mm} / \mathrm{min}$. The maximum and minimum displacement rates are defined by practical limitation. Tests at different displacements rates are performed at $23^{\circ} \mathrm{C}, 50^{\circ} \mathrm{C}$ and $80^{\circ} \mathrm{C}$. Tests at $1 \mathrm{~mm} / \mathrm{min}$ are repeated with the same configuration to evaluate statistic dispersion of results (at least five times at $23^{\circ} \mathrm{C}$ and at least three time at the other temperatures). The investigated configurations are summarized by Table 2 . A total number of 53 tests are presented in this work.

Table 2: Test configurations for TSSA laminated connections under tensile load and number of specimens

\begin{tabular}{ccccccccc}
\hline Material & {$[\mathrm{mm} / \mathrm{min}]$} & $-20^{\circ} \mathrm{C}$ & $0{ }^{\circ} \mathrm{C}$ & $23^{\circ} \mathrm{C}$ & $40^{\circ} \mathrm{C}$ & $50^{\circ} \mathrm{C}$ & $60^{\circ} \mathrm{C}$ & $80^{\circ} \mathrm{C}$ \\
\hline \multirow{3}{*}{ TSSA } & 0.1 & - & - & 1 & - & 1 & - & 1 \\
& 1 & 3 & - & 5 & - & 3 & - & 3 \\
& 10 & - & - & 1 & - & 1 & - & 1 \\
\multirow{2}{*}{ SG } & 0.1 & - & - & 3 & - & 1 & - & 1 \\
& 1 & 3 & 3 & 5 & 3 & 3 & 3 & 3 \\
& 10 & - & - & 3 & - & 1 & - & 1 \\
\hline
\end{tabular}

\section{Test results}

\subsection{TSSA under tensile load}

Figure 8 (a) shows the results of TSSA laminated connections under tensile load at different temperatures. The mechanical response of the connection is mainly divided in two branches. Firstly, the connection response is linear and all curves are overlapping, for each investigated temperature. Then, the curve continues with a second phase with an approximately linear behaviour up to failure ${ }^{12}$. Curves are slightly off-set depending on the temperature yet with similar slope. The failure load is observed to be temperature dependent, with larger resistance at low temperature and lower resistance at high temperature. Figure 8 (a) and (b) shows the result of TSSA laminated connection under tensile load at different displacement rates. The general behaviour of the connections remains similar at different displacement rates. However, the displacement rate affects the failure load, with larger resistance at higher displacement rate and lower resistance at lower displacement rate. The actual average strain rate for each displacement rate is computed in the following section.

Figure 9 shows photos of TSSA laminated connections after failure at $-20^{\circ} \mathrm{C}, 23^{\circ} \mathrm{C}$ and $80^{\circ} \mathrm{C}$. All specimens failed cohesively within the adhesive. During each test, a whitening phenomenon is observed. Further analyses of this phenomenon are provided in the following sections. The failure

\footnotetext{
${ }^{11}$ According to ETAG 002 low temperature limit could be extended down to $-40^{\circ} \mathrm{C}$ for European Nordic countries if required [62].

${ }^{12}$ One additional test is performed on a spare sample, unloading the specimens after reaching $90 \%$ of the average failure load. This is done to confirm the hypothesis of hyper-elastic nature of the material as stated in [21]. The result of this test confirm this hypothesis since, once unloaded, the material fully recovered the applied deformations, showing no-plastic residual deformation. However, Mullin's effect's is observed. An additional experimental campaign is suggested to investigate the Mullin's effect at different temperature, displacement rates, number of cycles and load level.
} 
location is at approximately is near the perimeter at $0.8 \mathrm{x}$ the radius measured from the centre of the connection for all investigated temperatures and displacement rates.

(a)

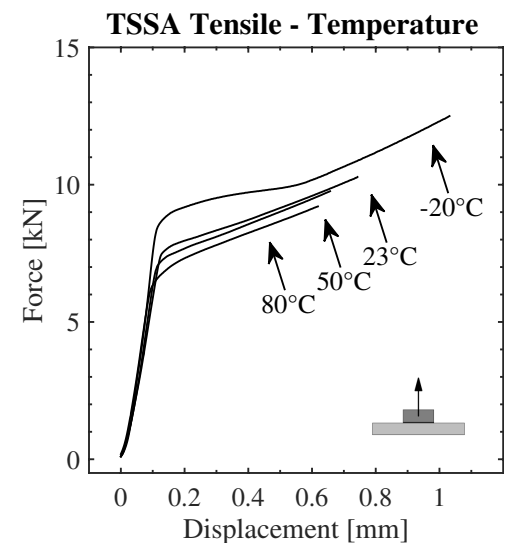

(b)

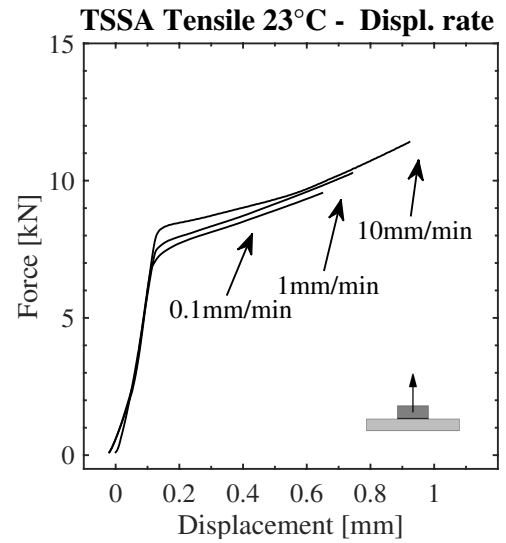

(c)

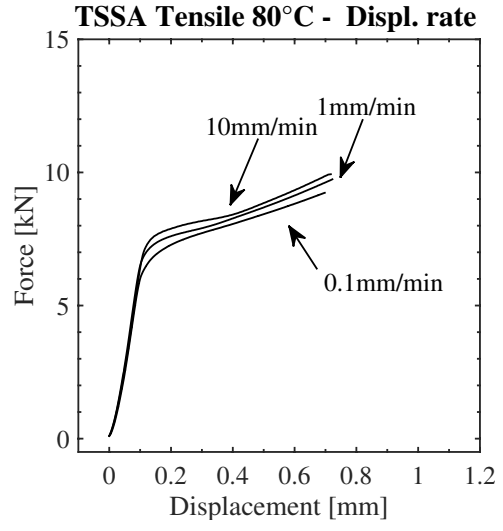

Figure 8: TSSA laminated connections under tensile force (a) Test results at $-20^{\circ} \mathrm{C}, 23^{\circ} \mathrm{C}, 50^{\circ} \mathrm{C}$ and $80^{\circ} \mathrm{C}$ at $1 \mathrm{~mm} / \mathrm{min}$ (b) Test results at different displacement rates at $23^{\circ} \mathrm{C}$ (b) Test results at different displacement rates at $80^{\circ} \mathrm{C}$

(a)

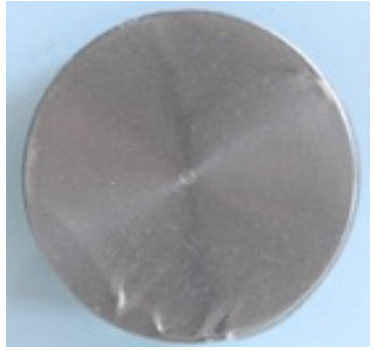

(b)

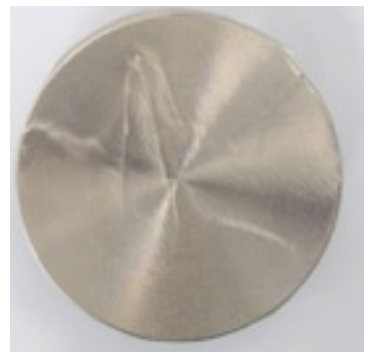

(c)

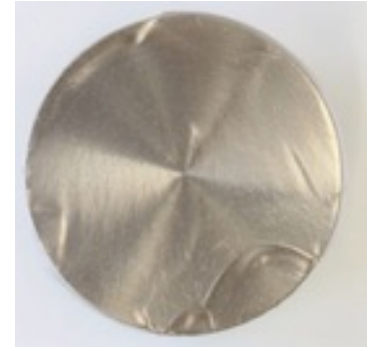

Figure 9: Photo of TSSA tested specimens (a) $-20^{\circ} \mathrm{C}$ (b) $23^{\circ} \mathrm{C}$ (c) $80^{\circ} \mathrm{C}$

\subsection{SG under tensile load}

Figure 10 (a) shows the results of SG laminated connections tested at different temperatures. From $20^{\circ} \mathrm{C}$ to $40^{\circ} \mathrm{C}$ the load displacements curves show a linear response up to brittle failure. At $50^{\circ} \mathrm{C}$, a small deviation from linearity is observed before failure occurs. At $60^{\circ} \mathrm{C}$ and $80^{\circ} \mathrm{C}$, instead, large displacements are measured after the maximum force. The maximum force corresponds to the appearance of bubbles within the material, while the subsequent large displacement is associated to expansion and stretching of the bubbles. Figure 10 (b) and Figure 10 (c) show the effect of displacement rate variation on SG laminated connection. Figure 10 (b) shows that at room temperature the strain rate effect is rather limited. At high temperatures instead (see Figure 10 (c)), the strain rate effect is more pronounced. Namely, larger maximum force is measured at higher displacement rates. 
Figure 11 shows a photograph of an exemplary of SG laminated connection after failure at $-20^{\circ} \mathrm{C}$, $23^{\circ} \mathrm{C}, 60^{\circ} \mathrm{C}$ and $80^{\circ} \mathrm{C}$. The tests results show that the mechanical response and the failure mode of SG laminated connection under tensile load strongly depend on the temperature. For temperature between $-20^{\circ} \mathrm{C}$ and $+40^{\circ} \mathrm{C}$, the material fails cohesively exhibiting a through-thickness fracture plane. The failure is occurring in a localized region close to the perimeter. The through-thickness fracture plane is observed to be inclined with respect to the glass and metal surface. After the adhesive breakage, the fracture propagates adhesively over the connection area either at the glass surface or at the metal surface. Partial glass failure is also observed at room and low temperature. More detailed analyses are provided in the following section. At temperatures above $40^{\circ} \mathrm{C}$, the material fails cohesively in the central part of the connection, in the middle of the adhesive thickness. More specifically, the failure of the adhesive is caused by the formation and expansion of bubbles. This phenomenon is analysed in detail in the following sections.

(a)

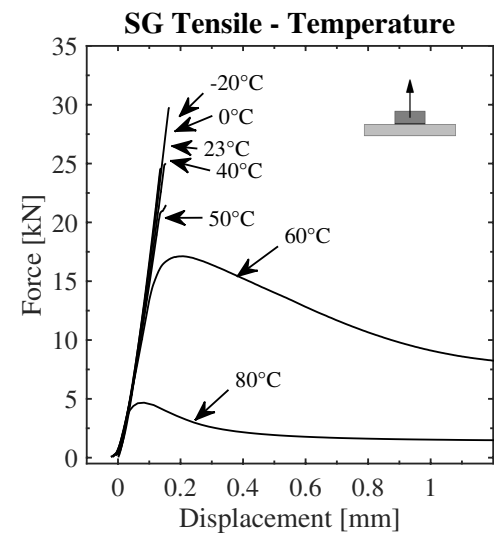

(b)

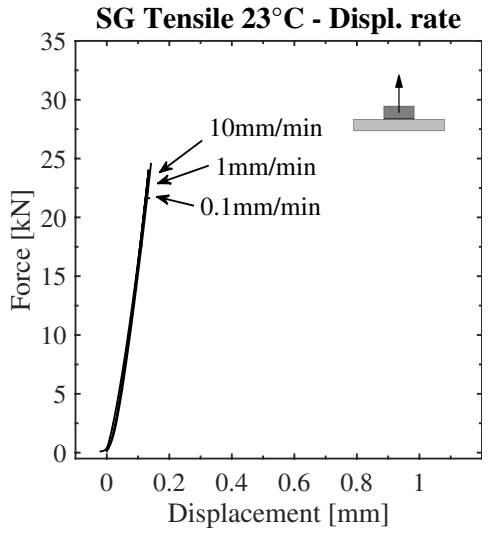

(b)

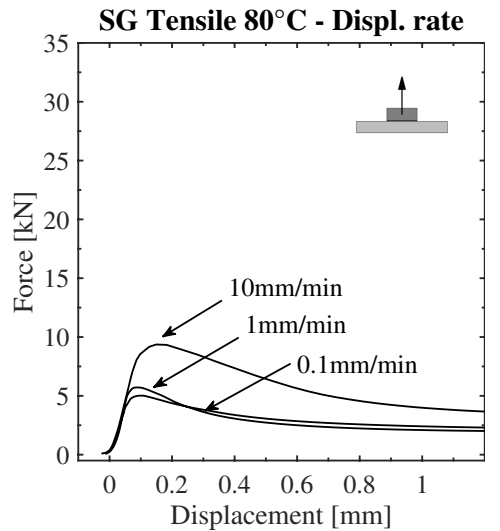

Figure 10: SG laminated connections under tensile force (a) Test results at $-20^{\circ} \mathrm{C}, 0^{\circ} \mathrm{C}, 23^{\circ} \mathrm{C}, 40^{\circ} \mathrm{C}, 50^{\circ} \mathrm{C}, 60^{\circ} \mathrm{C}$ and $80^{\circ} \mathrm{C}$ at $1 \mathrm{~mm} / \mathrm{min}$ (b) Test results at different displacement rates at $23^{\circ} \mathrm{C}$ (c) Test results at different displacement rates at $80^{\circ} \mathrm{C}$

(a)

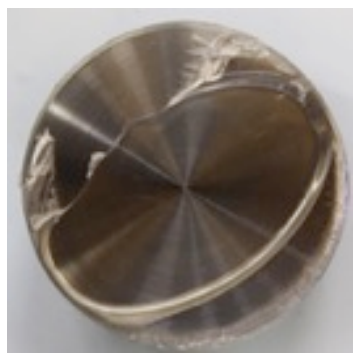

(b)

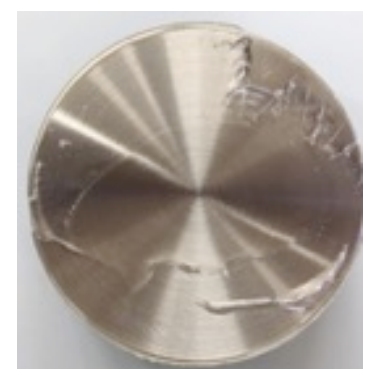

(c)

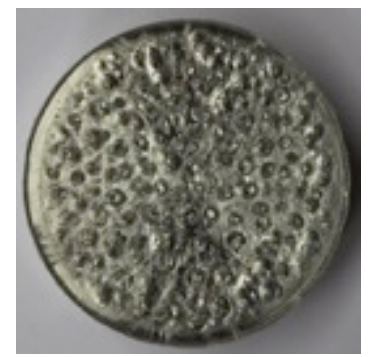

(d)

Figure 11: Photo of SG tested specimens at (a) $-20^{\circ} \mathrm{C}$ (b) $23^{\circ} \mathrm{C}$ (c) $60^{\circ} \mathrm{C}$ (d) $80^{\circ} \mathrm{C}$ 


\section{Tests analysis and discussion}

\subsection{TSSA under tensile load}

Figure 12 (a) shows the results of TSSA laminated connection under tensile load. The resistance of the TSSA is observed to be temperature and strain rate dependent. When compared to the reference condition $\left(23^{\circ} \mathrm{C}\right)$, the resistance increases with $15.4 \%$ at low temperature $\left(-20^{\circ} \mathrm{C}\right)$ and decreases with $12.1 \%$ at high temperature $\left(+80^{\circ} \mathrm{C}\right)$. At different displacement rates, the resistance increases with $10.9 \%$ at $10 \mathrm{~mm} / \mathrm{min}$ and decreases with $13.16 \%$ at $0.1 \mathrm{~mm} / \mathrm{min}$. The resistance appears to follow a linear law with temperature, and a logarithmic law with strain rate variation. However, the strain rate effect is rather limited and in the range of material scatter. Further tests at different displacement rates appear to be necessary to confirm this trend. Table 3 and Table 4 summarise the test results, standard deviations and coefficients of variations. The coefficient of variation of TSSA connection is small (between 0.01 and 0.03 ) indicating small scatter of the mechanical resistance of the connection at any temperature.

(a)

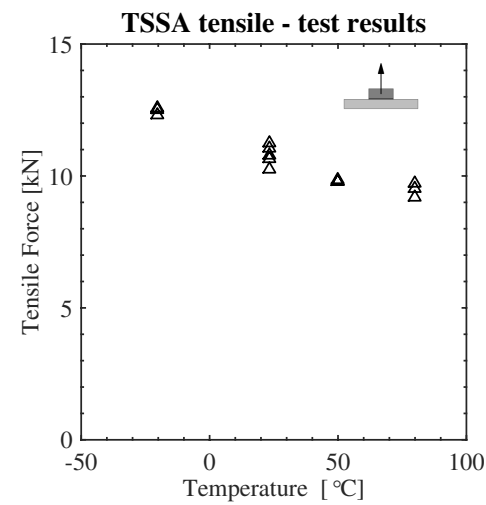

(b)

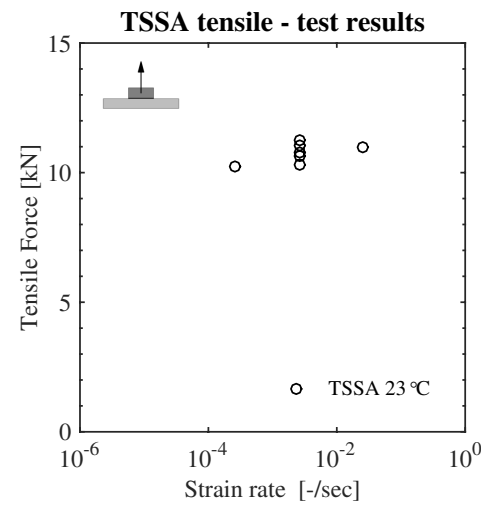

(c)

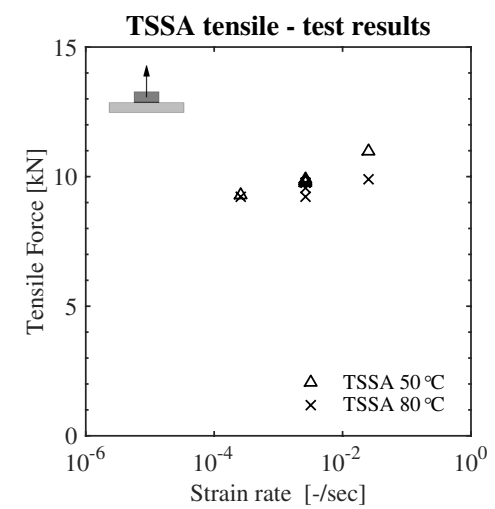

Figure 12: TSSA test results plotted versus (a) temperature (b) strain rate at $23 \mathrm{C} \mathrm{(c)} \mathrm{strain} \mathrm{rate} \mathrm{at} 50$ and $80 \mathrm{C}$

In TSSA connections under tensile force, for each tested configuration, a whitening phenomenon is observed by video camera observation. When the load is applied, the adhesive changes indeed from transparent to white colour. This phenomenon appears to be stress dependent, i.e. occurs at specific load level, $F_{w}$, which depends on the temperature and strain rate. Table 3 and Table 4 collect the whitening load, $F_{w}$, the standard deviations and coefficients of variation for each test configuration. The whitening load is around $45 \%-50 \%$ of the maximum load at failure The initial point of whitening is located approximately at $80 \%$ of the radius (Figure 13 (a)). Then, the whitening rapidly propagates towards the center and slightly towards the perimeter (Figure 13 (b-c)). After this propagation, the whitening phenomenon covers most of the adhesive area (Figure 13 (d)). However, a small ring of adhesive material, i.e. $1-2 \mathrm{~mm}$ close to the perimeter, remains transparent up to failure. After failure, the adhesive goes back to its original transparent state (Figure 13 (e)). The failure location is observed to be at approximately $80 \%$ of the radius for all tested configurations. This indicates that the stress field distribution does not change at different temperatures and strain rates. The comparison of the whitening phenomenon observed in this work under tensile load to the results from uniaxial and shear tests available in literature indicates that the whitening phenomenon is proportional to the hydrostatic component of the stress tensor. Indeed during shear tests [21], [26] the whitening phenomenon is almost not visible and in uniaxial tests [44] it is more visible than in shear tests, yet much less pronounced than in tensile tests. 
(a)

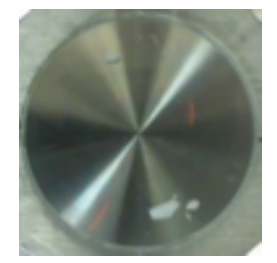

(b)

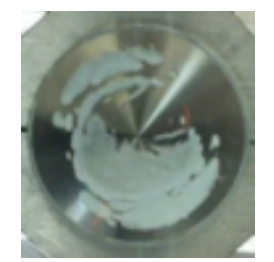

(c)

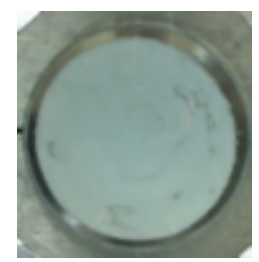

(d)

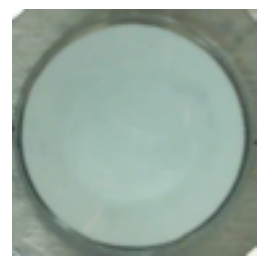

(e)

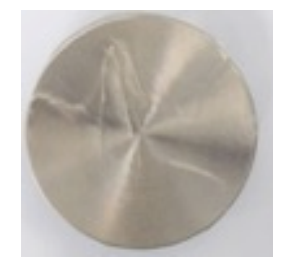

Figure 13: Evolution of whitening in TSSA laminated connection under tensile force (a) beginning (b) quick propagation over most of the adhesive (c) slow propagation close to the perimeter (d) full propagation with a transparent ring close to the perimeter (e) failure

According to these observations, the hydrostatic component ${ }^{13}$ of the stress tensor during the tensile tests is expected to be quite large for most of the adhesive area. Dominant hydrostatic component of the stress tensor could be due to the large diameter-thickness ratio (D/t). Further analytical and numerical analysis are required to confirm this hypothesis. Furthermore, in depth analyses of the stress field distribution are required to provide a mechanical interpretation to the three observed phenomena described in this section: i) the location of the whitening initiation, ii) the presence of a small transparent ring close to the perimeter and iii) the location of final failure.

A possible optical/physical interpretation of the whitening phenomena is the following. Polymeric materials transparent to the visible spectrum of light are usually characterized by an amorphous noncrystallized structure. Amorphous polymer can exhibit, when subjected to tensile loading, the socalled strain-crystallization phenomenon. In strain-crystallization, polymer chains tend to align with each other when stress is applied to the material [63], [64]. Consequently, starting from an amorphous network of polymeric chains, crystals tend to develop (also called lamellae in polymeric material [65][67]). In that state, the light will then scatter at the boundaries of the lamellae crystals. This could explain the whitening phenomenon occurring in the TSSA. This effect, i.e. ordering of amorphous polymer chains network in lamellas, is more favoured by material stretching rather than shearing, which instead induces polymer chain sliding. The whitening is therefore expected to be more pronounced with stress-strain state characterized by a large hydrostatic component of stress tensor, rather than with a dominant deviatoric component. When the applied strain is removed, the polymer chains network tend to partially or fully recover the initial amorphous state, depending on the type of polymer. In hyper-elastic material, such as TSSA, once stresses are removed, the deformations are fully recovered. This could explain the reversibility of the whitening.

Table 3: Test results of TSSA laminated connections

\begin{tabular}{|c|c|c|c|c|}
\hline $\begin{array}{c}\mathrm{T} \\
{\left[{ }^{\circ} \mathrm{C}\right]}\end{array}$ & $\begin{array}{c}\dot{d} \\
{[\mathrm{~mm} / \mathrm{min}]}\end{array}$ & $\begin{array}{c}\dot{\varepsilon} \\
{[-/ \mathrm{sec}]}\end{array}$ & $\begin{array}{c}F_{w} \\
{[\mathrm{kN}]}\end{array}$ & $\begin{array}{c}F_{N} \\
{[\mathrm{kN}]}\end{array}$ \\
\hline-20 & 1 & $2.6 \mathrm{E}-03$ & 6.80 & 12.46 \\
\hline 23 & 0.1 & $2.6 \mathrm{E}-04$ & 4.48 & 10.25 \\
\hline 23 & 1 & $2.6 \mathrm{E}-03$ & 4.63 & 10.80 \\
\hline 23 & 10 & $2.6 \mathrm{E}-02$ & 6.10 & 11.42 \\
\hline 50 & 0.1 & $2.6 \mathrm{E}-04$ & 3.91 & 9.27 \\
\hline 50 & 1 & $2.6 \mathrm{E}-03$ & 4.52 & 9.82 \\
\hline 50 & 10 & $2.6 \mathrm{E}-02$ & 5.30 & 10.97 \\
\hline 80 & 0.1 & $2.6 \mathrm{E}-04$ & 3.80 & 9.24 \\
\hline 80 & 1 & $2.6 \mathrm{E}-03$ & 4.07 & 9.49 \\
\hline 80 & 10 & $2.6 \mathrm{E}-02$ & 4.91 & 9.93 \\
\hline
\end{tabular}

\footnotetext{
${ }^{13}$ A extended study on the stress tensor components and further solid mechanics topics can be found in [70].
} 
Table 4: Test results of TSSA laminated connections - standard deviation and coefficient of variation

\begin{tabular}{|c|c|c|c|c|c|c|}
\hline $\begin{array}{c}\mathrm{T} \\
{\left[{ }^{\circ} \mathrm{C}\right]}\end{array}$ & $\begin{array}{c}\dot{d} \\
{[\mathrm{~mm} / \mathrm{min}]}\end{array}$ & $\begin{array}{c}\dot{\varepsilon} \\
{[-\mathrm{sec}]}\end{array}$ & $\begin{array}{c}F_{w} \\
\text { St.dev }[\mathrm{kN}]\end{array}$ & $\begin{array}{c}F_{w} \\
\operatorname{cov}[-]\end{array}$ & $\begin{array}{c}F_{N} \\
\text { St.dev }[\mathrm{kN}]\end{array}$ & $\begin{array}{c}F_{N} \\
\operatorname{COV}[-]\end{array}$ \\
\hline-20 & 1 & $2.6 \mathrm{E}-03$ & 0.10 & 0.01 & 0.11 & 0.01 \\
\hline 23 & 1 & $2.6 \mathrm{E}-03$ & 0.18 & 0.04 & 0.37 & 0.03 \\
\hline 50 & 1 & $2.6 \mathrm{E}-03$ & 0.36 & 0.08 & 0.06 & 0.01 \\
\hline 80 & 1 & $2.6 \mathrm{E}-03$ & 0.25 & 0.06 & 0.27 & 0.03 \\
\hline
\end{tabular}

\subsection{SG under tensile load}

Figure 14 shows the results of SG laminated connections under tensile load. The resistance of the SG is observed to be temperature and strain rate dependent (see Figure 14 (a) and Figure 14(b-c)). Going from reference temperature $\left(23^{\circ} \mathrm{C}\right)$ to higher temperatures the resistance firstly slightly decreases at $40^{\circ} \mathrm{C}$ and at $50^{\circ} \mathrm{C}$ (with $0.7 \%$ and $13.9 \%$ respectively) and then significantly drops at $60^{\circ} \mathrm{C}$ and at $80^{\circ} \mathrm{C}$ (with $35.5 \%$ and $81.3 \%$ respectively). From reference to low temperature the resistance also decreases with $31.1 \%$ and $14.85 \%$ for $0^{\circ} \mathrm{C}$ and $-20^{\circ} \mathrm{C}$ respectively. At different displacement rates, the resistance increases with $45.14 \%$ at $10 \mathrm{~mm} / \mathrm{min}$ and decreases with $25.3 \%$ at $0.1 \mathrm{~mm} / \mathrm{min}$. The strain rate effect is larger at high temperatures than at room temperature (see Figure 14 (c) versus Figure 14 (b)). One of the reasons contributing to this difference is that at high temperature the cohesive failure is occurring over a large area of adhesive while at room temperature it is concentrated in a local region close to the perimeter (see following Figure 15 versus Figure 18). As for TSSA, the SG strain rate effect also seems to follow a logarithmic law with the strain rate variation. However, this effect appears to be in the range of the results scatter. Further tests at different strain rates appear therefore necessary to confirm this trend. Table 5 and Table 6 summarise the test results, standard deviations and coefficients of variations. Table 5 and Table 6 show that the scatter of the SG results at room and low temperatures is larger than at higher temperatures ${ }^{14}$. In addition, not only the resistance but also the location of failure and the failure mode of SG laminated connections are observed to be temperature dependent.

(a)

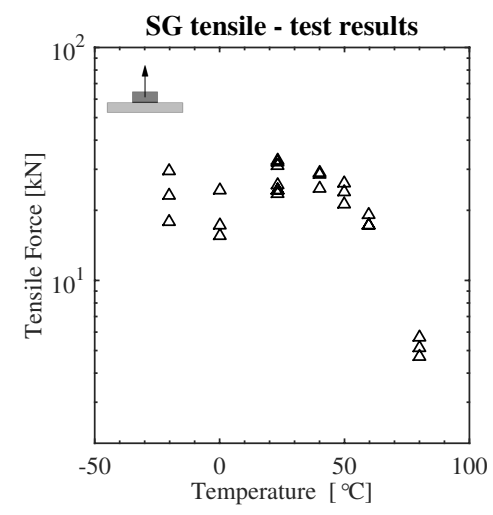

(b)

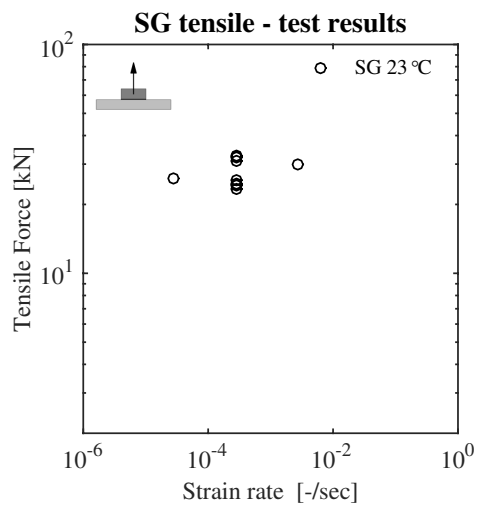

(c)

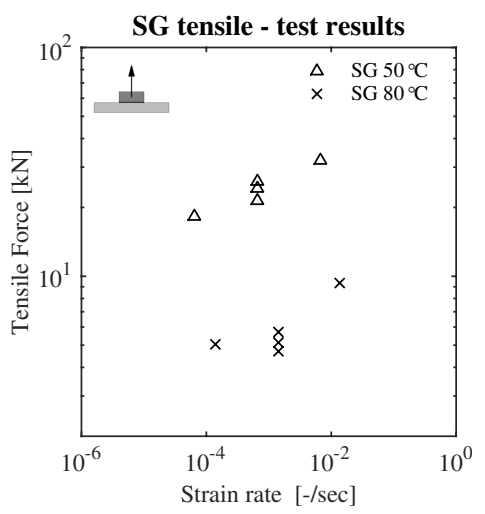

Figure 14: SG test results plotted versus (a) temperature (b) strain rate at $23^{\circ} \mathrm{C}$ (c) strain rate at $50^{\circ} \mathrm{C}$ and $80^{\circ} \mathrm{C}$

At temperatures higher than $40^{\circ} \mathrm{C}$, the failure mode is dominated by the formation of bubbles within the adhesive. Firstly, few bubbles appear in the central part of the adhesive, i.e. far from the perimeter (Figure 15 (a)). Then, the bubbles expand and the number of bubbles increases until most of the

\footnotetext{
${ }^{14}$ One of the causes of this increased scatter could be due to the large sensitivity of rigid adhesives to production tolerances and geometrical imperfection, e.g. inclination of the metal connector and thickness variation. Indeed, at low temperature, the material is rigid and an initial metal inclination, even if small, can induce significant stress peaks at the perimeter. Conversely, at high temperature the material is softer and therefore able to accommodate possible imperfections. Further experimental investigations appear to be necessary to confirm this larger scatter at low temperature.
} 
adhesive area is covered (Figure 15 (c-d)). Here, two main observations are made: (i) the distance between bubbles appear to the rather constant, both along radial and tangential direction, and (ii) a small ring of material close to the perimeter remains bubble-free, i.e. no bubble occurs there. Once the bubbles phenomenon covers most of the adhesive area, the number of bubbles does not increase further. Each bubble expands and then collapses (see Figure 16) due to pressure difference between inside and outside the bubbles. After this phase, the remaining adhesive between collapsed bubbles elongates, resulting in filaments attached to the metal connector and the glass surface (see Figure 17). Finally, when the displacement increases further, the adhesive filaments either progressively break or detach from the adherends $\left(\right.$ at $\left.60^{\circ} \mathrm{C}\right)$ or exhibit extremely large deformation up to machine stroke limit without detachment (at $80^{\circ} \mathrm{C}$, see Figure 15 (e) and Figure 17) (b).

(a)

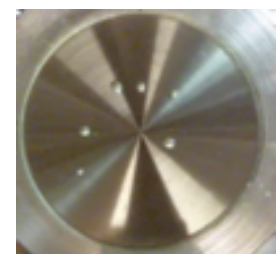

(b)

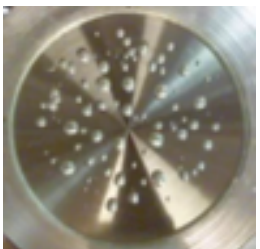

(c)

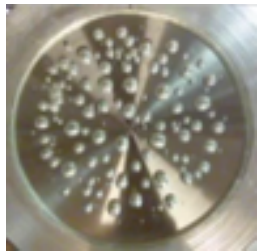

(d)

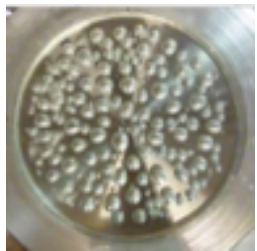

(e)

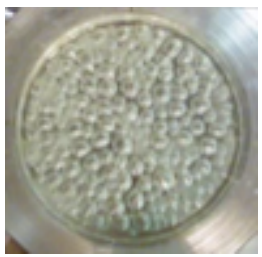

Figure 15: Evolution of cohesive bubble in SG laminated connection under tensile force (a) bubble formation (b-c) bubbles expand and new bubble are formed (d) distance between bubble is constant and number of bubble is stable (e) bubbles collapse and the remaining adhesive material form filaments that elongate

A possible interpretation to the phenomenon of bubble formation is the following. On one hand side, due to the confinement effect induced by the large $\mathrm{D} / \mathrm{t}$ ratio, the stress state of the adhesive is characterized by a large hydrostatic component (see following section for detailed analysis on this topic). This implies that the adhesive material is subjected to a strain tensor that mainly induces volume change. On the other hand side, at high temperature, the SG Poisson's ratio approaches a value of 0.5 [44]. Materials with Poisson's ratio close to 0.5 are also called incompressible material since no-volume change is allowed. This implies that an hydrostatic strain tensor is not admissible. This corresponds to an infinitely rigid mechanical response against pure hydrostatic stress state. According to that, the material must develop bubbles in order to simultaneously full-fill the two conditions, i.e. hydrostatic stress state and almost incompressible behaviour.

(a)

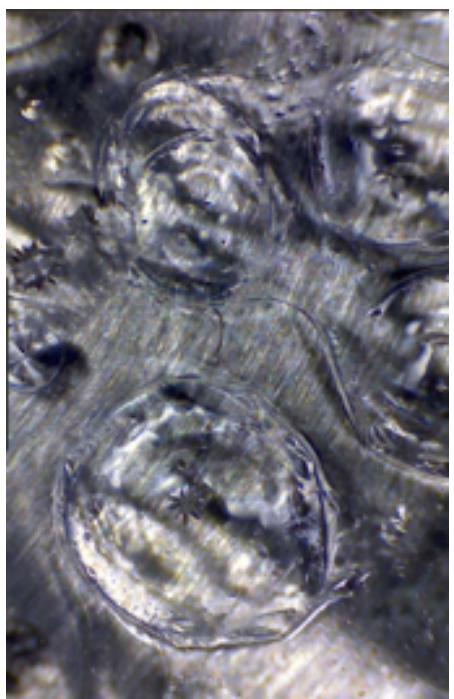

(b)

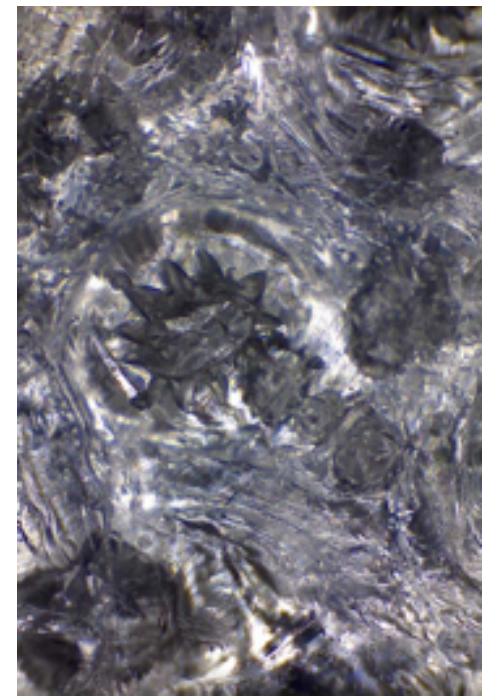

(c)

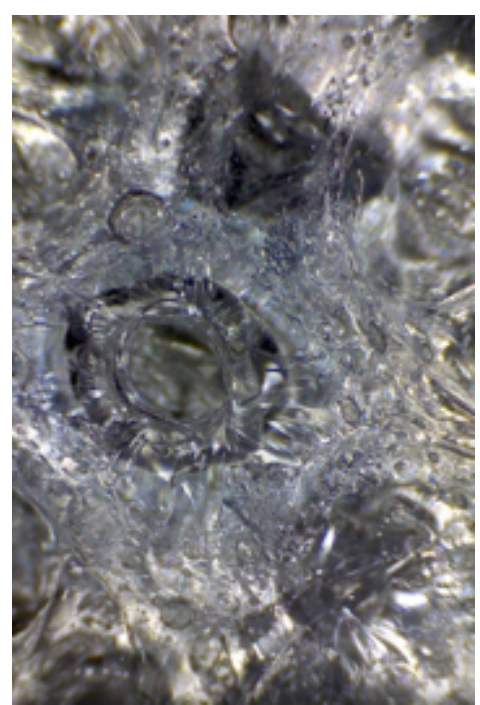

Figure 16: Close view of the bubbles in SG laminated connection under tensile force at $60^{\circ} \mathrm{C}$ 
(a)

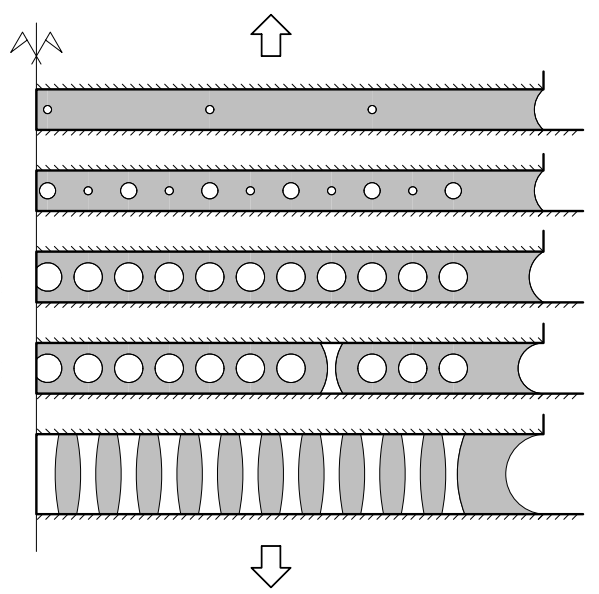

(b)

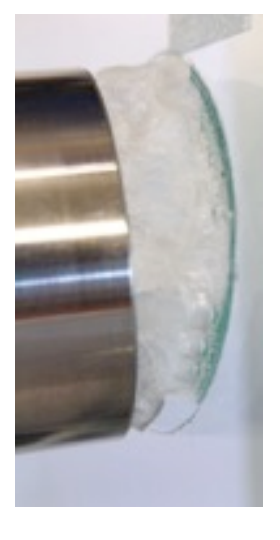

(c)

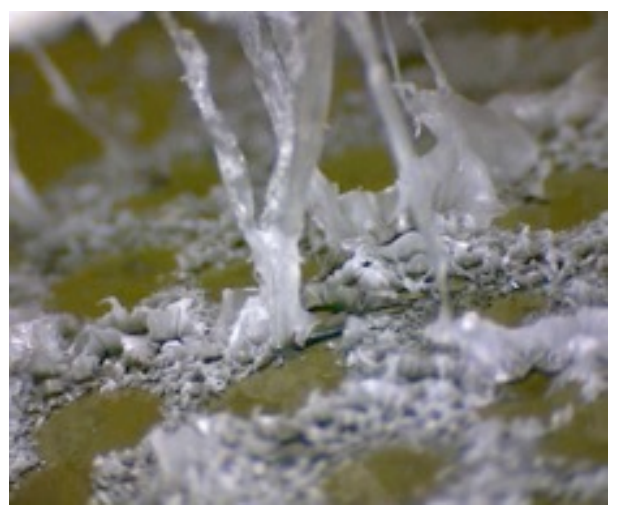

Figure 17: (a) Scheme of bubble phenomenon evolution in SG connection under tensile load at high temperature (b) SG connection after test at $80^{\circ} \mathrm{C}$ (c) close view of SG connection after test at $80^{\circ} \mathrm{C}$

Further detailed analytical and numerical analyses are therefore needed to confirm all the abovementioned considerations. It is indeed necessary to demonstrate that, during the tensile test, the stress state of the adhesive is dominated by a large hydrostatic component. Furthermore, analyses are also needed to provide a mechanical interpretation of the absence of bubble in the small ring of material close to the perimeter.

At room and low temperatures, the adhesive failure is instead located close to the perimeter of the adhesive (see Figure 18). More specifically, failure is initiated cohesively, in a brittle manner at a small localized region close to the perimeter, before progressing adhesively (Fig. 18a). More in detail, an inclined fracture plane through thickness is observed (see scheme of Figure 18 (a)) ${ }^{15}$. The inclined failure plane observed close to the perimeter indicates that at room and low temperature the deviatoric component of the stress tensor is located close to the perimeter and it is larger than at high temperature.

Partial glass failure is also observed at room and low temperature. More specifically, a small 'slice' of glass is plucked off the glass pale. This occurs mainly in the central part of the adhesive (see Figure 18(c)). The glass material pulled out of the panel always remain attached the SG adhesive. The crack so generated in the glass plate does not propagate through the entire thickness and the typical explosive failure of tempered glass is not occurring. The depth of glass chips is in the order of 1-2 $\mathrm{mm}$. From this experimental observation one can consider that the resistance of tempered glass might be not isotropic, i.e. not the same in all three-directions. Consequently, the value of tempered glass resistance provided by standards, typically stated in terms of bending strength, should be used with caution when the principal tensile stress is not laying along the in-plane direction (here indicated as ydirection and $\mathrm{x}$-direction), as it often happens in adhesive connection. This effect could be explained as follows. The tempering residual stresses, which are the cause of higher resistance of tempered glass, are the results of differential cooling times between surface and inner material. During the cooling process, the solidified material at the surface tends to constrain the in-plane contraction of the inner material (along $\mathrm{x}$ and $\mathrm{y}$ directions) causing the typical parabolic profile of compression-tensilecompression residual stresses. However, given the geometry of the problem, the material is free to contract out-of-plane (along z-direction). Therefore, limited residual stresses are built up along zdirection causing the non-isotropy of the residual stresses. The benefit of residual stress might be

\footnotetext{
${ }^{15} \mathrm{At} 23^{\circ} \mathrm{C}$ failure is cohesive, with inclined failure plane, close to the perimeter, At $-20^{\circ} \mathrm{C}$ this failure plane located at the perimeter additionally cohesively propagates over the adhesive forming an ellipse shape
} 
therefore very limited along z-direction. This consideration is of crucial importance with adhesive joints because the vectors of principal tensile stresses are often not oriented along $\mathrm{x}$-axis and $\mathrm{y}$-axis.

As for TSSA and SG at high temperature, further numerical analysis are needed for SG at room and low temperature to confirm that (i) the deviatoric component of the stress tensor is larger than at high temperature and (ii) at room and low temperature that location of maximum stress is close to the perimeter.

(a)

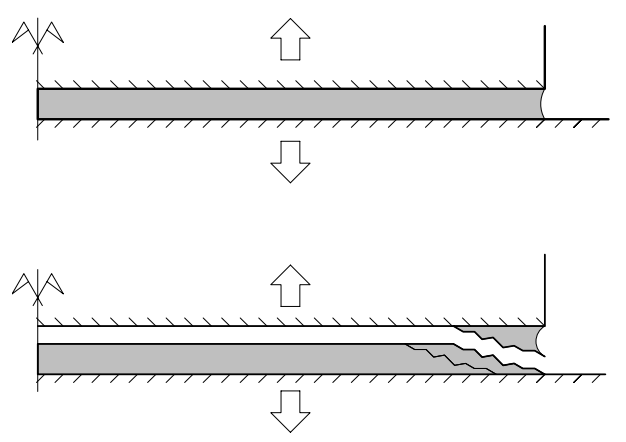

(b)

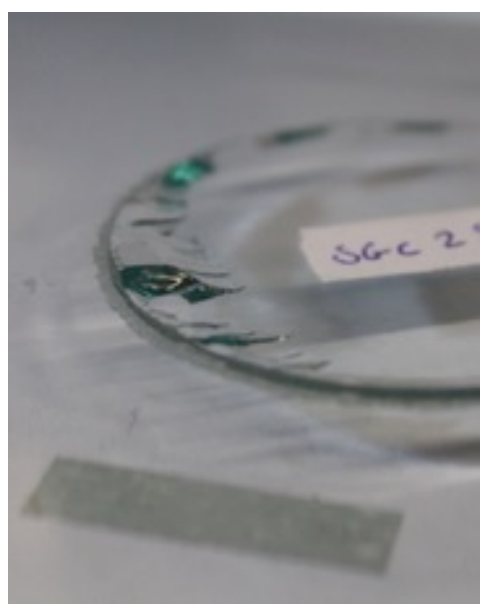

(c)

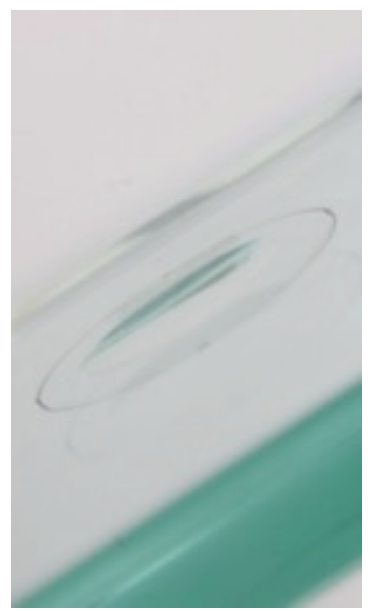

Figure 18: (a) Scheme of SG laminated connection cohesive failure under tensile load at room and low temperatures (b) photo of typical tested SG laminated connection at room and low temperatures (c) glass failure at the bonded surface

Table 5: Test results of SG laminated connections

\begin{tabular}{cccc}
\hline $\mathrm{T}$ & $\dot{d}$ & $\dot{\varepsilon}$ & $F_{N}$ \\
{$\left[{ }^{\circ} \mathrm{C}\right]$} & {$[\mathrm{mm} / \mathrm{min}]$} & {$[-/ \mathrm{sec}]$} & {$[\mathrm{kN}]$} \\
\hline-20 & 1 & $2.2 \mathrm{E}-04$ & 23.60 \\
0 & 1 & $2.5 \mathrm{E}-04$ & 19.09 \\
23 & 0.1 & $2.8 \mathrm{E}-05$ & 26.00 \\
23 & 1 & $2.8 \mathrm{E}-04$ & 27.72 \\
23 & 10 & $2.8 \mathrm{E}-03$ & 29.99 \\
40 & 1 & $5.0 \mathrm{E}-04$ & 27.53 \\
50 & 0.1 & $6.6 \mathrm{E}-05$ & 18.33 \\
50 & 1 & $6.6 \mathrm{E}-04$ & 23.88 \\
50 & 10 & $6.6 \mathrm{E}-03$ & 31.98 \\
60 & 1 & $1.5 \mathrm{E}-03$ & 17.87 \\
80 & 0.1 & $1.4 \mathrm{E}-04$ & 5.04 \\
80 & 1 & $1.4 \mathrm{E}-03$ & 5.17 \\
80 & 10 & $1.4 \mathrm{E}-02$ & 9.42 \\
\hline
\end{tabular}


Table 6: Test results of SG laminated connections - standard deviation and coefficient of variation

\begin{tabular}{ccccc}
\hline $\mathrm{T}$ & $\dot{d}$ & $\dot{\varepsilon}$ & $F_{N}$ & $F_{N}$ \\
{$\left[{ }^{\circ} \mathrm{C}\right]$} & {$[\mathrm{mm} / \mathrm{min}]$} & {$[-\mathrm{sec}]$} & St.dev [kN] & \begin{tabular}{c} 
COV [-] \\
\hline-20
\end{tabular} \\
0 & 1 & $2.2 \mathrm{E}-04$ & 5.91 & 0.25 \\
23 & 1 & $2.5 \mathrm{E}-04$ & 4.73 & 0.25 \\
40 & 1 & $2.8 \mathrm{E}-04$ & 4.14 & 0.15 \\
50 & 1 & $5.0 \mathrm{E}-04$ & 2.24 & 0.08 \\
60 & 1 & $6.6 \mathrm{E}-04$ & 2.39 & 0.10 \\
80 & 1 & $1.5 \mathrm{E}-03$ & 1.15 & 0.06 \\
\hline
\end{tabular}




\section{Analytical study on the stress state and the mechanical response}

The results and analysis of the phenomena observed in the experimental campaign show that the mechanical behaviour of laminated connections under tensile force is rather complex. Further analyses are therefore required to obtain a full understanding of all phenomena occurring during tensile testing.

In this section, the effect of the adhesive confinement on the stress-strain state is firstly analytically analysed. This is done focusing on all three-dimensional components of the stress and strain tensors. Then, the effect of adherend deformability at different temperature is also studied. The aims of these studies are (i) to determine the effect of the confinement state on the adhesive stress tensor, (ii) to evaluate the evolution of the adhesive stress field distribution in the adhesive, (iii) to derive the main parameters involved in the occurring phenomena and (iv) to estimate how they affect the mechanical response of laminated connections.

\subsection{Stress state of the adhesive}

When laminated connections are tested under tensile load, the adhesive material is subjected to a large confined state, since transversal deformations are fully or almost fully constrained. The effects of this confinement on the mechanical response of the adhesive are now studied. In the following paragraph, given the axial symmetry of the problem, stress and deformation are also described using a cylindrical reference system. Therefore, in Figure 19, $\mathrm{x}$-axis is called transversal radial direction and $\mathrm{y}$-axis is also called transversal tangential direction. The longitudinal direction is along z-axis, i.e. parallel to the loading direction.

Due to the axial symmetry, the transversal tangential deformation is fully constrained (i.e. along y-axis in Figure 19). Then, at a certain distance from the perimeter, due to the large diameter-thickness ratio, the transversal radial deformation is also constrained (i.e. along x-axis in Figure 19). In this condition, the application of stress in the longitudinal direction (e.g. along z-axis in Figure 19) induces stresses also in the other two transversal directions and the stress-strain distribution in the adhesive is in general not uniform.

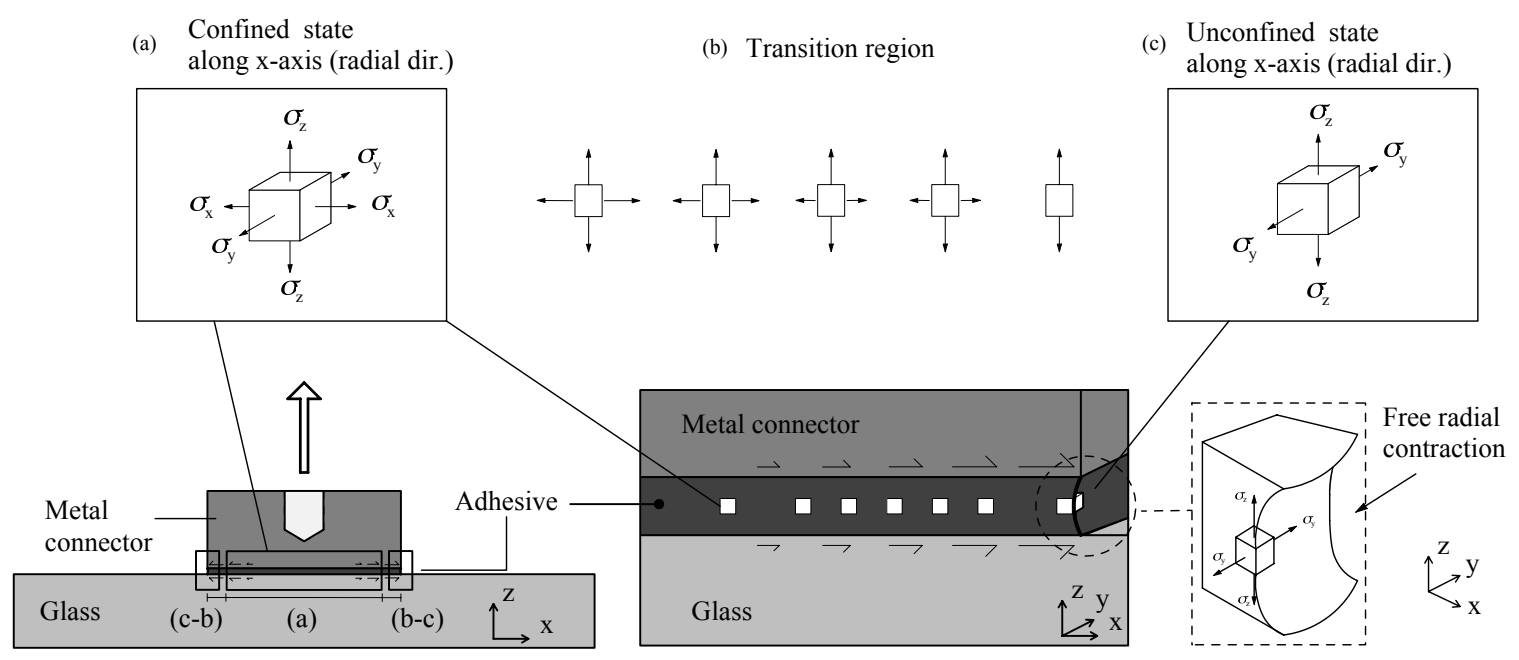

Figure 19: Scheme of the confinement effect and of the three-dimensional stress state of the adhesive

More specifically, three regions can be distinguished (see Figure 19). In region (a), i.e. center part of the connection, the material is fully constrained (both tangentially and radially) and the application of a tensile load to the metal connector induces adhesive stresses in all directions ${ }^{16}$. In region (c), i.e. at the adhesive perimeter, the surface stress-free condition must be satisfied and thus no stresses are

\footnotetext{
${ }^{16}$ It should be noticed that region (a) is expected to cover most of the adhesive material, due to the large D/t ratio. This consideration needs to be validated by non-linear numerical analysis.
} 
developed along radial direction (along x-axis in Figure 19). There, due to the Poisson's effect, the radial transversal deformation is indeed free to occur. Deformations are instead still constrained along tangential direction (y-axis of Figure 19)). Region (b) is a small transition region between (c) and (a). There, moving from (c) to (a), the radial stress increases going from zero to the non-zero stresses in region (a). The transition region is rather limited since the stresses develop with large gradient over the radial direction, due to the large $\mathrm{D} / \mathrm{t}$ ratio. According to these considerations, the mechanical response and the transversal stresses in the adhesive are expected to be function of the applied load and of the Poisson's ratio. The stress state is now analytically analysed in order to validate these considerations. The hypothesis of rigid adherends is here assumed.

Due to the confinement effect, the full three-dimensional stress and strain tensors must be used to analytically evaluate the stress state of the adhesive. Making use of their symmetry, strain and stress tensors can be vectorized and expressed in the Voigt's notation. The relationship between each component of the strain and stress tensors is then expressed by equation (1). The strain tensor is calculated as matrix product of the compliance matrix with the stress tensor. Taking in consideration the proper boundary conditions (equation (2)) in equation (1), a system of two equations is obtained (equation (3)) and equation (4) is then derived. In (3), $E$ is the modulus of elasticity, $v$ is the Poisson's ratio, $\sigma_{z}$ is the applied nominal stress (calculated as the applied force, $F$, divided by the adhesive area, $A), \sigma_{x}$ is the radial stresses and $\sigma_{y}$ is the tangential stress.

$$
\begin{gathered}
{\left[\begin{array}{l}
\varepsilon_{x} \\
\varepsilon_{y} \\
\varepsilon_{z} \\
\gamma_{z y} \\
\gamma_{z x} \\
\gamma_{y x}
\end{array}\right]=\frac{1}{E}\left[\begin{array}{cccccc}
1 & -v & -v & 0 & 0 & 0 \\
-v & 1 & -v & 0 & 0 & 0 \\
-v & -v & 1 & 0 & 0 & 0 \\
0 & 0 & 0 & 2(1+v) & 0 & 0 \\
0 & 0 & 0 & 0 & 2(1+v) & 0 \\
0 & 0 & 0 & 0 & 0 & 2(1+v)
\end{array}\right] \cdot\left[\begin{array}{c}
\sigma_{x} \\
\sigma_{y} \\
\sigma_{z} \\
\tau_{z y} \\
\tau_{z x} \\
\tau_{y x}
\end{array}\right]} \\
\varepsilon_{y}=0 ; \varepsilon_{x}=0 \\
\left\{\begin{array}{l}
\varepsilon_{y}=-v \frac{\sigma_{x}}{E}+\frac{\sigma_{y}}{E}-v \frac{\sigma_{z}}{E}=0 \\
\varepsilon_{x}=+\frac{\sigma_{x}}{E}-v \frac{\sigma_{y}}{E}-v \frac{\sigma_{z}}{E}=0 \\
\left\{\begin{array}{l}
\sigma_{y}-v\left(\sigma_{x}+\sigma_{z}\right)=0 \\
\sigma_{x}-v\left(\sigma_{z}+\sigma_{y}\right)=0
\end{array} \rightarrow \sigma_{y}=\sigma_{x}\right.
\end{array}\right.
\end{gathered}
$$

Then, substituting equation (4) in equation (3), equation (5) and (6) are derived. In equation (6), $E_{c}$ is here called confined stiffness. The latter represents the rigidity of the adhesive in a confined state. Finally, substituting (5) in (3), equation (7) is obtained ${ }^{17}$. Equation (7) describes the magnitude of the confinement stresses occurring in the adhesive when the nominal vertical stress $\sigma_{z}$ is applied.

$$
\begin{gathered}
\varepsilon_{z}=\frac{1}{E}\left(\sigma_{z}-\sigma_{z} \cdot \frac{2 v^{2}}{(1-v)}\right)=\frac{\sigma_{z}}{E}\left(\frac{(1+v)(1-2 v)}{1-v}\right) \\
E_{c}=E \cdot\left(\frac{1-v}{(1+v)(1-2 v)}\right) \\
\sigma_{y}=\sigma_{x}=\sigma_{z} \cdot \frac{v}{(1-v)}
\end{gathered}
$$

From equations (6), (7) and (4) it is stated that due to the confinement effect (i) the adhesive exhibits an increased stiffness, which is in general larger than the modulus of elasticity, (ii) radial and tangential stress are larger than zero and proportional to the applied longitudinal stress and (iii) radial

\footnotetext{
${ }^{17}$ Similarly, the confined stiffness and the tangential stress can be obtained at the perimeter region (c) (not presented here for the sake of brevity). In this case, different boundary conditions must be applied ( $\varepsilon_{y}=0$ and $\left.\sigma_{x}=0\right)$, and the problem solved accordingly.
} 
and tangential stresses have the same magnitude which results in large hydrostatic component of the stress tensor.

The effects of the confinement state are described by analytical expressions function of the Poisson's ratio. This is in agreement with the consideration made at the beginning of this section. In particular, it can be observed that when the Poisson's ratio tends to 0.5 , the confined stiffness tends to infinity and the transversal stresses tend to assume the same value as the applied longitudinal stress. This follows what is expected since Poisson's ratio equal to 0.5 describes an incompressible material.

Going from region (a) to region (c), Equation (6) indicates that the mechanical response of the adhesive material is not uniform over the connection area. Indeed, in the center part of the adhesive, where it is confined, the adhesive exhibits stiffer response than at the perimeter, i.e. the confined stiffness. The confined stiffness is a function of the modulus of elasticity and the Poisson's ratio. It follows that, if a uniform displacement field is applied to the metal connector, the stresses in the adhesive are in general non-uniform, and will tend to redistribute over the adhesive area, with the maximum value in the internal part of the adhesive, i.e. in the stiffer region. A transition region is also expected to occur between the central and the perimeter regions, where shear stresses and the deviatoric component of the stress tensor should be larger than zero. Further numerical analyses of the adhesive stress field distribution are therefore required to confirm these results.

Finally, it is also analytically demonstrated that the application of stresses along longitudinal direction induces large adhesive stresses along the two transversal directions (see equation (7)). The transversal stresses are expressed by an analytical equation function of the Poisson's ratio and the applied longitudinal stress. This demonstrates that the stress tensor in the adhesive is characterized by a large hydrostatic component, and confirms the consideration stated in the previous section. Non-linear numerical analyses are now needed to validate these analytical findings and to quantify the stress peak occurring in the non-linear stress field distribution.

\subsection{Adherends deformability}

In the previous analytic study the hypothesis of rigid adherends is adopted (i.e. metal connector and glass panels are infinitively stiff). This hypothesis can be considered valid only when the stiffness of the adhesive is much smaller than the adherends one. However, especially in case of stiff adhesives, further consideration on the adherends' compliance should be made. The aim of this section is to analytically evaluate the effect of the adherends compliance at different temperatures. The results will indicate whether the adherend deformability must be implemented in non-linear numerical analysis or if the simplifying hypothesis of rigid adherends can be used to reduce the size of the numerical model.

In previous section 2.2 it is mentioned that the support is placed as close as possible to reduce the deformation of the glass plate (small contrast ring internal radius). However, even if limited, a nonzero distance between the support and the metal connector must be left for the instalment of LVDTs. When the tensile force is applied, the glass panel is therefore subjected to bending, resulting in small, yet not zero, vertical displacements. Although very limited, the glass compliance must generally be taken in consideration, especially in case of stiff adhesive. This is because the local bending of the glass might have non-negligible effects on the stress field distribution in the adhesive. A simplified analytical system is now used to qualitatively study this phenomenon.

A laminated connection can be considered as a system of two springs connected in series, $k_{l}$, for the adhesive and $k_{2}$ for the glass panel, (see Figure 20). The metal connector is mainly solicited in tension, not in bending, thus its contribution $k_{3}$ is negligible. The stiffness of the adhesive is estimated by equation (8), where the increase of stiffness due to confinement effect must be taken into consideration (i.e. equation (6)). In (8), $R$ is the radius of the metal connector, $t$ is the adhesive thickness, $E$ is the adhesive modulus of elasticity and $v$ is the adhesive Poisson's ratio. Simplified 
hypothesis of uniform stiffness over the adhesive area is here assumed. The contribution of the glass plate is computed by equation (9), (10) and (11) [68], [69] considering an axial-symmetric plate of radius $R_{s}$, simply supported at the edge (i.e. the contrast ring) and loaded with uniform pressure over a concentrically circle of radius $R$. In (9) $R_{s}$ is the inner radius of the contrast ring, $v_{g}$ is the glass Poisson's ratio, $E_{g}$, is the glass modulus of elasticity, $t_{a}$ is the adhesive thickness and $t_{g}$ is the thickness of the glass plate. The global response is then given by equation (12).
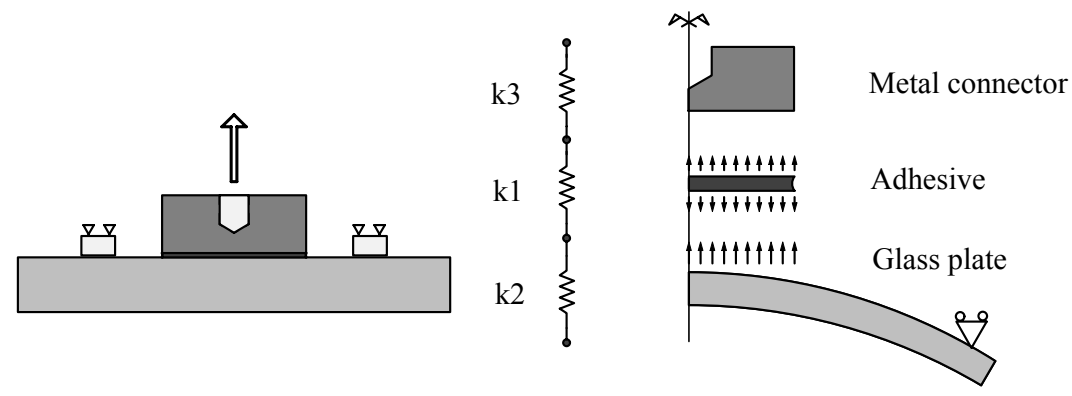

Figure 20: Scheme of equivalent spring model of laminated connection under tensile force, support distance and deformations are amplified for the sake of clarity

$$
\begin{gathered}
k_{1}=\frac{A \cdot E_{c}}{t_{a}}=\frac{E \cdot \pi R^{2}}{t_{a}}\left(\frac{1-v}{(1+v)(1-2 v)}\right) \\
k_{2}=\frac{64 K\left(1+v_{g}\right) \cdot A}{R_{S}^{2} \cdot R^{2} \cdot c} ; k_{3} \gg k_{2}, k_{1} \\
\text { with } K=\frac{E_{g} \cdot t_{g}^{3}}{12\left(1-v_{g}^{2}\right)} ; \beta=\frac{R}{R_{s}} \\
\text { with } c=4(3+v)-(7+3 v) \beta^{2}+4(1+v) \beta^{2} \ln (\beta) \\
k_{\text {tot }}=\left(\frac{1}{k_{1}}+\frac{1}{k_{2}}\right)^{-1}<k_{2}, k_{1}
\end{gathered}
$$

For the sake of example, two extreme cases are now considered using the material properties from [44]: soft adhesive ( $\mathrm{SG}$ at high temperature, i.e. $80^{\circ} \mathrm{C}$ ) and rigid adhesive ( $\mathrm{SG}$ at low temperature, i.e. $20^{\circ} \mathrm{C}$ ). The $k_{2} / k_{1}$ ratio is then calculated for the two cases, respectively by (13) and (14).

$$
\begin{aligned}
& S G \text { at } 80^{\circ} \mathrm{C} \rightarrow \frac{k_{2}}{k_{1}} \cong 6.17 ; \frac{k_{\text {tot }}}{k_{1}}=0.86 \\
& S G \text { at }-20^{\circ} \mathrm{C} \rightarrow \frac{k_{2}}{k_{1}} \cong 0.15 ; \frac{k_{t o t}}{k_{1}}=0.13
\end{aligned}
$$

From equation (13) and (14) it is observed that at high temperature the adhesive deformability is much larger than the glass one. At low temperature, instead, the glass component stiffness is smaller than the adhesive one. This indicates that the glass component deformations in z-direction are in the same order of magnitude or even larger than the adhesive one. In this case, the local deformation of the glass panel modifies the strain distribution in the adhesive, i.e. inducing strain intensification close to the perimeter. Based on these results it is therefore expected that (i) in case of soft adhesive the effect of the glass compliance is limited resulting in a uniform stress distribution field of the adhesive deformation, and (ii) in case of rigid adhesive the glass deformation has a non negligible effect on the adhesive stress distribution since strain intensification is occurring close to the perimeter, and the 
strain distribution is therefore expected to be non-linear. In order to validate the analytical results, comparative numerical analyses are performed in the following section 6.2.2.

From the current analytical studies, it is concluded that the stress distribution in the adhesive is obtained as a sum of two phenomena: a non-uniform confined stiffness over the adhesive area and a strain intensification close to the perimeter. These are respectively due to the confinement state and to the glass deformability. The first phenomenon leads to stress redistribution towards the middle of the connection where the response is stiffer. The second phenomenon leads to stress intensification close to the perimeter. The location of the maximum longitudinal stresses is therefore expected to vary between the centre and the perimeter of the connection, as a result of the combination of the two above-mentioned phenomena.

The results indicate that, because of the complexity of the stress-strain state and its distribution over the adhesive area, detailed non-linear numerical analyses are needed to quantitatively study the stress distribution in the adhesive, the magnitude of the stress peaks and their location in the adhesive. The results of this section recommend that the geometry, boundary conditions and compliance of the adherends must be implemented in the numerical model as close as possible to the reality. 


\section{Numerical analysis and discussion}

In this section the results of the finite element analysis are presented and discussed. The main aims are (i) to investigate the non-linear stress field distribution in the adhesives at different temperatures (ii) to quantify the stress peaks occurring in the adhesive as a function of the temperature and of the material (iii) to validate the analytical considerations made in the previous sections and (iv) to provide a mechanical interpretation of the phenomena observed in the tests, such as TSSA stress whitening, SG bubble formation and changing failure modes. Firstly, the numerical model used for the finite element analysis is described. Secondly, the stress field distributions computed by non-linear analyses are presented and discussed. Thirdly, the results of the numerical study are compared to the experimental observations for validation. Finally, a set of coefficients is defined, called stress factors, which will be used in the last section of this work.

\subsection{Model geometry, boundary conditions and meshing}

A finite element model of the laminated connections is realized with the software ABAQUS. A twodimensional geometry is implemented making use of the axial-symmetry along the central axis (dashdot line in Figure 21). Although the implemented model geometry is two-dimensional, a threedimensional analysis is performed computing the full three-dimensional stress and strain tensors. Figure 21 shows the model geometry and dimensions. The thickness of the adhesive in the model is $1.52 \mathrm{~mm}$ for SG and $1 \mathrm{~mm}$ for TSSA. The glass thickness is $19 \mathrm{~mm}$ for SG and $15 \mathrm{~mm}$ for TSSA. The SG adhesive is modelled with elasto-plastic material and the TSSA adhesive is modelled with hyperelastic material properties. Material inputs are from [44]. Table 7 collects the values of the Poisson's ratio implemented in the model. Quasi-static step-by-step analyses are performed using iterative implicit solver algorithm. Finite deformation theory is used to account for geometrical non-linearity and large deformation.

Table 7: Values of the adhesives Poisson's ratio implemented in the non-linear finite element numerical analyses

\begin{tabular}{ccccccccccc}
\hline Material & \multicolumn{1}{c}{ SG } & \multicolumn{1}{c}{ TSSA } \\
\hline $\mathrm{T}\left[{ }^{\circ} \mathrm{C}\right]$ & 80 & 60 & 50 & 40 & 30 & 23 & 0 & -20 & any \\
$v[-]$ & 0.49 & 0.49 & 0.48 & 0.48 & 0.44 & 0.43 & 0.41 & 0.40 & 0.45 \\
\hline
\end{tabular}

The boundary conditions and load application are implemented as close as possible to the testing setup. The displacements of the aluminium contrast ring along z-axis are constrained. Then, contact ${ }^{18}$ interface interaction is placed between the contrast ring and the glass panel. The tensile load is applied to the metal connector as close as possible to the testing condition, i.e. the load is applied at the perimeter of the drilling. A multiple-points constrain interaction (indicated with MPCs in Figure 21) is built between an hinged reference point and the metal connector, at the lateral surface of the threaded hole. The tensile load is then applied to the reference point that redistributes the force to the metal connector.

\footnotetext{
${ }^{18}$ Surface-to-surface interaction algorithm implemented in Abaqus [71], [72] is used. Friction-less interaction law is used. The master surface is on the contrast ring; the slave surface is on the glass.
} 


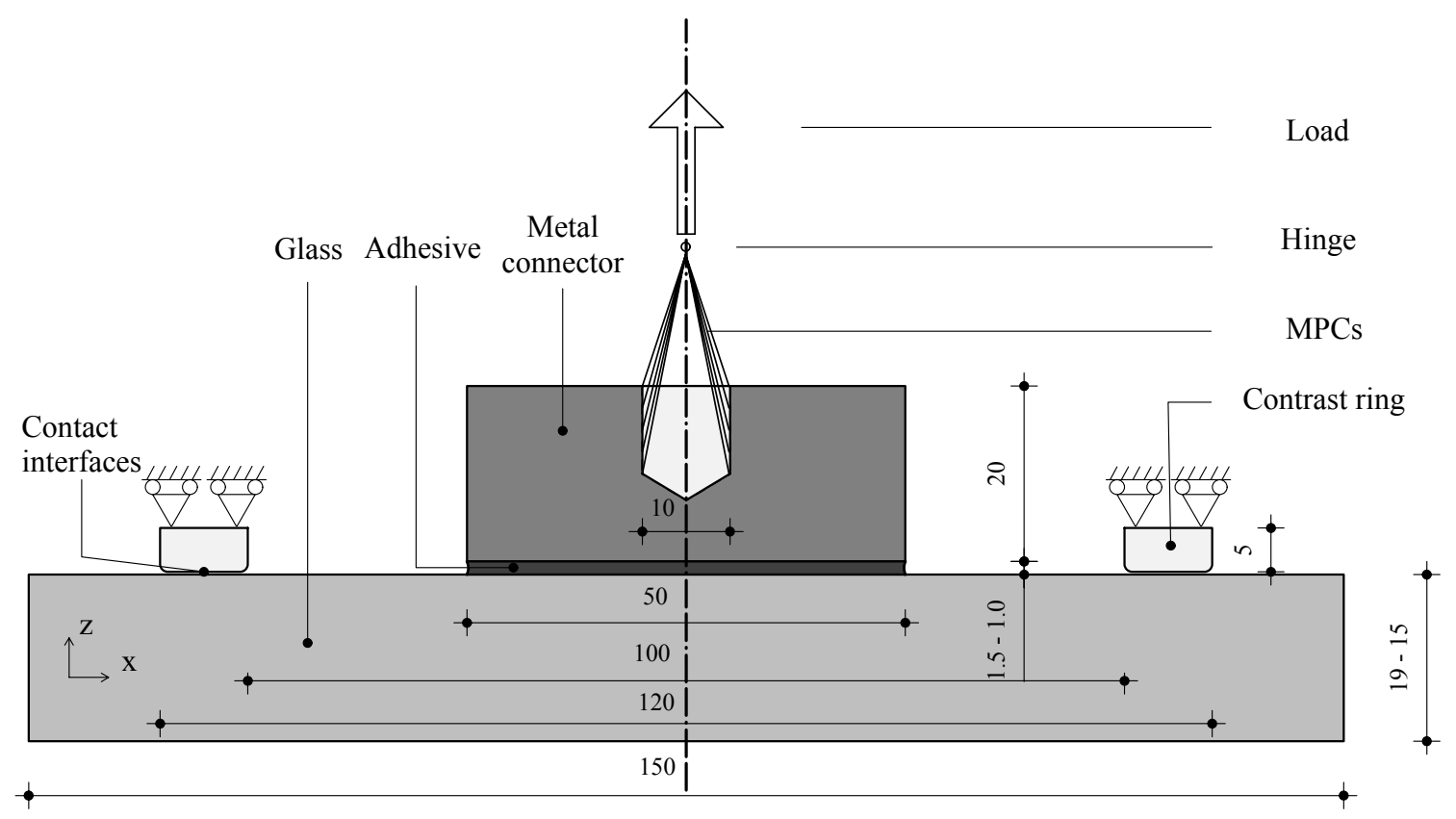

Figure 21: Geometry, dimension and boundary conditions of the numerical model

The results of mesh and elements study indicate triangular elements as being an optimal choice for this problem in terms of results accuracy and computational cost. The details of solver, element and mesh study can be found in [70]. Figure 22 shows the mesh of the model. The mesh is strongly refined at the level of the adhesive. Large stress gradient are indeed expected to occur in that region. For this reason, the mesh is also refined at the free edge of the adhesive itself (Figure 22 (b)). The element size at the adhesive is no larger than $0.2 \mathrm{~mm}$. Furthermore, the mesh size in the glass is also refined close to the contact region to facilitate the convergency of the contact analysis. Second order elements are used. Control of the pressure profiles is performed to ensure that volumetric locking is not occurring. All simulations show continuous pressure profiles. The use of elements with modified or hybrid formulation was therefore not required. Full precision is used because a large number of iterations is required to achieve the solution of the problem.

(a)

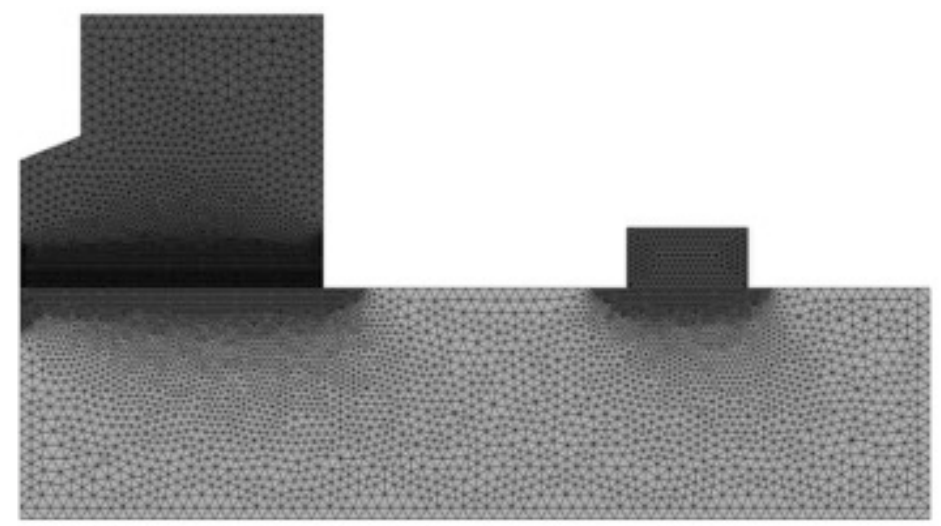

(b)

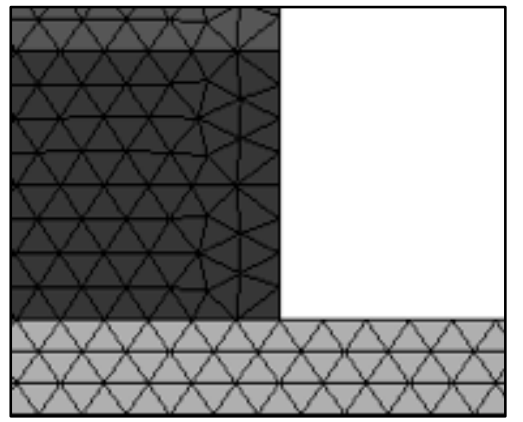

Figure 22: (a) Global view of the mesh of the numerical model of laminated connection under tensile force (c) close view at the adhesive perimeter

\subsection{Numerical results, parametrical study and discussion}

\subsubsection{Field distributions}


The stress field distributions in the adhesive of laminated connections under tensile load are now presented and discussed, firstly for TSSA and then for SG. The stress field distributions are extracted within the adhesive, at the interface between glass and adhesive, since the maximum stress peaks are occurring there. Given the complexity of the stress state of the adhesive, different types of stresses must be analysed to obtain a complete characterization of the adhesive stress state in the three dimensions. The following stresses are therefore computed:

- $\sigma_{n}$, stress along longitudinal direction (z-axis Figure 21), here called normal stress

- $\tau$, shear stress along radial direction, (x-axis Figure 21)

- $\sigma_{\max }$, maximum principal tensile stress

- $\sigma_{h}$, hydrostatic stress

- $q$, von Mises stress, also called equivalent stress

- $\theta$, hydrostatic angle.

The hydrostatic angle ${ }^{19}, \theta$, describes the hydrostatic level of the stress state, i.e. the ratio between hydrostatic and deviatoric components of the stress tensor. The angle $\theta$ is calculated as angle between the stress state and hydrostatic axis in a $q-\sigma_{h}$ plane. A full hydrostatic state is therefore described by $0^{\circ}$ hydrostatic angle, while a pure deviatoric stress state is described by a $90^{\circ}$ hydrostatic angle. The hydrostatic angle is the arctangent of the triaxiality, $\eta$, defined in literature as the ratio between hydrostatic pressure and equivalent stress.

The stress distributions are now presented in terms of normalized stress versus normalized distance. Normalized stresses, are defined as the actual stresses in the adhesive divided by the applied nominal stress, $\sigma_{\text {nom }}{ }^{20}$. The latter is calculated as the applied tensile force, $F_{N}$, divided by the surface area, $A$. Given the applied nominal stress, the normalized stresses describe the deviation of the non-linear distribution of the actual stress field from the uniform nominal stress distribution. Normalized distance is defined as the ratio between distance from the center and connection radius.

\footnotetext{
19 The hydrostatic angle is in this application preferred to the triaxiality because more stable in case of high hydrostatic stresses. The triaxiality varies from zero to infinity (fully hydrostatic state) while the hydrostatic angle varies from zero to $90^{\circ}$ (fully hydrostatic state).

${ }^{20}$ Notice that the stress distribution is calculated at load level close to the failure load to account for load effect. However, it should be notice that the load effect is observed to be rather limited. Indeed, simulation at different load levels (not here presented for the sake of brevity) have shown that load level effect induces variation between $1 \%$ and $4 \%$.
} 
(a)

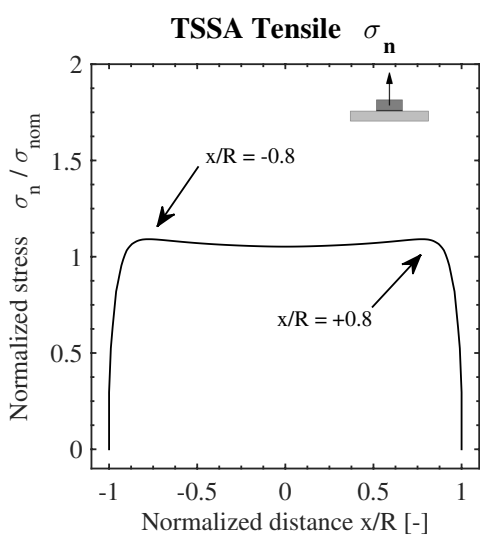

(d)

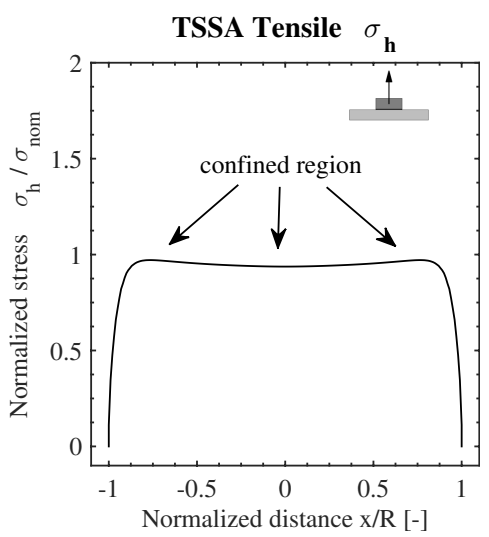

(b)

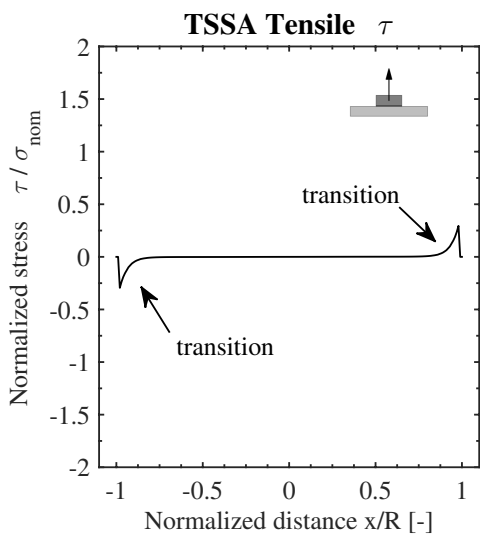

(e)

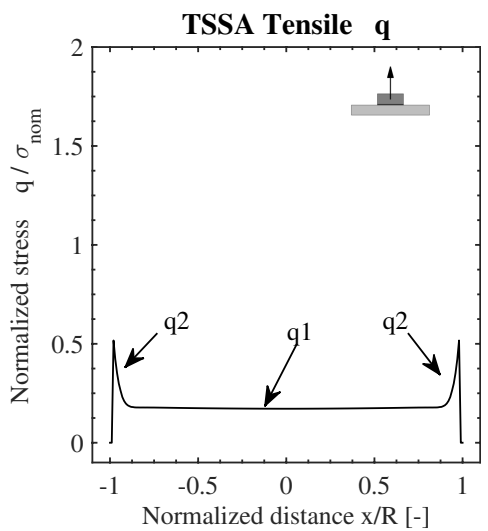

(c)

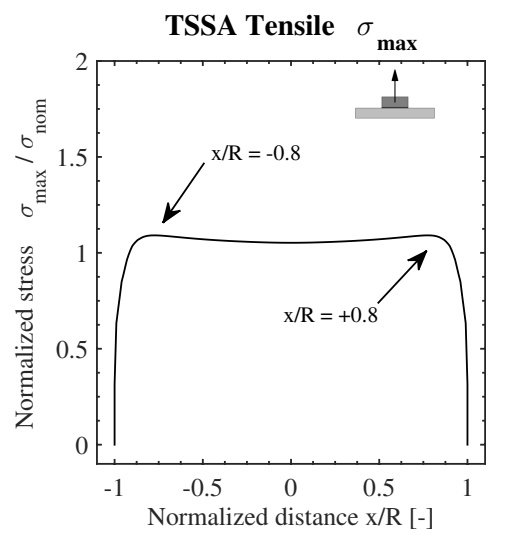

(f)

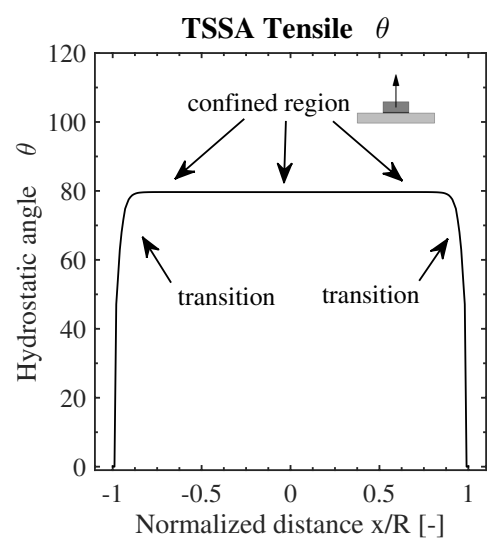

Figure 23: Stress field distribution in TSSA laminated connections under tensile force (a) normal stress (b) shear stress (c) maximum principal tensile stress (d) hydrostatic stress (d) equivalent stress (f) hydrostatic angle.

Figure 23 shows the stress distribution in the TSSA laminated connection under tensile force ${ }^{21}$. In general, it is observed that the stress state in the adhesive is rather complex. This is because, when a tensile load is applied to the connection, several mechanical phenomena are occurring in the adhesive. A detailed analysis is provided in the following paragraphs.

Figure 23 (a) shows that the normal stress distribution in the adhesive deviates from the uniform distribution and that shows it maximum value at around $0.8 \mathrm{R}$. The application of tensile load also develops shear stress close to the adhesive perimeter (Figure 23 (b)). This behaviour confirms the analytical consideration of the previous section. Indeed, close to the perimeter, shear stresses develop because of the transition between unconfined and confined state (see Figure 19). Once the confined region is reached (region a in Figure 19) the shear stress goes to zero. The principal tensile stress (see Figure 23 (c)) exhibits similar behaviour and magnitude as the normal stress, yet being slightly larger close to the perimeter due to the presence of shear stresses.

Figure 23 (d) shows then that the magnitude of the hydrostatic stress is large and close to the normal stresses. Hydrostatic stresses are developed for most of the adhesive region starting from the center up the above-mentioned transition region. Far from the perimeter, the three-dimensional stress tensor is thus dominated by its hydrostatic component. This indicates that large radial and tangential stresses are developed, confirming the hypothesis stated in the experimental and analytical study of the previous sections 4 and 5 .

\footnotetext{
${ }^{21}$ The TSSA normalized stress is temperature independent, since the modulus of elasticity and Poisson's ratio are stable with respect to temperature variation (see [44]).
} 
Figure 23 (e) indicates that the deviatoric component of the stress tensor is the sum of two contributions: a uniform distribution, $q_{1}$, and a stress peak at the perimeter, $q_{2}$. The first contribution is due to the difference between longitudinal and transversal stresses. This contribution is rather limited, yet not zero due to the difference between hydrostatic stress and normal stress ${ }^{22}$. The second contribution instead is due to the presence of shear stresses close to the perimeter, as schematically shown in Figure 19.

Finally, Figure 23 (f) shows the hydrostatic angle being in line with the above mentioned phenomena since it is (i) large in most of the adhesive due to the large hydrostatic component of the stress tensor caused by the confinement state and (ii) smaller in a transition region close to the perimeter.

(a)

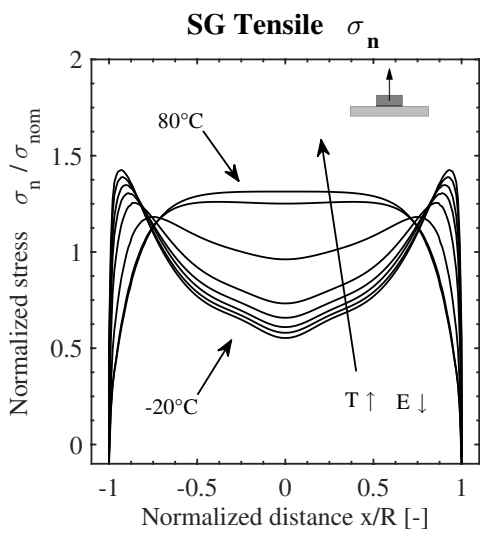

(d)

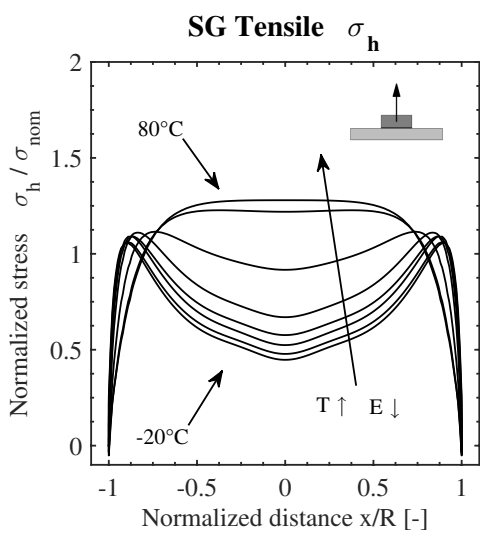

(b)

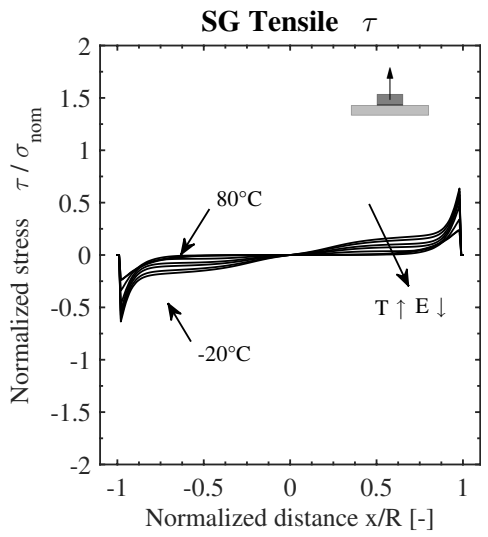

(e)

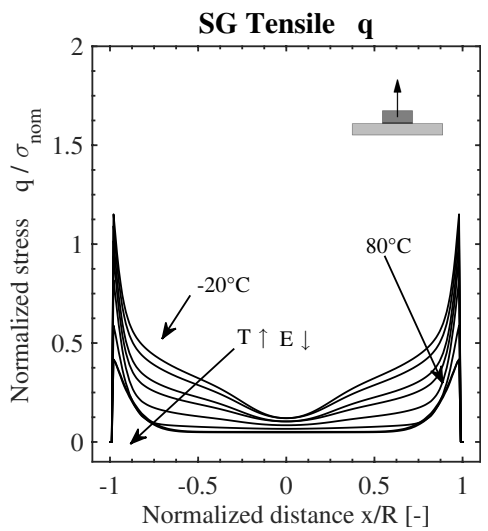

(c)

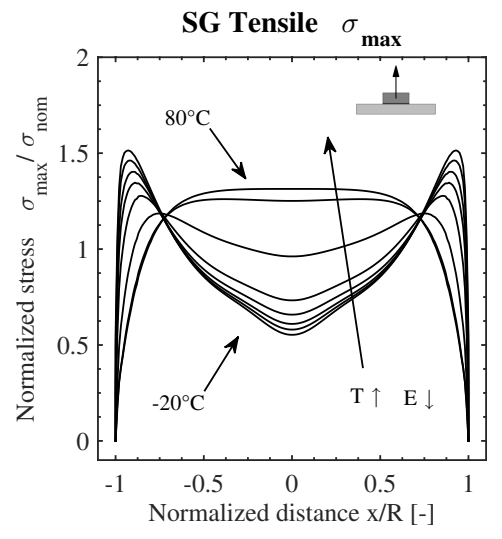

(f)

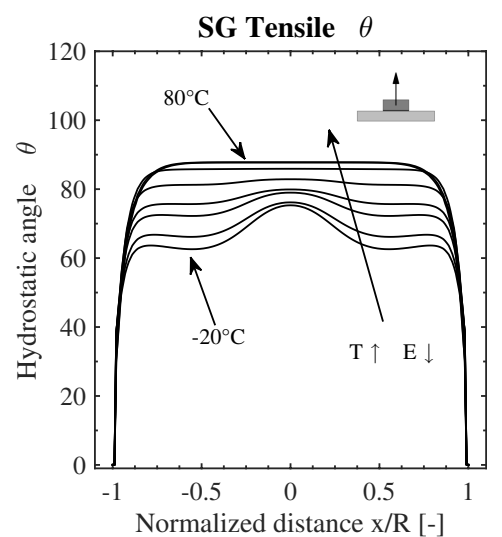

Figure 24: Stress field distribution in SG laminated connections under tensile force (a) normal stress (b) shear stress (c) maximum principal tensile stress (d) hydrostatic stress (d) equivalent stress (f) hydrostatic angle.

Figure 24 shows now the stress distributions in the SG laminated connection under tensile force, at different temperatures. As for TSSA, also the SG stress distributions are strongly non-linear and detailed analyses are required to interpret the mechanical phenomena occurring in the adhesive. In the following paragraphs the different three-dimensional stresses in the adhesive are discussed to provide a mechanical interpretation of the different phenomena.

In general, all considerations stated for the TSSA also apply to SG stress distributions. Similarly to TSSA: the field of the normal stress deviates from the uniform distribution, shear stresses develop at the transition region, large hydrostatic stresses develop far from the perimeter due to the confinement

\footnotetext{
22 The difference between hydrostatic and normal stresses is due to the difference between the TSSA Poisson's ratio and 0.5 , i.e. the condition of fully incompressible material.
} 
effect, deviatoric stresses are observed at the transition region close to the perimeter and a large hydrostatic angle is found in the central part of the adhesive.

In addition, since the mechanical properties of SG vary with temperature ${ }^{23}$, two main additional phenomena are occurring.

Firstly, at high temperatures, the adhesive material approaches the fully incompressible condition, since the Poisson's ratio tends to value of 0.5. Because of that, (i) the stresses redistribute towards the center part of the adhesive, where the response is stiffer than the perimeter due to the confinement effect (see Figure 24 and scheme of Figure 19) and (ii) the hydrostatic component of the stress tensor increases leading to large hydrostatic stresses, low deviatoric stresses and hydrostatic angle close to $90^{\circ}$ (see equation (7) in previous section, and hydrostatic stresses in Figure 24 (d) and the deviatoric stress of Figure $24(\mathrm{e}))$.

Secondly, at low temperatures, the deformability of the glass becomes comparable to the adhesive one. From this, two main consequences are observed: (i) additional shear stresses develop in the adhesive due to the glass bending and (ii) the vertical displacement field in the adhesive is non-uniform. More specifically, larger relative displacements between metal and glass are occurring at the perimeter (see following section). There, strain intensification occurs and consequently stress peaks migrate towards the perimeter (see Figure 24 (a)) ${ }^{24}$. In the following section, further analyses are performed to confirm this consideration on the effects of the adherend deformability.

\subsubsection{The effects of adherends deformability}

In the previous sections, analytical and numerical analysis of the SG stress distributions showed that, at low temperatures, the glass deformability has significant effects on the stress field distribution. In that regard, Figure 25 shows the distribution of the longitudinal displacement field in the adhesive: Figure 25 (a) in a soft adhesive (e.g. SG at $80^{\circ} \mathrm{C}$ ) and Figure 25 (b) in a rigid adhesive (e.g. SG at $20^{\circ} \mathrm{C}$ ). The longitudinal displacement field is uniform in soft adhesive while it is non linear in rigid adhesive, and strain intensifications are occurring close to the perimeter. This effect is due to the local bending of the glass and it develops when the compliance of the glass plate is comparable to the adhesive one. It is expected that this phenomenon has a non-negligible effect on the adhesive stress field distribution. Additional analyses are therefore performed to confirm this consideration. More specifically, two additional finite numerical analysis are carried out, implementing an infinitively rigid glass panel: laminated connections with $\mathrm{SG}$ at $80^{\circ} \mathrm{C}$ and with $\mathrm{SG}$ at $-20^{\circ} \mathrm{C}$. The results of these analyses are then compared with the stress distributions obtained modelling the actual deformability of the glass adherend (see Figure 26). In the plots of Figure 26, dashed lines represent results with infinitively rigid glass panel while continuous lines indicate results with the actual glass deformability.

\footnotetext{
${ }^{23}$ Going from -20 to $+80^{\circ} \mathrm{C}$ the SG modulus of elasticity varies from approx. $1000 \mathrm{MPa}$ to $3 \mathrm{MPa}$ and the Poisson's ratio from 0.41 to 0.49 (see [44])

${ }^{24}$ The stress peak is still slightly away from the perimeter, even if the maximum normal strain is at the edge. This is because at the perimeter the apparent stiffness is lower then far from it. Indeed, with same applied displacement, stiffer materials develop larger stress than softer ones. In this case, the stiffer material is far from perimeter so with uniform deformation the maximum stress is expected to occur in the central part of the adhesive. Therefore the stress distribution is the results of two effects: increasing strain with max at the edge (that would lead stress peak at the edge in case of constant stiffness) and reducing stiffness going towards the perimeter. This leads to an intermediate behaviour with a peak slightly close yet not at the perimeter. This explains also the location of the stress peak in the TSSA.
} 
(a)
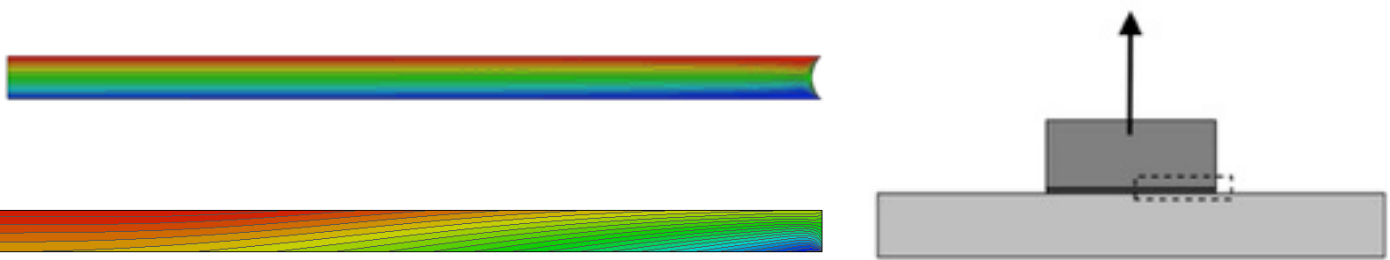

(b)

Figure 25: Distribution of the displacement field and deformation along z-direction of the adhesive from middle to perimeter (left to right) (a) soft adhesive (b) rigid adhesive

Firstly, all plots in Figure 26 show that at $80^{\circ} \mathrm{C}$ the glass deformability has minor effect, since dashed and continuous lines are overlapping. At $-20^{\circ} \mathrm{C}$ instead, the two analyses provide different results. Firstly, Figure 26 (a) shows that a model with infinitively rigid glass panel indicates maximum values of normal stress located in the centre of the adhesive rather than close to the perimeter. Secondly, Figure 26 (b) shows that with rigid adherend the deviatoric component of the stress tensor is limited ${ }^{25}$. Thirdly, Figure 26 (c) shows that, with rigid adherend, the distribution of the hydrostatic angle is uniform, while with actual glass deformability a non-uniform distribution is obtained.

It is therefore concluded that the considerations stated in the previous section on the adherend deformability are confirmed. More in detail, in the case of a stiff adhesive, the hypothesis of rigid glass adherend leads to non-negligible deviation from the real mechanical response of the connection. The compliance of the glass panel must thus be accurately modelled for a correct computation of the adhesive stress field distribution.

(a)

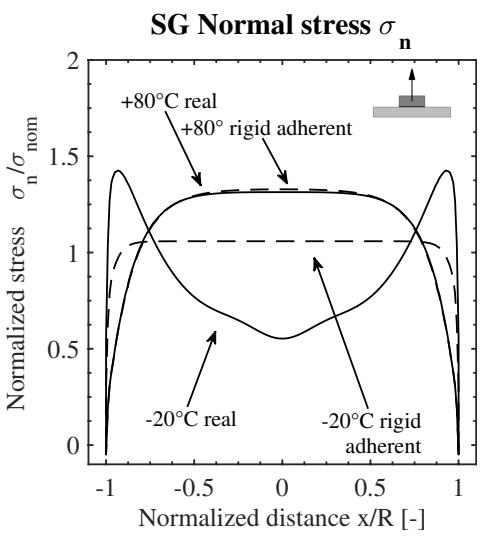

(b)

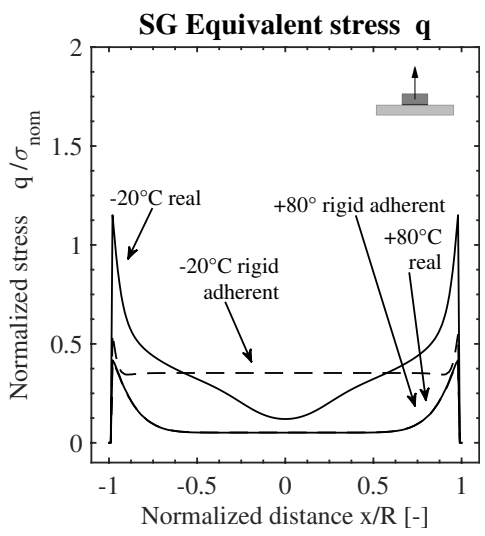

(c)

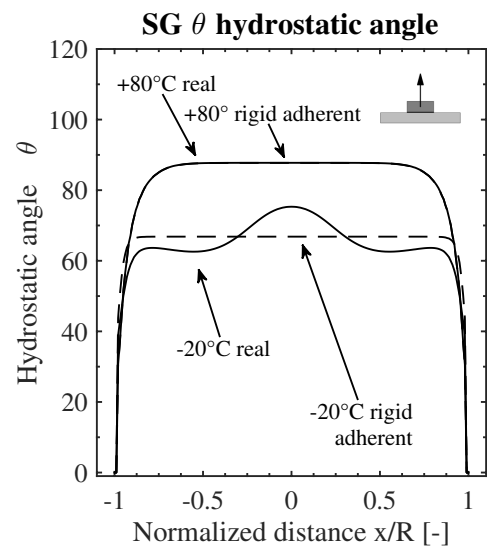

Figure 26: Effect of the adherend deformability on the stress distribution of SG connection at high and low temperatures. Dashed lines are result of simulation with infinite rigid adherends, continuous lines are results with real adherend compliance (a) normal stress (b) equivalent stress (c) hydrostatic angle

\subsubsection{Comparison with experimental results}

In this section the results of the analytical and numerical analyses are compared to the experimental observations. The aim is to confirm the considerations made in the analytical study and to validate the mechanical phenomena predicted by the numerical model. More in detail, this comparison focuses on:

- The TSSA stress field distribution and the location of the maximum peak

- The TSSA whitening following the stress field distribution of the hydrostatic component of the stress tensor

\footnotetext{
${ }^{25}$ With rigid adherend the deviatoric field distribution indicates shear stresses mainly located at the transition region close to the perimeter, while with deformable adherend additional shear stresses develop also across the adhesive area.
} 
- The presence of a transition region close to the perimeter of the TSSA adhesive with reduced hydrostatic stresses

- The location and failure mode of SG failure at room and low temperature

- The location and evolution of bubble formation in the SG at high temperature

- The presence of a transition region close to the perimeter of the SG adhesive with reduced hydrostatic stresses

Figure 27 (a) shows the hydrostatic stress distribution in a TSSA connection computed by the numerical model. Two circles indicate the locations of the maximum values. The maximum hydrostatic stress is occurring at approximately $0.8 \mathrm{R}$. According to the hypothesis of the whitening phenomenon being related to the hydrostatic component of the stress tensor, the whitening is expected to first occur in a small ring between the center and the perimeter of the adhesive (i.e. radius about $0.8 \mathrm{R}$ (see scheme of Figure 27 (b)). The experimental observations confirm the model prediction since the whitening is occurring first in this region (see Figure 27 (c)), similar to the indication provided by the numerical model. Since this is happening at any temperature, this also indicates that the stress field distribution is not affected by temperature variation. An additional source of validation is provided by the failure location observed after testing. As for the whitening, also the location of failure (see localized point of fracture in Figure 28) is in agreement with the prediction provided by the model. Indeed, the failure location is located in a region between the center and the perimeter of the adhesive at approximately $0.8 \mathrm{R}$, in agreement with the results plotted in Figure 27 (a).

(a)

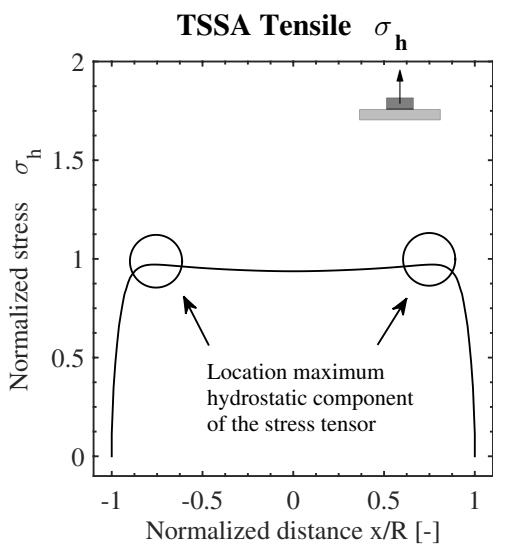

(b)

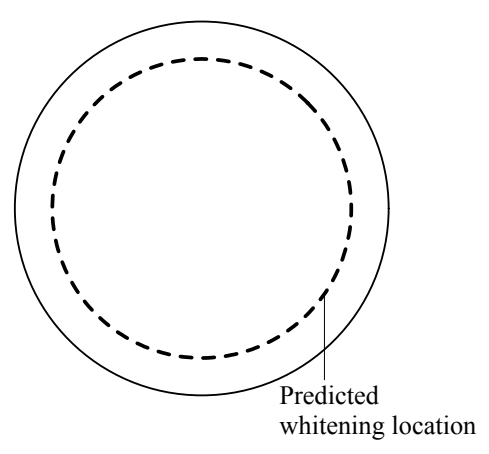

(c)

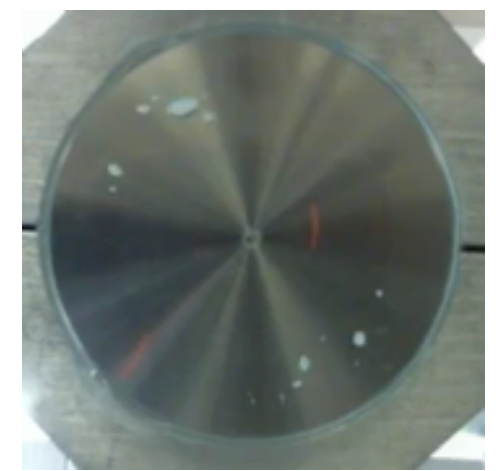

Figure 27: Comparison between model and experimental observation of the starting of whitening phenomenon

(a)

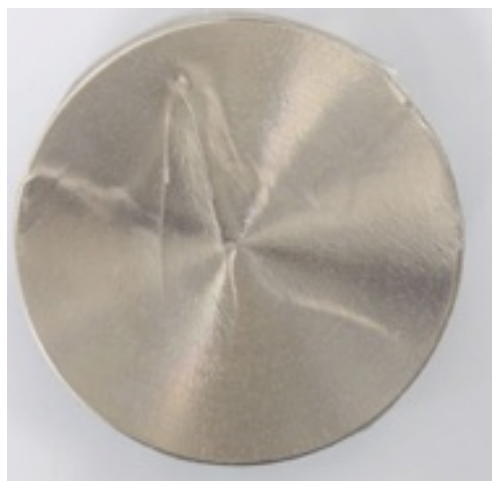

(b)

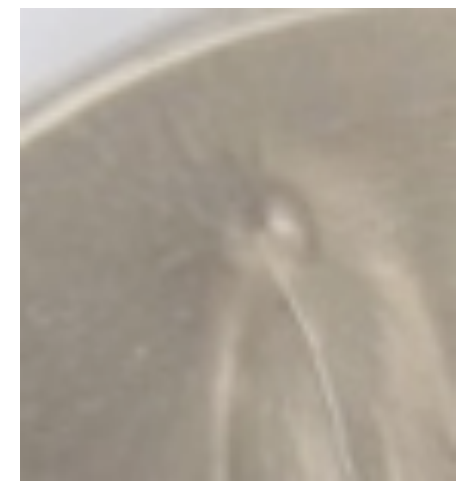

Figure 28: (a) TSSA laminated connection after tensile test (b) close view at the failure location

Figure 29 (a) shows the distribution of the hydrostatic angle in TSSA connection, computed by the numerical model. The model indicates a dominating deviatoric component of the stress tensor for most of the adhesive area. Close to the perimeter, instead, the hydrostatic angle is smaller than in the center, 
which indicates smaller ratio between hydrostatic and deviatoric component. It is therefore expected to observe less or no whitening close to the perimeter. The behaviour given by the model is confirmed by the experimental observation (Figure 29 (b)). Indeed, during the test, a small ring of 1-2mm with no whitening is observed at the transition region, which confirms that there the stress tensor has a less dominant hydrostatic component.

(a)

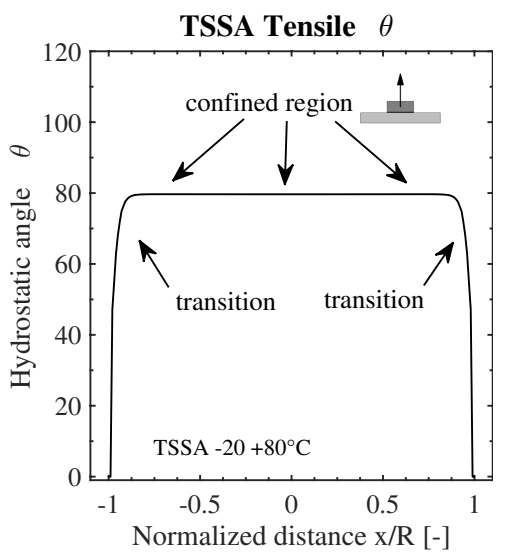

(b)

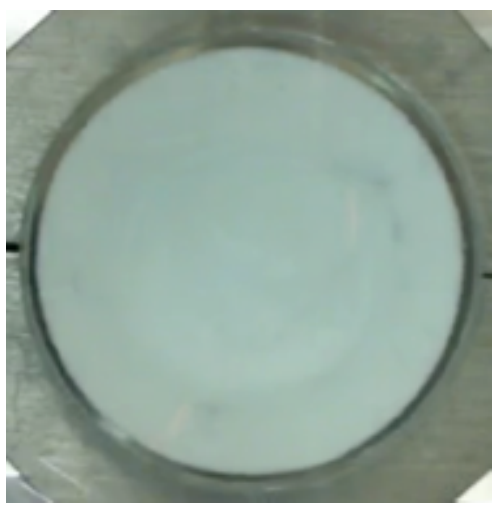

Figure 29: Comparison between model and experimental observation of the transparent ring of adhesive close to the perimeter

Figure 30 (a) shows the field distribution of the equivalent stress in the SG laminated connections at room and low temperatures (for the sake of clarity deformations are amplified 10x). The results of the model indicate large deviatoric stresses occurring close to the perimeter of the adhesive. As discussed above, this is due to the transition between perimeter and confined region. Figure 30 (b) shows a photo of a typical SG laminated connection after failure at room temperature. The failure is usually located close to the perimeter, showing an inclined failure plane through thickness, similar to the stress field distribution of Figure 30 (a).

(a)

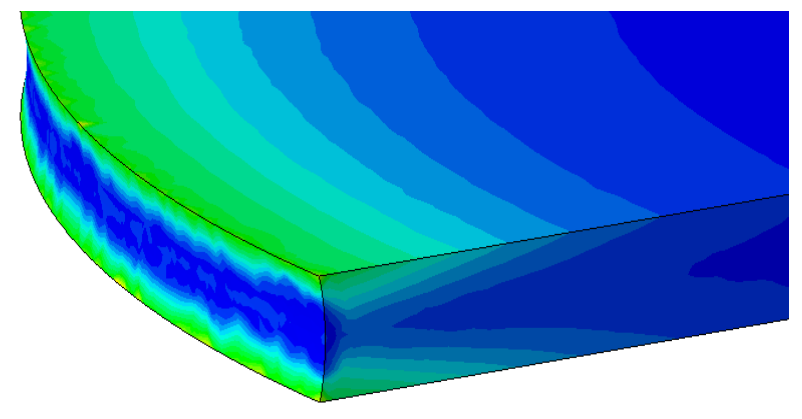

(b)

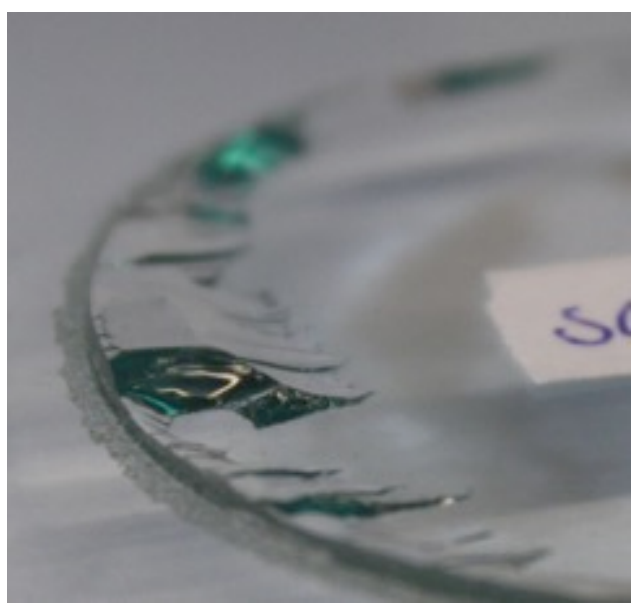

Figure 30: Comparison between results of the finite element numerical modelling and the experimental observation (a) stress field distribution computed by FEM analysis, (b) SG laminated connection failure surface after testing at low temperature

Figure 31 (a) shows the hydrostatic stress distribution in SG connections at high temperature computed by the numerical model. The region of the maximum hydrostatic stress is indicated by a horizontal arrow, i.e. in the center of the connection. There the adhesive is subjected to large confinement state and therefore transversal stresses develop. At high temperature, given a Poisson's ratio close to 0.5 , the bubbles are expected to appear in a central region of the adhesive (see scheme of 
Figure 31 (b)), in agreement with the hypothesis of bubbles phenomenon being related to a dominant hydrostatic component of the stress tensor. The experimental observations confirm the model prediction. The bubbles are occurring in the middle of the adhesive, over a region far from the perimeter (see Figure 31 (c)).

(a)

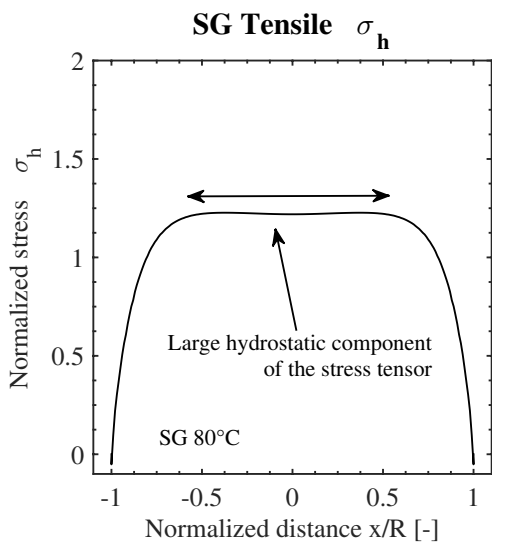

(b)

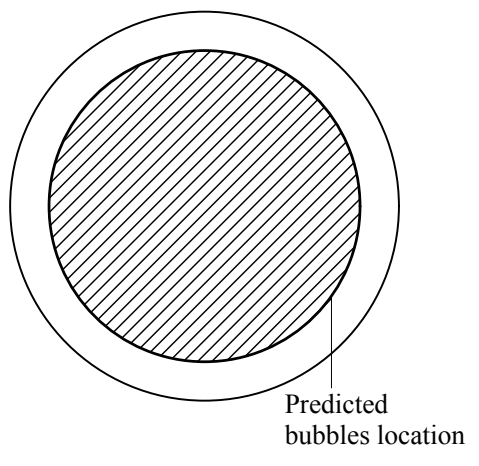

(c)

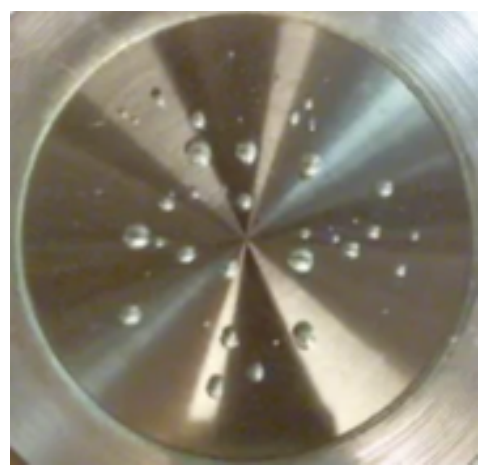

Figure 31: Comparison between model and experimental observation of the bubbles phenomenon

Figure 32 (a) shows the distribution of the hydrostatic angle in SG connection computed by the numerical model. The model indicates small deviatoric component of the stress tensor for most of the adhesive area. Close to the perimeter, instead, at the transition region between perimeter and the fully confined region, the hydrostatic angle reduces. This indicates a smaller ratio between hydrostatic and deviatoric component of the stress tensor. According to the model predictions, it is therefore expected to observe less or no bubbles close to the perimeter. The indications given by the model are confirmed by the experimental observation (Figure 32 (b)). Indeed, during the test, a small ring of approx. $2 \mathrm{~mm}$ with no bubbles is observed at the transition region, indicating a stress tensor with less dominant hydrostatic component.

(a)

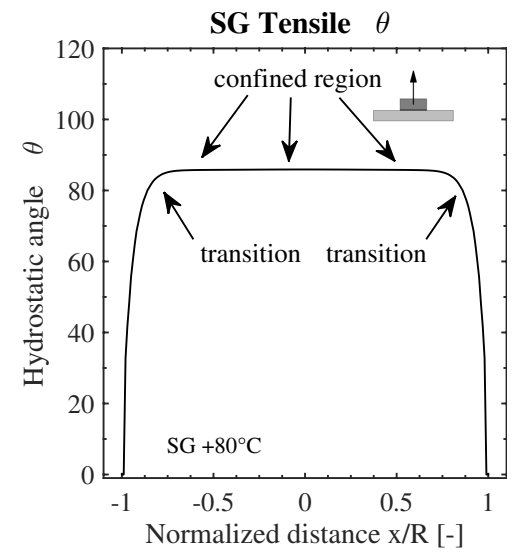

(b)

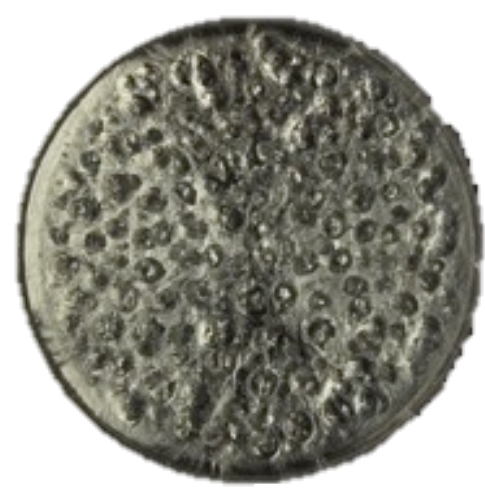

Figure 32: Comparison between model and experimental observation of the adhesive ring free of bubble close to the perimeter

An additional consideration can be made on the bubble phenomenon. In the experiments, bubbles occur approximately equidistantly between each other (see Figure 33 (b)). This phenomenon confirms the mechanical consideration stated above on the major role played by the hydrostatic stress. Indeed, when a bubble is formed, the surface of the bubble represents a stress-free surface. At this surface, the adhesive is not fully confined and the stresses normal to the surface are equal to zero. A new bubble 
cannot therefore develop close to an existing bubble. A certain distance is instead needed to develop back the transversal stresses. Moving away from the bubble surface, transversal stresses develop ${ }^{26}$, and a new bubble is formed only when enough hydrostatic pressure is developed (Figure 33 (a)). Given the spherical nature of the bubbles, this phenomenon is occurring along both radial and tangential direction. This explains the uniform distance between bubbles in both the tangential and radial direction.

(a)

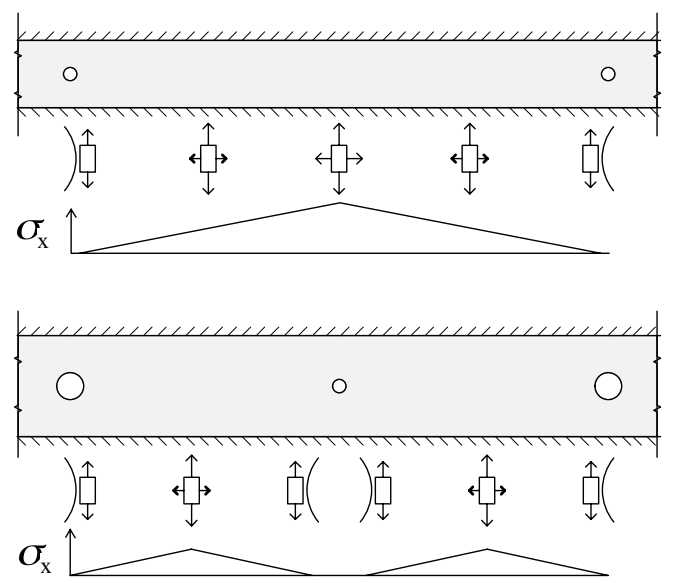

(b)

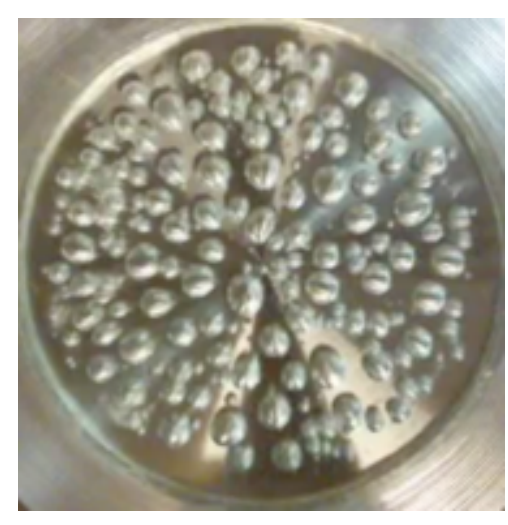

Figure 33: (a) Scheme of stress state between bubbles (b) Photo of SG laminated connection under tensile force at high temperature

\footnotetext{
${ }^{26}$ This is similar to the phenomenon happening in the transition region between the confined region and the perimeter of the adhesive.
} 


\subsubsection{Validation with experimental results using different geometries}

In this section, additional analyses of a laminated connection with different geometries are performed with the goal to provide an additional validation of the modelling results. Both radius of the metal connector and adhesive thickness are investigated. The results are then compared with the experimental investigation performed in [21], [26]. The following geometries are investigated.

- Radius of $25 \mathrm{~mm}$, adhesive thickness of $3 \mathrm{~mm}$

- Radius of $80 \mathrm{~mm}$, adhesive thickness of $3 \mathrm{~mm}$

- Radius of $80 \mathrm{~mm}$, adhesive thickness of $1 \mathrm{~mm}$

- Radius of $10 \mathrm{~mm}$, adhesive thickness of $1 \mathrm{~mm}$

Mesh, element and boundary conditions ${ }^{27}$ are as described in the previous section for the other finite element simulation.

Figure 34 (a) shows the field distribution of the hydrostatic stresses in $25 \mathrm{~mm}$ radius connection with adhesive thicknesses of $1 \mathrm{~mm}$ (dashed line) and $3 \mathrm{~mm}$ (continuous line). The $1 \mathrm{~mm}$ case is similar to the one already discussed previously in this work. The $3 \mathrm{~mm}$ case is the one tested in [21], [26]. For the $3 \mathrm{~mm}$ connection, the numerical model gives maximum values located in the middle of the connection. The numerical results are confirmed by the experimental observation of [21], [26]. Indeed, in the case of $\mathrm{t}=3 \mathrm{~mm}$ the whitening starts in the centre of the connection (see Figure 34 (b)) as indicated by the numerical model.

(a)

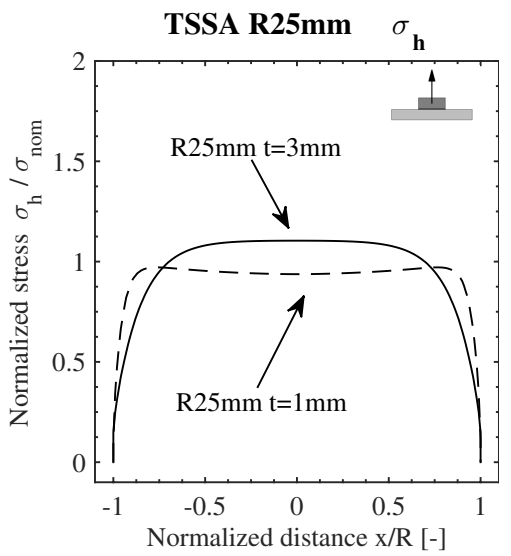

(b)

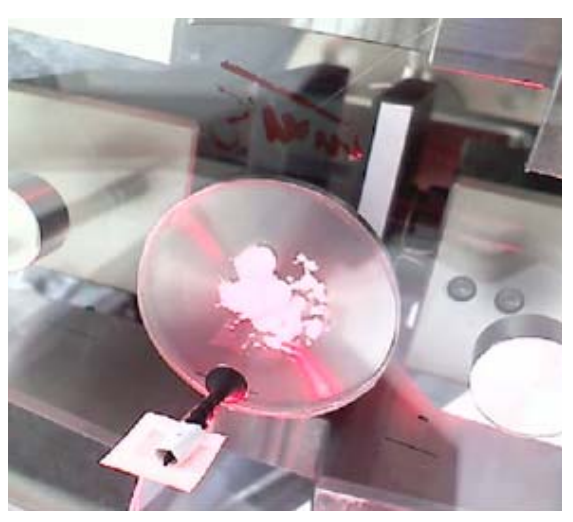

Figure 34: (a) Stress field distribution in a 50mm TSSA laminated connection: comparison between $1 \mathrm{~mm}$ and $3 \mathrm{~mm}$ adhesive thickness (b) whitening phenomenon in a $3 \mathrm{~mm}$ TSSA laminated connection located in the center region of the connection

Figure 35 (a) shows the field distribution of the hydrostatic stresses in a 40mm radius connection with adhesive thicknesses of $1 \mathrm{~mm}$ (dashed line) and $3 \mathrm{~mm}$ (continuous line). The numerical model gives maximum values located in the centre region of the connection with the $3 \mathrm{~mm}$ adhesive, while with $1 \mathrm{~mm}$ adhesive the maximum values migrates towards the perimeter. The numerical results are validated by the experimental observation of [21], [26]. In the case of $3 \mathrm{~mm}$ adhesive the whitening start in the centre while in the case of $1 \mathrm{~mm}$ adhesive the whitening starts close to the perimeter (see Figure 35 (b) versus Figure 35 (c))

\footnotetext{
${ }^{27}$ It should be notices that the boundary conditions were not axial symmetric and the distance to the support was not given. In this sections similar conditions to the previous analysis are assumed. Further analysis might be therefore required.
} 
(a)

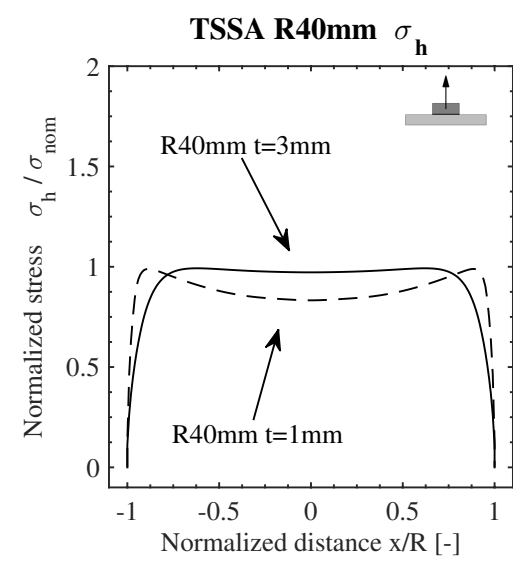

(b)

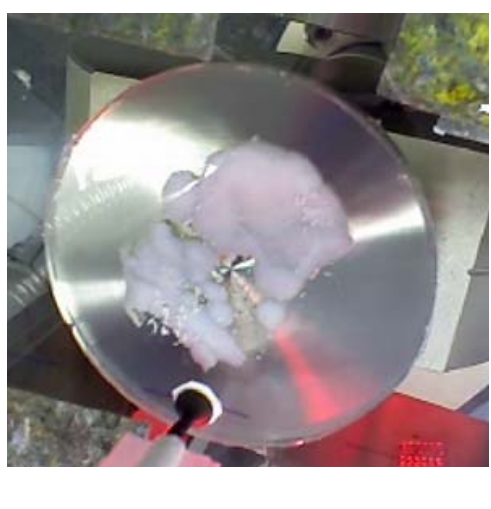

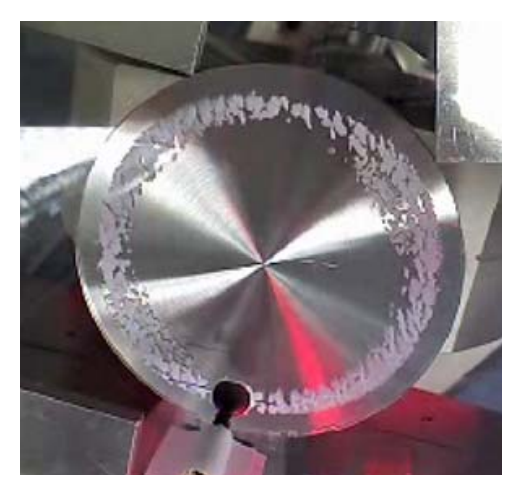

Figure 35: (a) Stress field distribution in a 80mm TSSA laminated connection: comparison between $1 \mathrm{~mm}$ and $3 \mathrm{~mm}$ adhesive thickness (b) whitening phenomenon in a 3mm TSSA laminated connection located in the center region of the connection (c) whitening phenomenon in a $1 \mathrm{~mm}$ TSSA laminated connection located in a region close to the perimeter of the connection

Figure 36 (a) shows the field distribution of the hydrostatic stresses in a $10 \mathrm{~mm}$ radius connection and adhesive thicknesses of $1 \mathrm{~mm}$ (continuous line). The $1 \mathrm{~mm}$ case is similar to the one already discussed in this work. The $3 \mathrm{~mm}$ case is tested in [21], [26]. In this latter case the numerical model gives maximum values located in the middle of the connection. The numerical results are validated by the experimental observation of [21], [26]. In the case of $t=3 \mathrm{~mm}$ the whitening starts in the centre of the connection (see Figure 36 (b)).

(a)

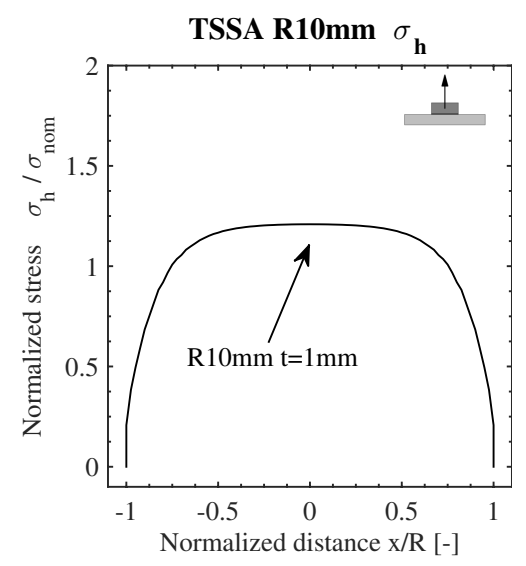

(b)

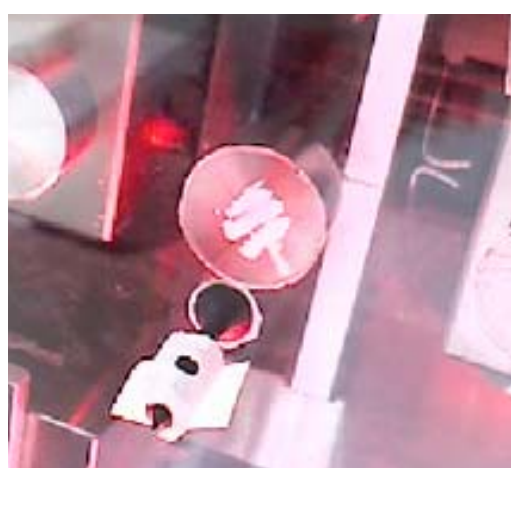

Figure 36: (a) Stress field distribution in a 20mm TSSA laminated connection with $1 \mathrm{~mm}$ adhesive thickness (b) whitening phenomenon located in the center region of the connection

In conclusion, it is shown that the results of the additional numerical analysis performed on different geometries are validated by the experimental campaign of [21], [26]. It is observed that the diameterthickness ratio plays an important role in the mechanical response of the connection. This is because with large $\mathrm{D} / \mathrm{t}$ ratio, the confinement effect is large, the confined stiffness increases and therefore the maximum stress location tends to migrate towards the perimeter. Indeed, if compared to the other cases, when the $\mathrm{D} / \mathrm{t}$ ratio is smaller (either with a $3 \mathrm{~mm}$ adhesive or with $10 \mathrm{~mm}$ radius), the experimental results show a migration of the maximum stress towards the center, in line with the analytical and numerical results discussed in previous sections. 


\subsubsection{Stress factors for TSSA and SG laminated}

In the previous sections, finite numerical models are used to investigate in detail the mechanical behaviour of laminated connections and to confirm the analytical considerations of section 5 . More specifically, non-linear numerical analyses are performed to compute the three-dimensional stress state in the adhesive and to quantify the stress peak occurring in the adhesive at different temperatures. A full description of the stress state is given, since all components of the stress tensor are computed and discussed. In this section, these results are summarized by means of a set of non-dimensional factors, here called stress factors. Given the applied nominal stress, the aim of the stress factor is to compute the peak values of each key stress component that is necessary to fully describe the stress state in the adhesive.

Stress factors are defined as the ratio between the actual stress peaks computed by the non-linear finite element analysis and the nominal stress. More specifically, two stress factors are of main interest: the normal stress factor, $\alpha_{N}$ and the hydrostatic stress factor $\alpha_{h}$, here defined by the first two terms of equation (15). The use of these stress factors, together with the hydrostatic angle, synthesizes the results of the previous section yet gives a full picture of the adhesive stress state. Namely, given the applied nominal stress, it is possible to compute the stress peak in the normal stress distribution, the hydrostatic component of the stress tensor and the triaxiality in the adhesive at different temperature. In addition, using the hydrostatic stress factor with the hydrostatic angle, the deviatoric stress factor can be calculated.

$$
\alpha_{n}=\frac{\sigma_{n}}{\sigma_{\text {nom }}} ; \quad \alpha_{h}=\frac{\sigma_{h}}{\sigma_{\text {nom }}} ; \theta=\operatorname{atan}(\eta) ; \alpha_{q}=\frac{q}{\sigma_{\text {nom }}} ;
$$

The use of these stress factors together with the results of the experimental investigations allows the derivation of a failure prediction model, which is described more in detail in the following section. Table 8 collects the stress factors computed for TSSA and SG at different temperatures. The SG stress factors are plotted as a function of the temperature in Figure 37. The TSSA stress factors are temperature independent.

Figure 37 (a) shows the behaviour of normal, hydrostatic and deviatoric stress factors of SG as a function of the temperature. Going from a low to a high temperature, the normal stress factor firstly decreases and then increases above $40^{\circ} \mathrm{C}$. This behaviour results from the combination of the different non-linear phenomena described in the previous sections, such as confined stiffness and adherend deformability. The hydrostatic stress factor is large and increases at high temperature because of the Poisson's ratio effect. The deviatoric stress factor is smaller than the other two, with a larger value at low temperature. This is due to the difference between hydrostatic stress and nominal stress and due to adherend deformability. Figure 37 (b) shows the hydrostatic angle at different temperatures. The dotted line represents the hydrostatic angle of a theoretically fully incompressible material in a fully confined state. The continuous line instead represents the actual hydrostatic angle. The hydrostatic angle is close to $90^{\circ}$ at high temperatures because Poisson's ratio tends to 0.5 and is lower at low temperatures due to the lower Poisson's ratio and the reduced triaxiality of the stress tensor. Finally, Figure 37 (c) shows how the confinement effect (i.e. development of transversal stresses when longitudinal stresses are applied) changes with temperature. The ratio between hydrostatic and normal stress obtained from FEM analysis is plotted as a function of the temperature with a continuous line. Dashed line represents instead the results of the analytical calculation according to equation (7). Numerical and analytical predictions appear to be in good agreement ${ }^{28}$. Figure 37 (c) shows that at low

\footnotetext{
${ }^{28}$ The deviation between the analytical and numerical results at room and low temperatures is due to the hypothesis of fully confinement state $\left(\varepsilon_{x}=0\right)$ that fully applies only far from the edge. When the maximum stress migrates towards the perimeter, as it happens at room and low temperatures (see Figure 24 (a)), non-zero transversal radial deformation develops and the confinement effects reduces. Note that the ratio between the hydrostatic and normal stresses does not necessary coincide with the hydrostatic angle. The latter indeed accounts also for the deviatoric component of the stress tensor, which is the sum of two contributions $q_{1}$ and $q_{2}$, described in detail in the previous section.
} 
temperature the confinement effect on the stress tensor is lower than at high temperature. More specifically at $-20^{\circ} \mathrm{C}$ the hydrostatic stress is equal to $76 \%$ of the normal stress while at $80^{\circ} \mathrm{C}$ the hydrostatic stress is equal to $97 \%$ of the nominal stress.

(a)

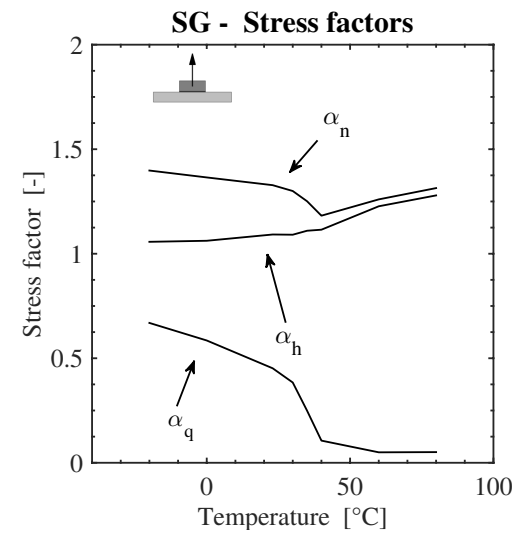

(b)

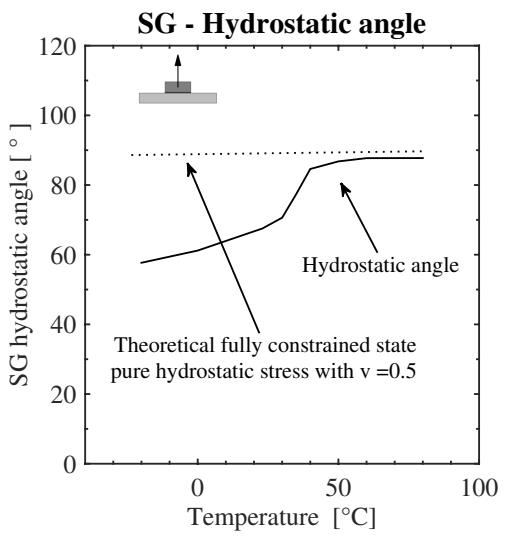

(c)

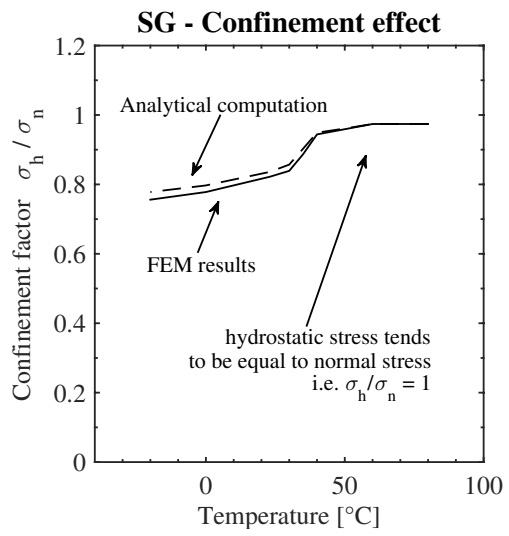

Figure 37: Plots of SG stress factors at different temperatures

Table 8: Stress factors for TSSA and SG laminated connection under tensile force

\begin{tabular}{cccccccc}
\hline Temperature & Material & $\alpha_{n}$ & $\alpha_{h}$ & $\theta$ & $\alpha_{q}$ & $\alpha_{h} / \alpha_{n}$ \\
\hline $80^{\circ} \mathrm{C}$ & $\mathrm{SG}$ & 1.31 & 1.28 & 87.74 & 0.05 & 0.97 \\
$60^{\circ} \mathrm{C}$ & $\mathrm{SG}$ & 1.26 & 1.23 & 87.72 & 0.05 & 0.97 \\
$50^{\circ} \mathrm{C}$ & $\mathrm{SG}$ & 1.22 & 1.17 & 86.78 & 0.08 & 0.96 \\
$40^{\circ} \mathrm{C}$ & $\mathrm{SG}$ & 1.18 & 1.12 & 84.61 & 0.11 & 0.94 \\
$30^{\circ} \mathrm{C}$ & $\mathrm{SG}$ & 1.30 & 1.09 & 70.62 & 0.38 & 0.84 \\
$23^{\circ} \mathrm{C}$ & $\mathrm{SG}$ & 1.33 & 1.09 & 67.53 & 0.45 & 0.82 \\
$0^{\circ} \mathrm{C}$ & $\mathrm{SG}$ & 1.36 & 1.06 & 61.19 & 0.59 & 0.78 \\
$-20^{\circ} \mathrm{C}$ & $\mathrm{SG}$ & 1.40 & 1.06 & 57.69 & 0.67 & 0.76 \\
$-20^{\circ} \mathrm{C}+80^{\circ} \mathrm{C}$ & $\mathrm{TSSA}$ & 1.09 & 0.97 & 79.53 & 0.18 & 0.89 \\
\hline
\end{tabular}




\section{Failure prediction model}

A general failure model that accounts for the non-linear stress distribution, boundary conditions, geometry, temperature dependent behaviour and strain rate dependent behaviour is here proposed by the equation (16). In equation (16), $F_{N}[\mathrm{kN}]$ is the tensile resistance, $A\left[\mathrm{~mm}^{2}\right]$ is the adhesive surface of the laminated connection, $f_{N}[\mathrm{MPa}]$ represents the reference tensile resistance that is equal to $6.00 \mathrm{MPa}$ for TSSA and 18.76 for SG, $\alpha_{n}[-]$ is the stress factor defined in the previous paragraph, $\alpha_{\varepsilon}[-]$ is a normalized factor that accounts for strain rate and $\alpha_{T}[-]$ is a normalized factor that accounts for the temperature. In $\alpha_{\varepsilon}$ and $\alpha_{T}$, strain rate values are in [1/sec] and temperature values are in $\left[{ }^{\circ} \mathrm{C}\right]$.

$$
F_{N}=f_{N} \cdot \frac{1}{\alpha_{n}} \cdot \alpha_{\varepsilon} \cdot \alpha_{T} \cdot A
$$

Non-linear Matlab scripts are developed for the derivation of the model following a probabilistic approach. The scripts are developed to allow the derivation of (i) a model defined over one or more dimensions (in this case the two dimensions are strain rate and temperature) (ii) a model with a variable standard deviation and (iii) a model dependent on one or more parameters. The latter are described in detail in the following two sections for TSSA and SG. The model is assumed to follow a normal probability density function (pdf). However, different pdfs can be also implemented. The objective function of joint probability is then maximized within constrain functions.

Table 9 shows a first comparison between models and experimental results for both adhesives by means of the coefficient of determination, $R^{2}$, the mean and covariance of the model-test ratio. The following two sections compare in more detail the tensile resistance given by the models to the experimental observations of TSSA and SG laminated connections under tensile loading. Firstly, the model is directly compared with experiments with one-dimensional plots. Secondly, the ratio between model and experiments is calculated and plotted for all investigated configurations of strain rate and temperature. These ratio plots in normalized scale allow to evaluate the accuracy of the model (deviation from unity) that might be not sufficiently clear in an absolute scale for all investigated test configurations. Thirdly, the residuals, i.e. difference between model and test results are calculated. This is done to analyse the variance of the model and namely to assess that the residuals have a random behaviour and tend to a normal distribution.

It should be noted that the proposed models are to be used only within the investigated range of strain rates, temperatures and triaxiality. Further analyses must be performed to extend the model out of the investigated ranges.

Table 9: Summary of models-experimental comparisons

\begin{tabular}{|c|c|c|c|}
\hline \multirow{2}{*}{ Model } & \multicolumn{2}{|c|}{$F_{\text {model }} / F_{\text {test }}$} & \multirow{2}{*}{$\mathrm{R}^{2}$} \\
\hline & Mean & $\mathrm{Cov}$ & \\
\hline TSSA & 0.9951 & 0.0362 & 0.8695 \\
\hline SG & 0.9855 & 0.1790 & 0.8006 \\
\hline
\end{tabular}

\subsection{Model for TSSA laminated connections under tensile load}

In this section the tensile resistances given by the TSSA model are compared with the experimental results. The residual, calculated as the difference between model and experimental observations, are also plotted. The strain rate factor proposed for the TSSA model is given by equation (17). The temperature factor proposed for the TSSA model is given by equation (18). More specifically, a logarithmic law is proposed for the effects of strain rate variation and linear law is instead proposed for the effects of temperature variation. 


$$
\begin{aligned}
& \alpha_{\varepsilon, T S S A}=1.21+\frac{\log (\dot{\varepsilon})}{15.71} \\
& \alpha_{T, T S S A}=1.02-\frac{T}{398.45}
\end{aligned}
$$

It is observed that the experimental results follow the proposed TSSA model for the full range of investigated temperatures (see Figure 38 (a) and Figure 39(a)). The experimental results also follow the proposed TSSA model for the strain rate variation (see Figure 38 (b) Figure 39 (b)). The deviation between model and test results is small for the temperature variation. The residual are larger with data at different strain rates (see last 6 datum in Figure 38 (c)). A quadratic order equation or a more complex expression for the strain rate coefficient might be proposed to reduce the residual. However, a number of 6 specimens only are tested at variable strain rates. More tests are required to further confirm the strain rate effects. The plots of Figure 38 (c) and Figure 39(c) show that the residuals have a random behaviour and tend to a normal distribution, which indicate a satisfactory representativeness of the model.

(a)

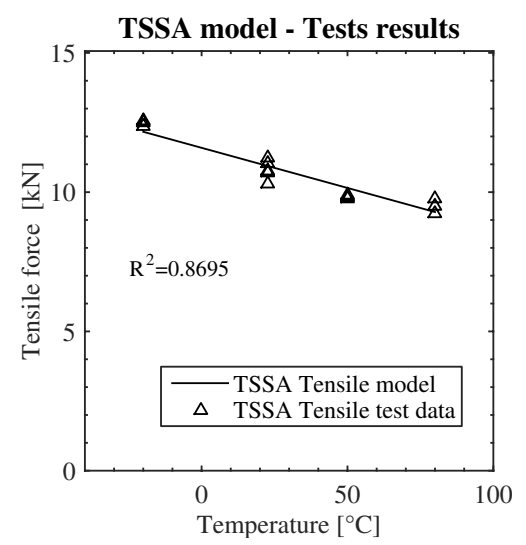

(b)

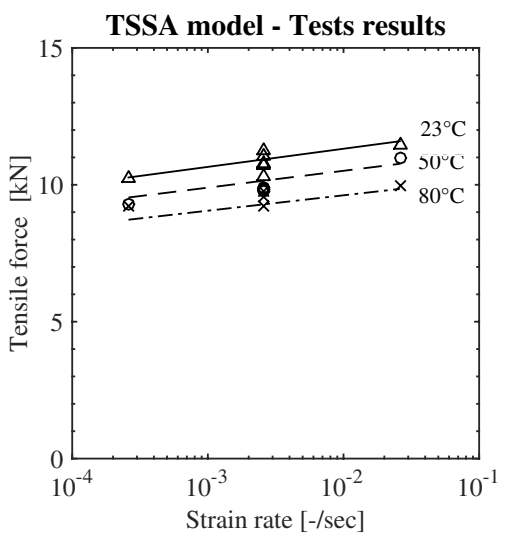

(c)

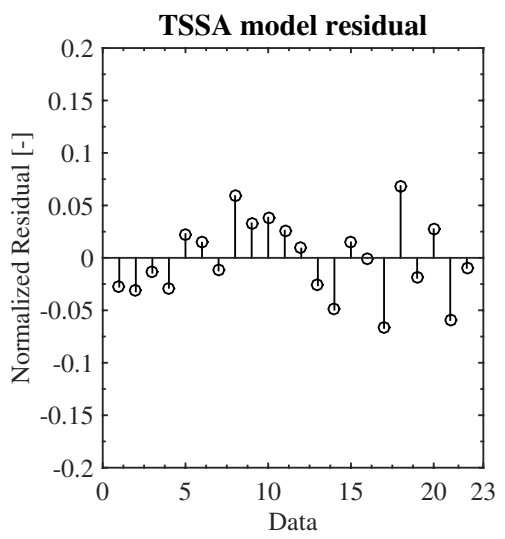

Figure 38: Comparison of the model with the experimental results versus (a) temperature for $1 \mathrm{~mm} / \mathrm{min}$ tests and (b) strain rate variations for $23^{\circ} \mathrm{C} 50$ and $80^{\circ} \mathrm{C}$ (continuous, dashed and dotted- dashed lines respectively) (c) normalized residual plot

(a)

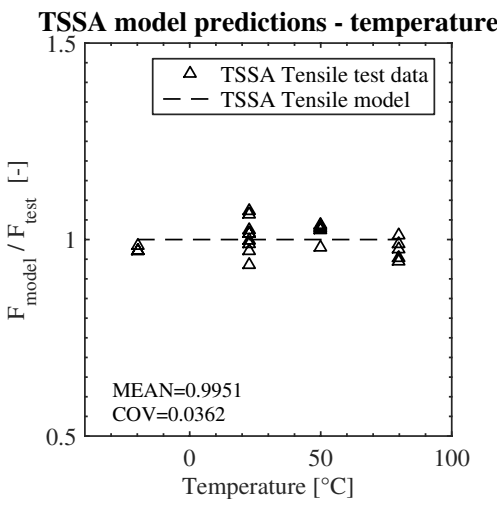

(b)

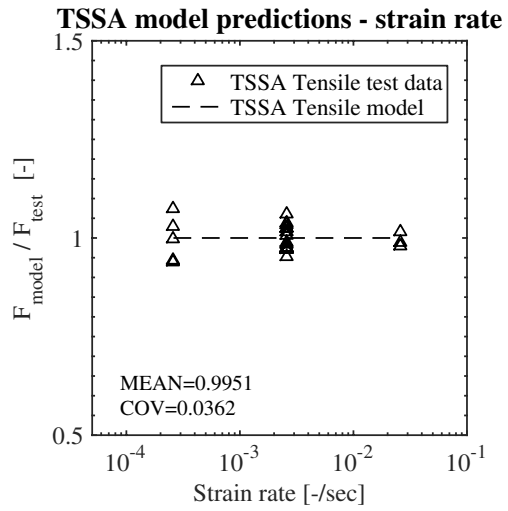

(c)

TSSA model residual

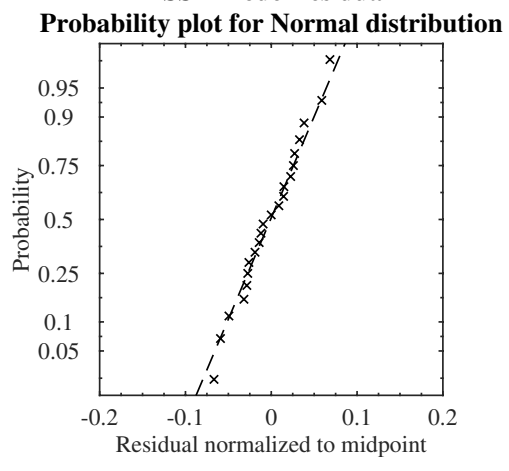

Figure 39: Model-test ratio for all the investigated configurations plotted versus (a) temperature (b) strain rate (c) Probability plot of the residual for normal distribution.

\subsection{Model for SG laminated connections under tensile load}

In this section the tensile resistances given by the SG model are compared with the experimental results. The residuals, calculated as the difference between model and experimental observations, are also plotted. The strain rate factor proposed for the SG model is given by equation (19). The temperature factor proposed for the SG model is given by equation (20). More specifically, a 
logarithmic law is proposed for the effects of strain rate variation, which varies with the square of the temperature. A mathematical expression based on inverse modified hyperbolic tangent function is instead proposed for the effects of temperature variation.

$$
\begin{gathered}
\alpha_{\varepsilon, S G}=2.62+\log (\dot{\varepsilon})\left(\left(\frac{13.46+T}{209.48}\right)^{2}+0.21\right) \\
\alpha_{T, S G}=0.27 \cdot\left(\tanh \left(\frac{61.06-T}{15.18}\right)+\tanh \left(\frac{13.46+T}{114.59}\right)+0.649\right)
\end{gathered}
$$

It is observed that the experimental results follow the proposed SG model for the full range of investigated temperatures, see Figure 40 (a) and Figure 41 (a). The experimental results also follow the proposed SG model for the strain rate variation, see Figure 40 (b) and Figure 41 (b). The deviation between model and test is small at room and high temperature. The residual are larger for the data at low temperature (see first five data of Figure 40 (c)). This is due to the large scatter of the experimental results at low temperature, as discussed in the experimental section of this work. The model appears then to be too conservative in predicting the strain rate effect at high temperature. The resistance measured at $80^{\circ} \mathrm{C}$ in the test is larger than the model (see outliers at $80^{\circ} \mathrm{C}$ of Figure 40 (b) and data 28 and 31 of Figure 41 (c)). A quadratic order equation for the strain rate coefficient might be evaluated to reduce the residual. However, a number of 6 specimens only are tested at variable strain rates. In that regard, more tests at different strain rates are required to further confirm the strain rate effect. The plot of Figure 40 (c) and Figure 41 (c) shows that the residuals have a random behaviour and tend to a normal distribution.

(a)

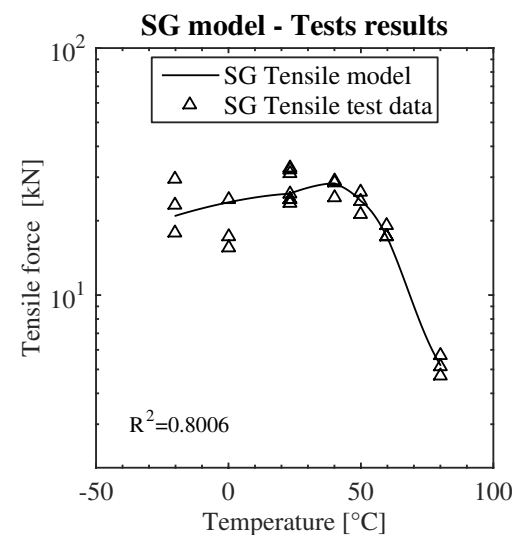

(b)

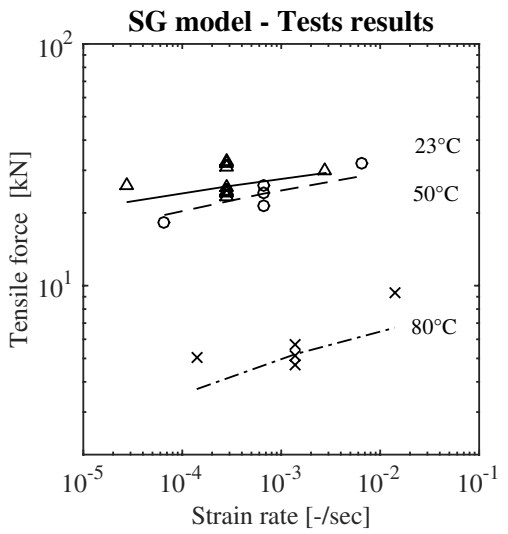

(c)

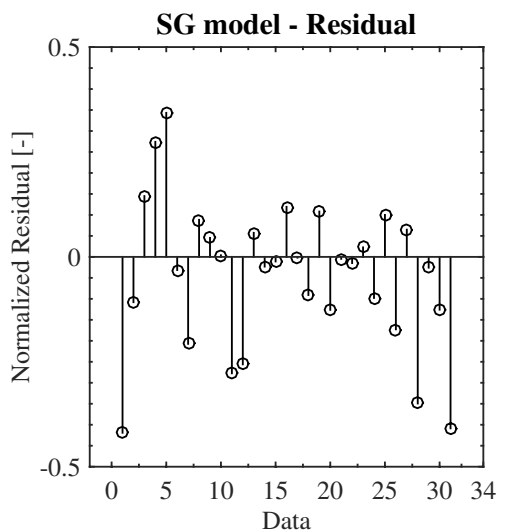

Figure 40: Comparison of the SG model with the experimental results versus (a) temperature for $1 \mathrm{~mm} / \mathrm{min}$ tests and (b) strain rate variations for $23^{\circ} \mathrm{C} 50$ and $80^{\circ} \mathrm{C}$ (continuous, dashed and dotted- dashed lines respectively) (c) normalized residual plot 
(a)

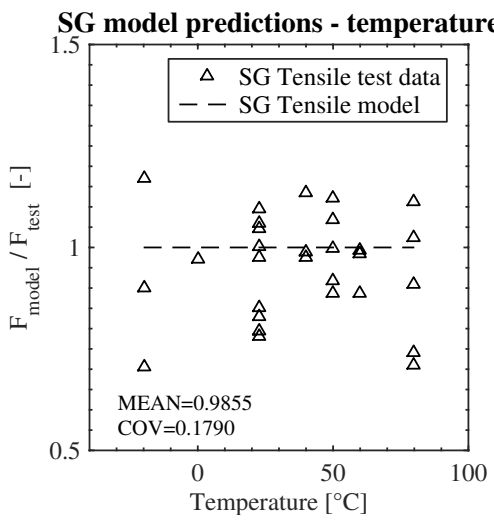

(b)

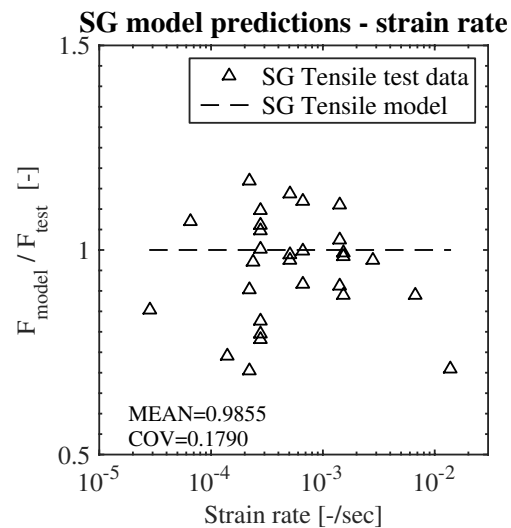

(c)

SG model residual Probability plot Normal distribution

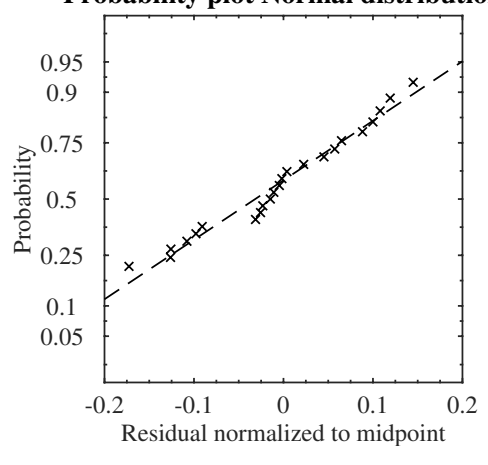

Figure 41: Ratio of model-test for all the investigated configurations plotted versus (a) temperature (b) strain rate (c) Probability plot of the residual for normal distribution. 


\section{Conclusions}

In this work the behaviour of laminated connections under tensile loading is studied by means of experimental, analytical and numerical analyses.

The experimental investigations showed that temperature and strain rate variations have important effects on the mechanical response of the connections. The resistance is proportional to the logarithm of the strain rate, and decreases at high temperature. At low temperature, the resistance increases for TSSA and slightly decreases for SG. Failure mode and failure location change as well with temperature for SG connections. Two main interesting phenomena are observed: the whitening phenomenon in TSSA and the bubble formation within the SG adhesive. These two phenomena suggest the hypothesis of having a dominant hydrostatic component of the stress tensor as the consequence of the large confinement state of the adhesive.

Analytical studies are carried out to investigate the mechanical response and the stress state in the adhesive. The results show that the confinement state induces a non-uniform stress distribution in the adhesive. In more detail, it is concluded that in the central part of the adhesive the application of longitudinal stress develops significant transversal stresses. This leads to a large hydrostatic component of the stress tensor, in agreement with the conclusions of the experimental study. The analytical expressions derived for the confinement effects are temperature dependent. Furthermore, it is also concluded that in case of rigid adhesives the glass deformability influences the stress field distribution in the adhesive. The hypothesis of rigid adherends leads to significant deviation from the actual connection behaviour and must not be assumed in numerical analysis.

Three-dimensional finite numerical analyses are performed to determine the adhesive stress state and the non-linear field distribution. Firstly, it is shown that the stress field deviates from the uniform distribution with a large gradient over the adhesive area. The output of the finite numerical model shows a stress state with a large hydrostatic component of the stress tensor. The model also gives a small transition region close to the adhesive perimeter with smaller triaxiality than in the central part. Secondly, the predictions of the numerical analyses are compared with the experimental observations. The different mechanical phenomena computed by the numerical models are confirmed by the observations of the experimental campaigns. Thirdly, the full set of numerical results is synthetized by the definition of so-called stress factors. The latters allow to derive the three-dimensional stress state in the adhesive at different temperatures and to compute the stress peak in the non-linear stress field distribution.

Finally, prediction models are proposed for the tensile resistance of TSSA and SG laminated connections. The models are obtained by developing a Matlab algorithm that allows the derivation of multi-dimensional non-linear models with variable standard deviations. It is observed that the models are able to compute the mechanical resistance of the laminated connections for the full range of investigated strain rates and temperatures. More specifically, a logarithmic law is proposed for the strain rate effects for both TSSA and SG connections. Linear and inverse hyperbolic-tangent-based laws are instead proposed for the TSSA and SG temperature effects, respectively.

\section{Acknowledgements}

The authors would like to thank the Swiss National Science Foundation for founding the present research (Grants 200020_150152 and 200021_134507). In addition, Glas Trösch AG Swisslamex, Kuraray (former Dupont), Dow Corning and Nuova Oxidal are gratefully acknowledged for the material support and specimens preparation. Finally, the COST Action TU0905 "Structural Glass Novel Design Methods and Next Generation Products" is also acknowledged for facilitating the research network. 


\section{References}

[1] L. Blandini, "Structural Use of Adhesive in Glass Shells," Thesis Dissertation, University of Stuttgart, 2005.

[2] J. Belis, A. Van Hulle, D. Callewaert, and J. Dispersyn, "Experimental investigation of unconventional canopy prototypes, suspended by adhesive bonds," in Challenging Glass 3, 2012.

[3] B. Weller, F. Nicklisch, V. Prautzsch, and I. Vogt, "Outline of Testing and Evaluation Program Used in Selection of Adhesives for Transparent Adhesive Joints in All-Glass Load-Bearing Structures," J. ASTM Int., vol. 9, no. 4, p. 104088, Apr. 2012.

[4] V. Dias, O. Hechler, and C. Odenbreit, "Determination of adhesives properties for non-linear numerical simulation of structural steel-glass connections," in Challenging Glass 3, 2012.

[5] M. Overend, S. Nhamoinesu, and J. Watson, "Structural Performance of Bolted Connections and Adhesively Bonded Joints in Glass Structures," J. Struct. Eng., vol. 139, no. 12, 2013.

[6] M. Overend, Q. Jin, and J. Watson, "The selection and performance of adhesives for a steelglass connection," Int. J. Adhes. Adhes., vol. 31, no. 7, pp. 587-597, Jun. 2011.

[7] E. M. P. Huveners, F. Van Herwijnen, F. Soetens, and H. Hofmeyer, "Mechanical shear properties of adhesives," in Glass Performance Days, 2007.

[8] I. Vogt, "Strukturelle Klebungen mit UV- und lichthärtenden Acrylaten," Thesis Dissertation, Technical University of Dresen, 2009.

[9] J. Schneider and J. Franz, "Classification of the material properties of interlayers for laminated glass regarding the post-breakage behavior," in Engineered Transparency, 2012.

[10] V. A. Silvestru and O. Englhardt, "Application study for hybrid adhesively bonded glass-steel façade elements," in Challenging Glass 4 \& COST Action TU0905 Final Conference, 2014.

[11] S. Marinitsch and C. Schranz, "Folded plate structures made of glass laminates : a proposal for the structural assessment," Glas. Struct. Eng., 2015.

[12] J. Dispersyn, J. Belis, and D. Sonck, "New glass design method for adhesive point-fixing applications," Proc. ICE - Struct. Build., 2015.

[13] J. O'Callaghan, “Thinking big with structural glass," in Glass Performance Days, 2009.

[14] J. O'Callaghan, “Adventures with Structural Glass,” in Glass Performance Days, 2012.

[15] P. Lenk and H. Lambert, "Challenges in the design, fabrication and installation of glass structures comprising of super jumbo glass sheets," in Challenging Glass 3, 2012.

[16] P. Lenk and F. Lancaster, "Connections in structural glass," in Glass Performance Days, 2013.

[17] B. Kassnel-Henneberg, "Connections in Glass," in Challenging Glass 5, 2016.

[18] S. Sitte and A. Wolf, "TSSA-bonded point-supported structural glazing of insulating glass units at Dow Corning's European Distribution Center," in Engineered Transparency, 2012.

[19] J. Ludwig, "Tottenham Court Road Station - Plaza entrances structures and building structural glass," in Challenging Glass 4 \& COST Action TU0905 Final Conference, 2014.

[20] J. Watson, "Novel Structural Glass Connections for Architectural Applications," Thesis Dissertation, University of Cambridge, 2013.

[21] S. Sitte, M. J. Brasseur, L. D. Carbary, and A. T. Wolf, "Preliminary Evaluation of the Mechanical Properties and Durability of Transparent Structural Silicone Adhesive ( TSSA ) for Point Fixing in Glazing," J. ASTM Int., vol. 8, no. 10, pp. 1-27, 2011.

[22] S. Sitte, "Experimental evaluation of mechanical properties of TSSA," 2011.

[23] A. T. Wolf and K. Hirai, "Evaluation of transparent structural silicone film adhesive for 
bonded point-fixed glazing," in Engineered Transparency, 2010, pp. 167-177.

[24] P. Vandereecken, M. Elliott, and M. Plettau, "Durable high design strength in glass curtain wall," in Challenging Glass 4 \& COST Action TU0905 Final Conference, 2014, pp. 401-408.

[25] A. Hagl, O. Dieterich, A. T. Wolf, and S. Sitte, "Tensile Loading of Silicone Point Supports Revisited," in Challenging Glass 3, 2012.

[26] A. Hagl, A. T. Wolf, and S. Sitte, "Investigation of Stress-Whitening in Transparent Structural Silicon Adhesive," in Challenging Glass 3, 2012.

[27] R. Decourcelle and F. Levasseur, "Long term behaviour of laminated glass plates by bending creep experiments," in ISAAG, 2010.

[28] D. Delincé, "Experimental Approaches for Assessing Time and Temperature Dependent Performances of Fractured Laminated Safety Glass," Thesis Dissertation, Ghent University, 2014.

[29] I. Stelzer, "High Performance Laminated Glass," in Challenging Glass 2, 2010.

[30] I. Calderone, "Effective Laminate Thickness for the Design of Laminated Glass," 2009.

[31] S. J. Bennison and P. S. Davies, "High-performance laminated glass for structurally efficient glazing," in Innovative light-weight structures and sustainable façades, 2008.

[32] J. Belis, J. Vander Beken, R. Van Impe, and D. Callewaert, "Performance of glass-ionoplast laminates above room temperature," in Glass Performance Days, 2007.

[33] D. Callewaert, J. Belis, D. Delincé, and R. Van Impe, "Experimental stiffness characterisation of glass/ionomer laminates for structural applications," Constr. Build. Mater., vol. 37, pp. 685692, 2012.

[34] J. Belis, C. Bedon, C. Louter, C. Amadio, and R. Van Impe, "Experimental and analytical assessment of lateral torsional buckling of laminated glass beams," Eng. Struct., vol. 51, pp. 295-305, 2013.

[35] C. Bedon, J. Belis, and A. Luible, "Assessment of existing analytical models for the lateral torsional buckling analysis of PVB and SG laminated glass beams via viscoelastic simulations and experiments," Eng. Struct., vol. 60, pp. 52-67, 2014.

[36] M. Santarsiero, C. Louter, and A. Nussbaumer, "Laminated connections for structural glass components : a full-scale experimental study," Glas. Struct. Eng., 2016.

[37] J. Schneider, S. Kolling, J. Kuntsche, and S. Moennich, "Tensile properties of different polymer interlayers under high strain rates," in Engineered Transparency, 2012.

[38] K. Puller, J. Denonville, and W. Sobek, "An innovative glass connection technique using an ionomer interlayer," in Glass Performance Days, 2011.

[39] M. Meissner and V. Sackmann, "On the Effect of Artificial Weathering on the Shear Bond and the Tear Strength of two different Interlayers of Laminated Glass," in ISAAG, 2006.

[40] J. Kuntsche and J. Schneider, "Mechanical behaviour of polymer interlayers in explosion resistant glazing," in Challenging Glass 4 \& COST Action TU0905 Final Conference, 2014, pp. $447-454$.

[41] L. Biolzi, S. Cattaneo, and G. Rosati, "Progressive damage and fracture of laminated glass beams," Constr. Build. Mater., vol. 24, no. 4, pp. 577-584, 2010.

[42] S. J. Bennison, J. G. Sloan, D. F. Kristunas, P. J. Buehler, T. Amos, and C. A. Smith, "Laminated Glass for Blast Mitigation: Role of Interlayer Properties," in Glass Processing Days, 2005.

[43] J. Belis, J. Depauw, D. Callewaert, D. Delincé, and R. Van Impe, "Failure mechanisms and residual capacity of annealed glass/SGP laminated beams at room temperature," Eng. Fail. Anal., vol. 16, no. 6, pp. 1866-1875, 2009.

[44] M. Santarsiero, C. Louter, and A. Nussbaumer, "The mechanical behaviour of SentryGlas 
ionomer and TSSA silicon bulk materials at different temperatures and strain rates under uniaxial tensile stress state," Glas. Struct. Eng., 2016.

[45] S. Peters, A. Fuchs, J. Knippers, and S. Behling, "Ganzglastreppe mit transparenten SGPKlebeverbindungen - Konstruktion und statische Berechnung," Stahlbau, vol. 76, no. 3, pp. 151-156, Mar. 2007.

[46] J. Belis, A. Van Hulle, B. Out, F. Bos, D. Callewaert, and H. Poulis, "Broad screening of adhesives for glass-metal bonds," in Glass Performance Days, 2011, pp. 286-289.

[47] A. Van Hulle, J. Belis, D. Callewaert, L. Scheerlinck, and B. Out, "Development of structural adhesive point-fixings," in Glass Performance Days, 2011.

[48] J. Belis, D. Callewaert, and A. Van Hulle, Bouwen met glas en adhesieven - praktische gids voor ontwerper en uitvoerder. Universiteit Gent, Laboratorium voor modelonderzoek, 2012.

[49] K. Puller, "Untersuchung des Tragverhaltens von in die Zwischenschicht von Verbundglas integrierten Lasteinleitungselementen," Thesis Dissertation, University of Stuttgart, 2012.

[50] P. Carvalho and P. J. S. Cruz, "Connecting Through Reinforcement - Experimental Analysis of A Glass Connection Using Perforated Steel Plates," in Challenging Glass 3, 2012.

[51] M. Santarsiero, P. Carvalho, C. Louter, and P. J. S. Cruz, "Experimental and numerical investigations of metal-to-glass embedded connections with thin stainless steel plate," in COST Action TU0905, Mid-term Conference on Structural Glass, 2013.

[52] J. Ludwig, "Tottenham Court Road Station Upgrade - Structural Glass Plazas," in International Conference on Building Envelope Design and Technology, 2015.

[53] Kuraray and obtained through web site http://glasslaminatingsolutions.kuraray.com, "SentryGlas ${ }^{\circledR}$ - data sheet." 2009.

[54] Dow Corning and obtained through website www.dowcorning.com, "TSSA - Transparent Structural Silicone Adhesive - data sheet." 2013.

[55] CEN - European Committee for Standardisation, "EN 572 - 1 - Glass in building - Basic soda lime silicate glass products - Part 1: Definitions and general physical and mechanical properties." 2004.

[56] CEN - European Committee for Standardisation, "EN 572 - 2 - Glass in building - Basic soda lime silicate glass products - Part 2: Float glass."

[57] M. Haldimann, A. Luible, and M. Overend, Structural Use of Glass. International Association for Bridge and Structural Engineering - IABSE, 2008.

[58] CEN - European Committee for Standardisation, "Eurocode 3: Design of steel structures - Part $1-4$ : General rules - Supplementary rules for stainless steels.".

[59] CEN - European Committee for Standardisation, "EN 10088-2 - Stainless steel - Part 2: Technical delivery conditions for sheet/plate and strip of corrosion resisting steels for general purposes.".

[60] R. Decourcelle, J. C. Nugue, and F. Levasseur, "Mechanical participation of interlayer on laminated glass for building applications," in Glass Performance Days, 2009, pp. 718-721.

[61] L. Calgeer, "Structural design of glass façade components: comparison of different connection typologies," Master Thesis Dissertation, École Polytechnique Fédérale De Lausanne - EPFL, 2015.

[62] European Organisation for Technical Approvals, "ETAG 002 - Guideline for European Technical approval for Structural Sealant Glazing Systems ( SSGS ) Part 1: Supported and unsupported system.” 2001.

[63] S. Toki, T. Fujimaki, and M. Okuyama, "Strain-induced crystallization of natural rubber as detected real-time by wide- angle X-ray diffraction technique," Polymer (Guildf)., vol. 41, no. 14, pp. 5423-5429, 2000.

[64] A. Heaton, The chemical industry. Blackie \& Son Limited. 
[65] Ehrenstein, Polymeric Materials: Structure, Properties, Applications. Hanser, 2001.

[66] D. François, A. Pineau, and A. Zaoui, Mechanical Behaviour of Materials - Volume I: Microand Macroscopic Constitutive Behaviour. Springer, 2012.

[67] D. François, A. Pineau, and A. Zaoui, Mechanical behaviour of Materials - Volume II: Fracture Mechanics and Damage. Springer, 2013.

[68] G. Márkus, Kreis- und Kreisringplatten unter periodischer Belastung. Werner-Verlag, 1985.

[69] G. Márkus, Theorie und Berechnung rotationssymmetrischer Bauwerke. Werner-Verlag, 1967.

[70] M. Santarsiero, "Laminated connections for structural glass applications," Thesis Dissertation, École Polytechnique Fédérale De Lausanne - EPFL, 2015.

[71] SIMULIA, Abaqus Analysis User's Manual - Volume II: Analysis, vol. II. 2011.

[72] SIMULIA, Abaqus Analysis User's Manual - Volume IV: Elements, vol. IV. 2011. 


\section{Appendix A - Full set of tensile test results}

\section{SG tensile test results}
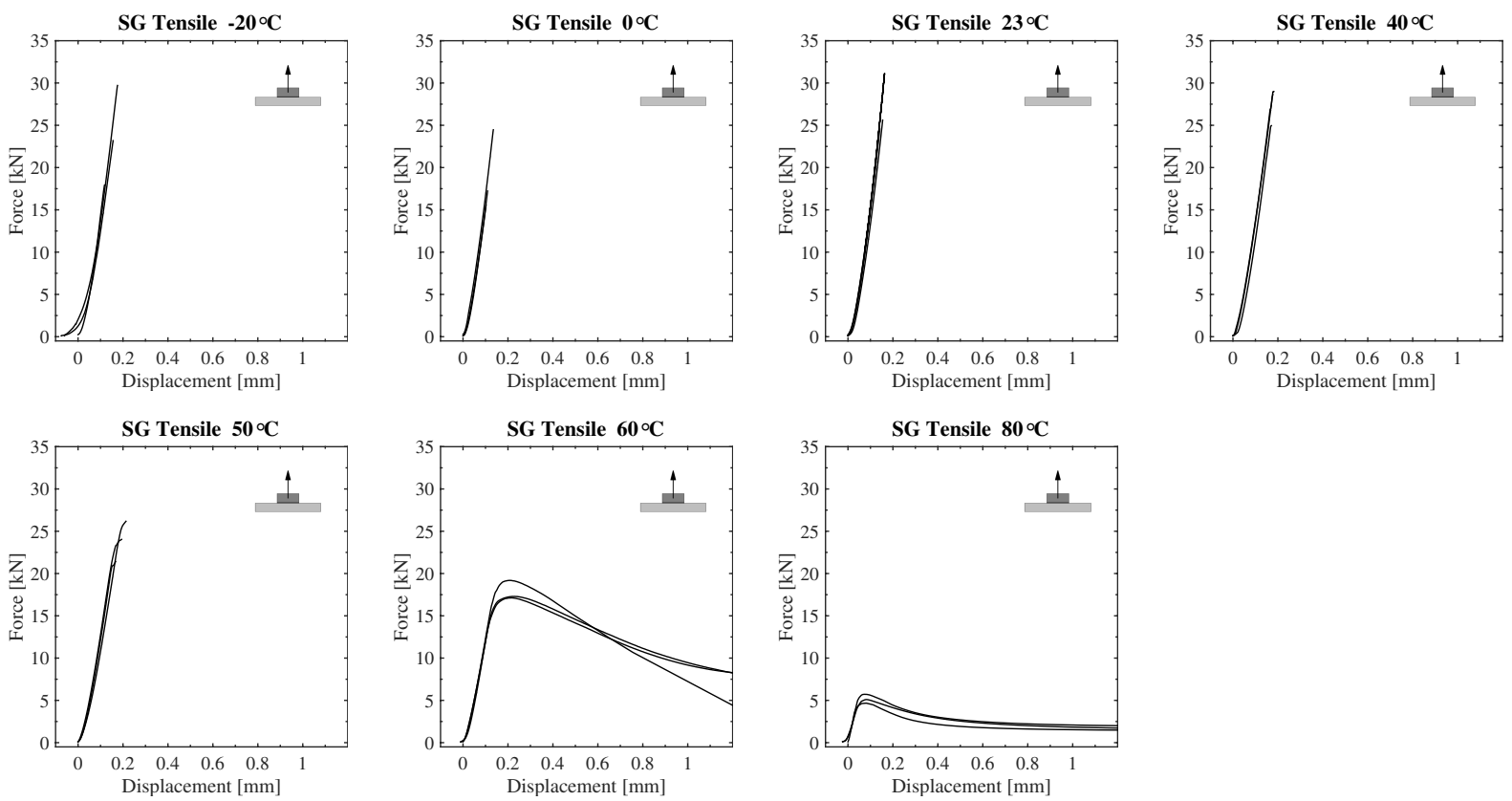

TSSA tensile test results
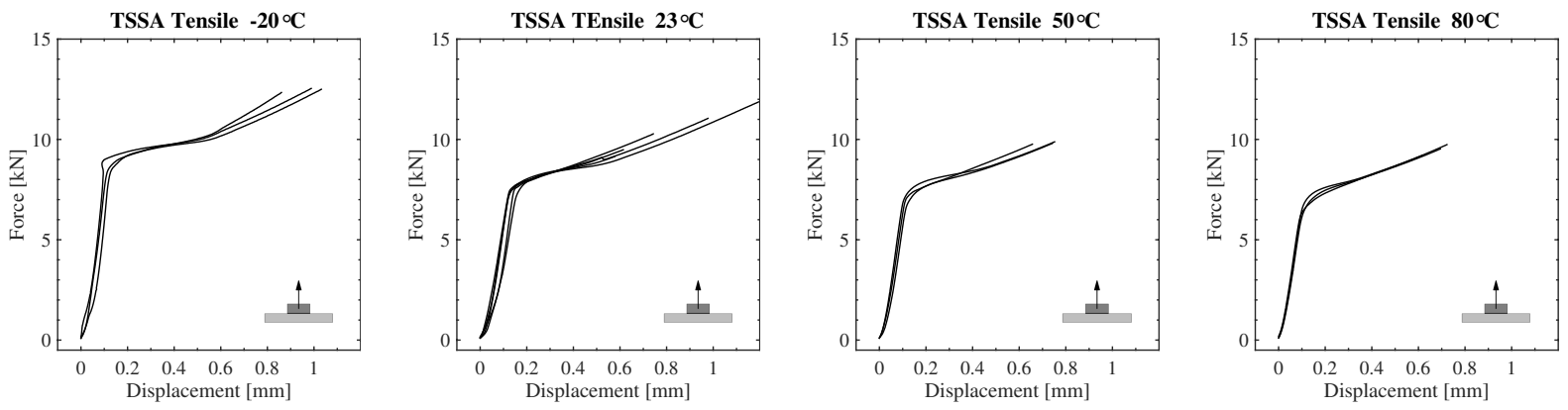